
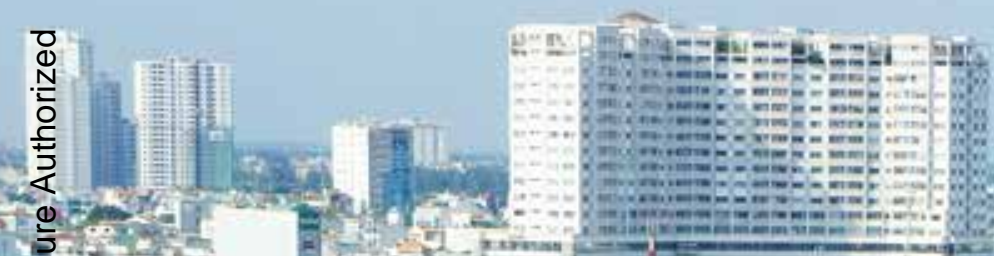

W.

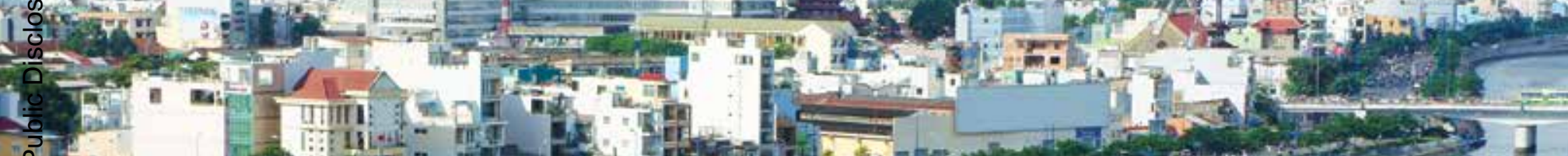
$\frac{0}{5}+\frac{1}{2}$

a - I:

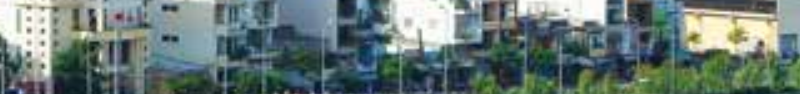

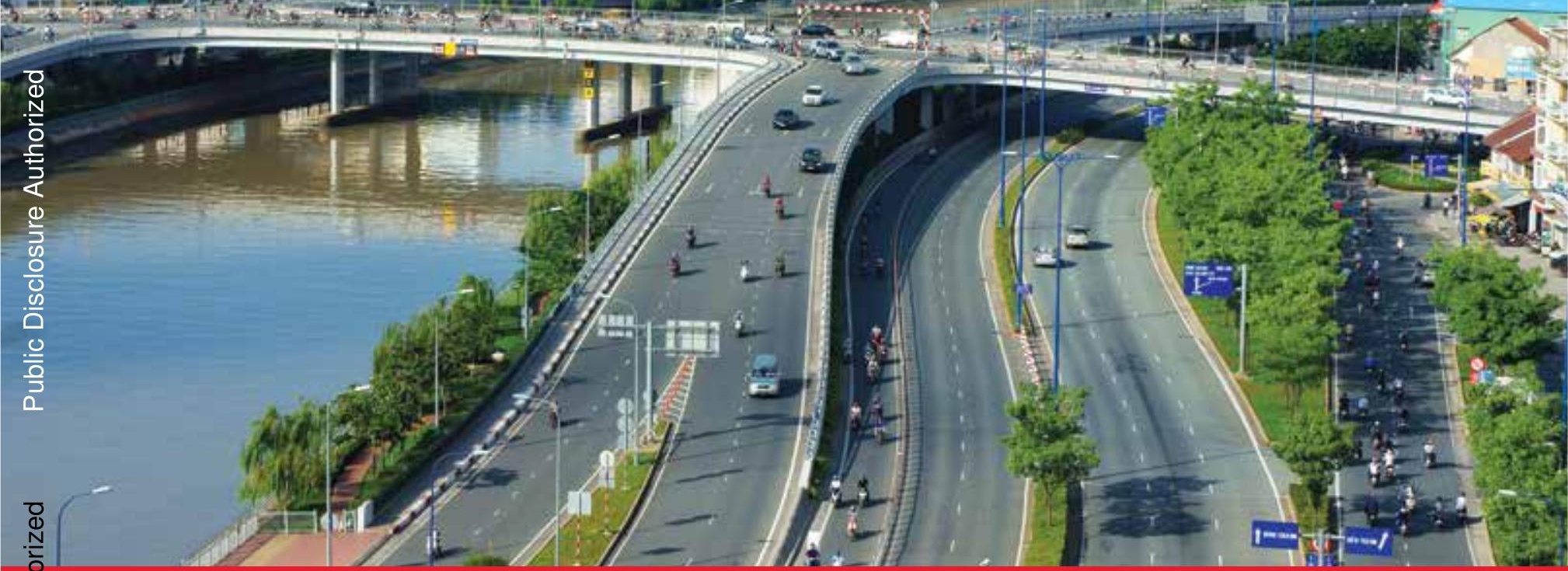

AUSTRALIA-WORLD BANK GROUP STRATEGIC PARTNERSHIP IN VIETNAM Public Investment Management

\title{
PUBLIC INVESTMENT MANAGEMENT IN VIETNAM
}

Assessment and Reform Priorities for Overcoming the Bottlenecks 



\title{
PUBLIC INVESTMENT MANAGEMENT IN VIETNAM
}

\author{
Assessment and Reform Priorities for \\ Overcoming the Bottlenecks
}

The World Bank

July 2018 


\section{TABLE OF CONTENTS}

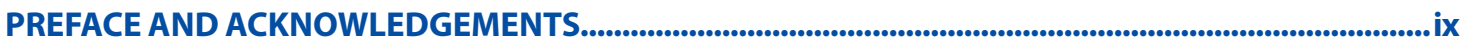

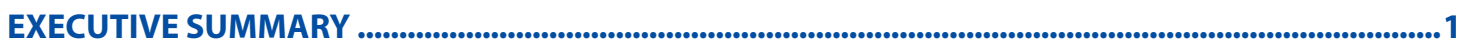

1. INTRODUCTION: CONTEXT, RATIONALE AND METHODS ......................................................................9

2. CAPITAL SPENDING TRENDS AND PATTERNS..............................................................................................17

Capital Spending Financed by the State Budget..............................................................................................17

Decentralization of Capital Spending Mandates and Authorities ..............................................................2

Government Bond Financing.....................................................................................................................................

3. AN INSTITUTIONAL MAPPING OF PIM IN VIETNAM...........................................................................23

Institutional Arrangements and Coordination in PIM .................................................................................2

Important Changes in Upstream Part of Project Cycle..........................................................................................2

Recent Changes with regards to ODA Projects .....................................................................................................2

4. STRATEGIC GUIDANCE AND FIRST-LEVEL SCREENING ....................................................................33

What Does Good Practice Look Like? .........................................................................................................................

Assessment of Strategic Guidance for Public Investment in Vietnam...........................................................33

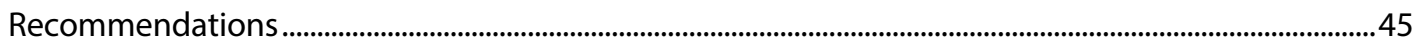

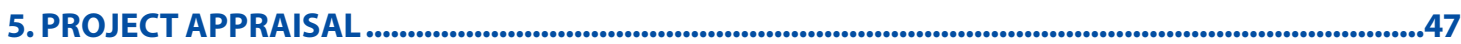

What Does Good Practice Look Like? .........................................................................................................................4

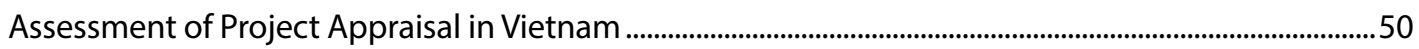

Recommendations ..................................................................................................................................................62

6. INDEPENDENT REVIEW OF PROJECT APPRAISAL ..........................................................................................63

What Does Good Practice Look Like? ............................................................................................................................63

Institutional Arrangements for Independent Review: ....................................................................................64

Assessment of Independent Review in Vietnam ..................................................................................................66

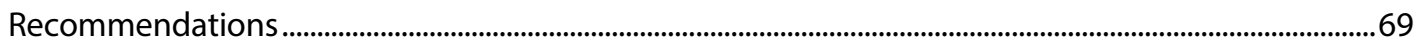

7. SELECTION AND BUDGETING ..............................................................................................................................71

What Does Good Practice Look Like? ........................................................................................................................71

Assessment of Selection and Budgeting in Vietnam .....................................................................................73

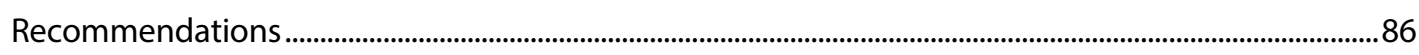

8. PROJECT IMPLEMENTATION...................................................................................................................89

What Does Good Practice Look Like? ........................................................................................................................89

Assessment of Project Implementation in Vietnam ..........................................................................................91

Recommendations ................................................................................................................................................. 102 


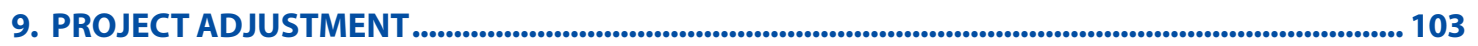

What Does Good Practice Look Like? .................................................................................................................................. 103

Assessment of Monitoring and Project Adjustment in Vietnam ................................................................ 105

Recommendations .................................................................................................................................................. 112

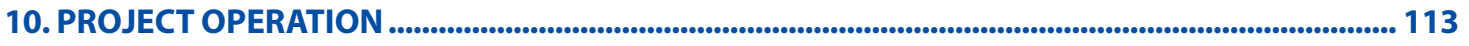

What Does Good Practice Look Like? ....................................................................................................................... 113

Assessment of Project Operation in Vietnam.................................................................................................... 114

Recommendations .................................................................................................................................................. 117

11. PROJECT EVALUATION .................................................................................................................................. 119

What Does Good Practice Look Like? ............................................................................................................................. 119

Assessment of Project Ex Post Evaluation in Vietnam ....................................................................................... 120

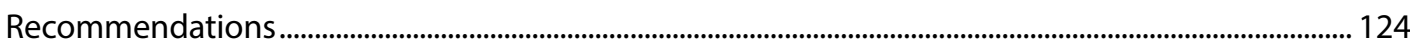

12. FINDINGS AND POLICY RECOMMENDATIONS ................................................................................. 125

Key Constraints on PIM System Functioning ................................................................................................. 125

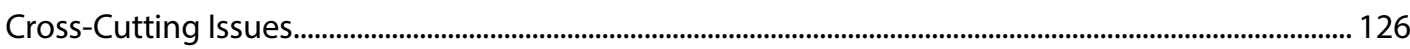

Summary of Recommendations by PIM Function .......................................................................................... 127

ANNEX 1: SUMMARY OF FINDINGS FROM THE SURVEY OF TRANSPORT PROJECTS ................... 131

ANNEX 2: SUMMARY OF FINDINGS FROM THE SURVEY OF EDUCATION PROJECTS....................... 134

\section{FIGURES}

Figure A.1: WBG's PIM Systemic Framework: Eight Must-Have Functions ......................................................... 2

Figure 1: State budget as share of total investment and GDP, 2006-15 .....................................................17

Figure 2: Public investment in East Asia Pacific and OECD countries (percent of GDP), 2004-2011 .....17

Figure 3: Difference between budgeted and actual capital spending (percent), 2006-15 ...................18

Figure 4: Sub-sectoral breakdown of capital spending in transport and agriculture sectors,

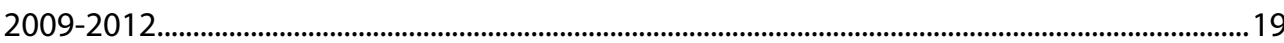

Figure 5: Core Functions of Public Investment Management in Vietnam ...................................................24

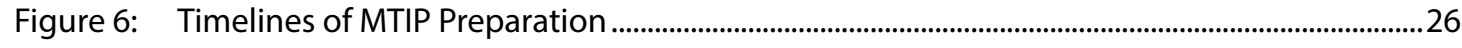

Figure 7: Appraisal and Approval of Investment Intention Report..............................................................28

Figure 8: Steps in ODA Project Concept Idea.......................................................................................................30

Figure 9: A Strong Appraisal Process Reduces the Risk of Costly Project Adjustments during Implementation ..............................................................................................................................4

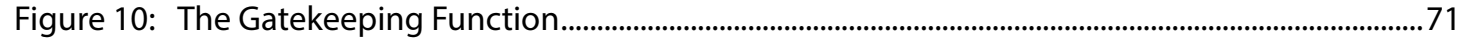

Figure 11: Capital Baseline and Fiscal Space for Public Investment Projects................................................82 


\section{TABLES}

Table 1: Sample of Projects from the Transport Sector... 14

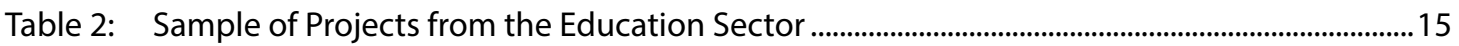

Table 3: Funding sources of public investment (percent), 2001-15 .............................................................. 18

Table 4: $\quad$ Budget capital spending by sector (VND billion), 2000-14 .............................................................. 19

Table 5: Capital spending by tier of government and sector (VND billion), 2000-14 .............................20

Table 6: Allocation of Responsibilities for Appraisal and Decision-Making in the PIL ...........................50

Table 7: $\quad$ Proportionality in the Application of Assessment Tools in Ireland ...............................................56

Table 8: Reponses to Questions on Climate Change Resilience from Sample Survey of

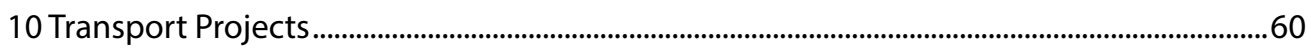

Table 9: Adequacy of Budget Funding for Sampled Transport Projects .....................................................8 80

Table 10: Summary Findings on Project Management and Implementation Planning for Transport Projects in the Project Sample

Table 11: Summary Findings on Project Management and Implementation Planning for Education rojects in the Project Sample

Table 12: Red-Amber-Green Delivery Confidence Rating Used in the United Kingdom and New Zealand. 110

\section{BOXES}

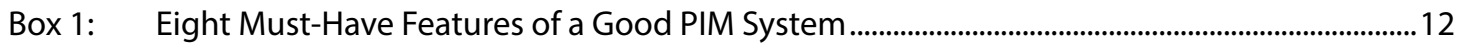

Box 2: $\quad$ Planning and Budgeting for Capital Investment at Local Level.....................................................36

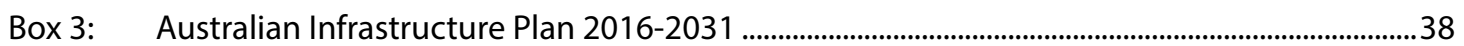

Box 4: $\quad$ Examples of Formats for Project Profiles .............................................................................................4

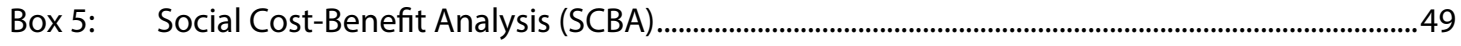

Box 6: $\quad$ Main Differences between Financial and Economic Analysis......................................................52

Box 7: $\quad$ Chile's Sector Guidelines on Analytical Methods .............................................................................54

Box 8: $\quad$ The Netherlands' General Guidance for Social Cost-Benefit Analysis ............................................55

Box 9: $\quad$ Examples of Demand Shortfalls for European Transport Infrastructure Projects.....................59

Box 10: The Problems of Optimism Bias in Projects...................................................................................63

Box 11: Questions for Independent Reviewers in France ...............................................................................64

Box 12: Country Differences in the Depth of Independent Review ...........................................................65

Box 13: Country Variations in Outcome of Independent Review .............................................................66

Box 14: Linking Strategic Planning, Project Appraisal and Budgeting in the Netherlands ..................75

Box 15: Limitations of Economic Analysis Methods for Selection and Budgeting ..................................76

Box 16: Capital Budgeting in a Medium-Term Perspective in Ireland .......................................................78

Box 17: Metrolink Project Management Manual - Table of Contents .........................................................94 
Box 18: South Africa's Land Aquisition Process ..........................................................................................99

Box 19: Columbia's Unified System for Public Finance and Investment (SUIFP)......................................99

Box 20: Re-assessment and Adjustment in South Korea ...................................................................... 105

Box 21: Ireland's Guidelines on Project Adjustment.................................................................................. 108

Box 22: South Korea's Total Project Cost Management System............................................................... 109

Box 23: Indicative Budgeting Guidelines for Various Types of Infrastructure........................................ 116

Box 24: Ireland's Approach to Ex Post Evaluation ....................................................................................... 119

Box 25: Format for a Project Completion Report ................................................................................... 122

Box 26: Qualitative and Quantitative Evaluation Criteria ....................................................................... 123 


\section{ACRONYMS AND ABBREVIATIONS}

\begin{tabular}{|c|c|}
\hline CEA & Cost-Effectiveness Analysis \\
\hline $\mathrm{CL}$ & Construction Law \\
\hline EIRR & Economic Internal Rate of Return \\
\hline FDI & Foreign Direct Investment \\
\hline FIRR & Financial Internal Rate of Return \\
\hline FS & Feasibility Study \\
\hline$M \& E$ & Monitoring and Evaluation \\
\hline MCA & Multi-Criteria Analysis \\
\hline MOC & Ministry of Construction \\
\hline MOET & Ministry of Education and Training \\
\hline MONRE & Ministry of Natural Resources and Environment \\
\hline MOF & Ministry of Finance \\
\hline MOT & Ministry of Transport \\
\hline MPI & Ministry of Planning and Investment \\
\hline MTBF & Medium-Term Budget Framework \\
\hline MTEF & Medium-Term Expenditure Framework \\
\hline MTFF & Medium-Term Fiscal Framework \\
\hline MTIP & Medium-Term Investment Plan \\
\hline MTPF & Medium-Term Performance Framework \\
\hline NPV & Net Present Value \\
\hline O\&M & Operations and Maintenance \\
\hline OOG & Office of Government \\
\hline PFS & Pre-Feasibility Study \\
\hline PIL & Public Investment Law \\
\hline PIM & Project Investment Management \\
\hline PIU & Project Implementation Unit \\
\hline PMU & Project Management Unit \\
\hline ROII & Report of Investment Intention \\
\hline SCBA & Social Cost-Benefit Analysis \\
\hline SEDP & Socio-Economic Development Plan \\
\hline SDR & Social Discount Rate \\
\hline SEDS & Socio-Economic Development Strategy \\
\hline SBL & State Budget Law \\
\hline SBV & State Bank of Vietnam \\
\hline TPCM & Total Project Cost Management \\
\hline WBG & World Bank Group \\
\hline
\end{tabular}




\section{PREFACE AND ACKNOWLEDGEMENTS}

The report "Public Investment Management in Vietnam: Assessment and Reform Priorities for Overcoming the Bottlenecks" was prepared by Simon Groom, Quang Hong Doan, and Phuong Anh Nguyen under the guidance of Ousmane Dione (Country Director), Deepak Mishra and Robert Taliercio (Practice Managers).

The report was a part of a World Bank technical assistance program which undertook a diagnostic assessment of Vietnam's public investment management system in order to identify weaknesses compared to good practice models, determine priorities for reform and make specific recommendations on actions over the short, medium and long term.

The authors are grateful to Jay Hyung Kim for sharing invaluable ideas and practical experiences as well as providing technical advices during the technical assistance and the preparation of the report. Contribution of Dinh Trong Thang (Central Institute for Economic Management) in the technical assistance, especially in leading the field works for the collection of project information is greatly appreciated. We gratefully acknowledge insightful comments and suggestions from Achim Fock, Nicola Smithers, Sebastian Eckardt, Son Duy Nguyen, Tuan Minh Le, Arvind Nair, and participants from Ministry of Planning and Investment, Ministry of Finance, Ministry of Transport, Ministry of Construction, Ministry of Education and Training, and from several provinces in the consultation workshop held in collaboration with the Ministry of Planning and Investment.

Financial support from the Second Australia - World Bank Strategic Partnership in Vietnam (ABP-2), an Australian Trust Fund managed by the World Bank, for the consultation workshops and printing of the report is greatly appreciated. 



\section{Executive Summary}

\section{Rationale and Diagnostic Methods}

Vietnam's on-going reform program places emphasis on improving the efficiency of public investment in order to boost the country's economic performance, which remains highly dependent on factor accumulation. Investment of the state sector has been the most important source of capital accumulation in Vietnam, despite rapid growth of the domestic private sector and foreign direct investment (FDI) since the start of Doi Moi, the economic reform, in the late 1980s.

The urgency of reform is a result of the growing concern regarding the rapidly declining efficiency of public investment. The allocation of capital investment lacks strategic prioritization and is spreads too thinly in many areas. The completion rate of projects managed by central government was only 20 percent in 2000-14. Total state budget allocations for 2013-2015 could only accommodate less than half of total investment needs for approved projects from both central and local governments. Further evidence of inefficiency comes from the high level of capital expenditure arrears, which remains relatively unchanged even during the recent period of investment consolidation.

Despite the improvements introduced by the Government in recent years, including the issuance of the Public Investment Law, progress in restructuring of public investment has been slow and mostly focuses on cleaning up of the existing fragmented portfolio. Many measures are short-term in nature and aim to deal quickly with consequences rather than tackle the root causes of the problems. There is also no evidence-based, systemic and comprehensive blueprint for improving efficiency of public investment over the long term. A major factor behind the slow progress of restructuring public investment is the lack of a well-designed reform program with clear specific objectives and priorities.

Against this background, the Ministry of Planning and Investment (MPI) requested the World Bank to undertake a diagnostic assessment of Vietnam's public investment management (PIM) system in order to identify weaknesses compared to good practice models, determine priorities for reform and make specific recommendations on actions over the short, medium and long term.

The Report "Public Investment Management in Vietnam: Assessment and Reform Priorities for Overcoming Bottlenecks" has been prepared following the World Bank Group's PIM Diagnostic Framework as formalized in the 2014 publication, 'The Power of Public Investment'. This framework analyzes the presence and quality of institutional arrangements required to support the performance of eight "must-have" functionalities across the project and capital budgeting cycles (see Figure A1), thus enabling a robust assessment against good international practice. The framework yields a qualitative assessment of a country's PIM system, rather than an indicator-based rating. 
Figure A.1: WBG's PIM Systemic Framework: Eight Must-Have Functions

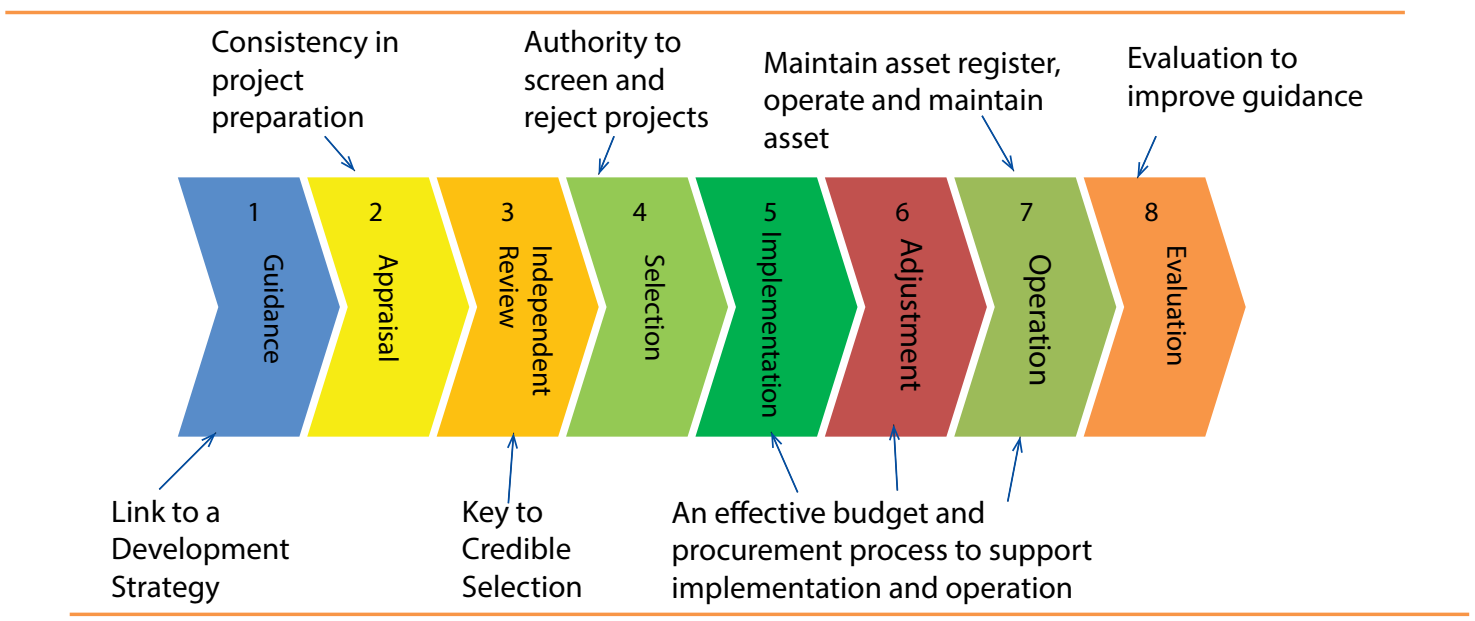

Source: The Power of Public Investment Management: Transforming Resources into Assets for Growth (World Bank, 2014).

To complement the interviews and written documentation on regulations and procedures, a project-level evidence base has been assembled by sampling 17 project case studies of on-going or completed public investment projects in the Transport and Education sectors ( 10 and 7 case projects, respectively). To assist in analyzing the selected projects in a consistent way, a detailed questionnaire was developed based around the World Bank's diagnostic framework. The findings of the case studies are brought into the analysis of the current PIM system.

\section{Main Findings and Headline Recommendations}

The efficiency of public investment has been identified as an important driver to future economic development in Vietnam, where growth will increasingly depend on getting more out of given resources, and less on factor accumulation. Towards this goal, Vietnam has recently made commendable efforts to strengthen the PIM system, including notable changes to improve the regulatory framework. The State Budget Law (SBL) and the Public Investment Law (PIL) have introduced medium-term planning and budgeting in an effort to integrate capital and recurrent budgeting and improve the alignment of the capital budget to development priorities. In addition, the PIL added an important first stage in the PIM system, whereby a formal decision is now taken on whether to proceed to a feasibility study and appraisal, on the basis of an early analysis of the project concept. The requirement for appraisal on the basis of a feasibility study and the practice of independent review of the resulting investment reports (introduced by the amended Construction Law) now aligns Vietnam with many aspects of recognised good practice.

Notwithstanding these positive developments in PIM, the effective implementation of these critical changes remains challenging. Improving public investment efficiency has to address the following existing areas of weakness: i) allocation of financial resources to weak, low priority and/or unaffordable projects; ii) failure to optimize the use of resources for investment once allocated, leading to cost overruns, delays and departures from specification; and iii) under-provision of complementary resources required to achieve service delivery targets for the assets created. The cumulative effects of earlier inefficiencies 
also lead to blockages, such as over-extended investment programs and arrears, which undermine wellintentioned reforms, even if these make sense in isolation.

More specifically, in relation to the eight key functionalities embodied in the World Bank PIM assessment framework, the main issues are:

(i) Vietnam has a large number of strategic documents but they lack the coherence and realism necessary to provide strategic guidance for public investment. First-level screening also has a narrow scope.

(ii) The practical status of project appraisal in the broader system remains ambiguous, and elements of good practice in project appraisal have not yet been fully operationalized.

(iii) The necessity for some form of independent review of project proposals is recognized in the legislation, but the design falls short of a fully impartial review of the feasibility study and appraisal findings.

(iv) Criteria/principles for selecting projects for budget funding are defined in the State Budget Law and PIL, but they are not very precise, and lack a strong policy orientation, as far as selecting new projects is concerned. Their application is also, in practice, not very transparent or consistent.

(v) Project management arrangements in Vietnam are generally sufficiently robust, but there is scope for further strengthening and for addressing weaknesses in application, particularly at the province level.

(vi) Further work needs to be done so that the MPI is in a position to be able to identify projects with a high risk of delivery failure and then to ensure that adequate steps are being taken to bring them back on track and, in the worst cases, close them down.

(vii) Shortfalls in funding for recurrent expenses are frequent, especially with respect to maintenance expenditures, which are consistently under-funded.

(viii) While Vietnam has now established a firm legal and regulatory basis for ex post evaluation, impact assessments are rarely performed, except for ODA projects, and lesson learning is not a strong feature of completion reports.

Reversing inefficiencies will require addressing the performance of public investment management at three levels:

- Improving allocative efficiency - meaning channeling of resources towards programs/projects with higher social returns and in line with government priorities;

- Enhancing productive efficiency - meaning efficient conversion of capital spending into infrastructure; and

- Using assets - new and existing - more efficiently.

The 8 must-have functions (and the specific institutional arrangements associated with them) identified in the WBG PIM diagnostic framework will, when performed effectively, deliver more efficient public investment at each of these levels.

In addition, transitional measures for dealing with the cumulative effects of inefficiencies will be required in order to create the right conditions for efficiency-enhancing improvements to succeed. 
Fiscally unsustainable programs of on-going projects, excessive arrears, non-performing projects and capacity constraints represent blockages to PIM reform that need to be addressed before good practice solutions can be fully implemented.

\section{Improving Allocative Efficiency}

Achieving improvements in the allocative efficiency of public investment will require:

- Developing affordable investment plans ('Affordability');

- Selecting technically, economically and socially feasible projects for funding in line with national and sectoral strategic priorities ('Selectivity'); and

- Ensuring predictable funding of selected projects ('Predictability').

These indicators of success in public investment management - which are captured under Strategic Guidance, Appraisal, Independent Review and Selection in the WBG assessment framework are not yet being adequately met in Vietnam. Strategic investment plans - which are often lists of unprioritized projects generated from bottom-up requests - lack fiscal realism and suffer from variable quality, particularly in relation to explanations of the underlying cause-effect relations linking outputs and desired outcomes. The introduction of medium-term investment plans (MTIPs) through the Public Investment Law (PIL) is a step in the right direction. However, the positive impact of this new instrument is frustrated by the absence of a cohesive fiscal framework for the plans, which would ensure their affordability, and by the large backlog of slow-moving on-going investment projects, which were developed pre-PIL. The introduction of on-lending and the debt ceiling further complicate the smooth implementation and functioning of the newly adopted changes. The ex-ante review of the repayment capacity of a province for each project proposal, in cases where borrowing is needed, can lead to unnecessary delay, in particular in the current context of asymmetric access to provincial debt data.

When it comes to the quality control of individual projects before they are selected for funding - the 'quality-at-entry' process, as it is sometimes known - weaknesses remain, even though the PIL has taken the country closer towards good practices. An early decision, the investment intention decision, on whether to proceed further with the assessment of a project and include it in the MTIP is a positive feature of the new process. Independent review of this decision by the MPI and MOF, at least for the largest projects, is also a positive step, but its potential impact is diminished by the narrow scope of the review, which is limited to affordability questions. The absence of an external perspective on the strategic relevance, rationale and social worth of a project is a weakness that potentially allows low priority or ill-conceived projects to proceed to the next stage of development. The rarity with which project alternatives are considered, the failure to identify risks and the limited attention to demand are also weaknesses in selectivity at this stage of PIM.

Selectivity is further impaired by the rigidity of the investment intentions decision, whereby project costs are 'locked in' at an early stage, often precluding updating on the basis of more in-depth design work. In addition, once a project features in the MTIP, on the basis of the investment intentions decision, it becomes difficult to dislodge from the investment program, even should contrary evidence concerning viability become available. This diminishes the importance of the investment decision at appraisal stage, when the feasibility study findings should come into play. This is an important shortfall compared to good international practice, where appraisal is a critical filter in the quality at 
entry process. Furthermore, the MPI has a restricted role in reviewing appraisal findings, thus forsaking an opportunity for objective assessment of any inherent optimism bias in the project proposal at this stage. Standardised methodologies for appraisal and systematic building of capacity in the application of such methodologies are also missing, further weakening quality at entry.

In good practice systems, project identification, design and appraisal are assisted by feedback on the outcomes of similar projects. Basic completion review, involving analysis of factors for success and failure, and expost impact evaluation examining broader developmental effects are not operational to any meaningful extent in Vietnam, although foreseen in the PIL. This further undermines the strength of selectivity.

The power to reject budget requests for projects that have not passed through the necessary assessment steps or been subject to the necessary decisions provides the discipline for an effective quality at entry process. At present, no central entity has a strong mandate to perform this important 'gate-keeping' function, which would usually be the responsibility of the organisation responsible for preparing the capital budget.

MTIPs have the potential for improving the predictability of financing for projects that are selected for funding, but there are drawbacks. MTIPs have been introduced in the context of an excessive portfolio of on-going projects, with no transition period and, until recently, no explicit design for linking them (and the annual investment plans deriving from them) to the medium-term and annual budgeting exercises. There is also an inherent difficulty in linking fixed-term investment plans with a rolling medium-term budgetary framework (as foreseen in the 2015 State Budget Law and currently being introduced), which is not easy to bridge. This makes the emergence of a gap between plans and budgets - with consequences for predictability - all but inevitable.

Predictability requires good knowledge of multi-year commitments in order to ensure financing of on-going projects, but at present, the central systems for collecting and analysing timely information on outstanding financial commitments are poorly developed. Even if such information were available, the budgetary process is not yet set up in such a way as to allocate funding in a systematic way to ongoing projects before consideration of funding requests for new projects.

Improving allocative efficiency will involve addressing affordability, selectivity and predictability dimensions of public investment management through implementation of the following overall recommendations, with linkages to the relevant functionalities in the WBG diagnostic framework in parentheses:

1. Improve long-term strategic planning of major infrastructure [Strategic Guidance].

2. Reinforce the role of the MPI and mainstream the review by the MOF in first-level screening of large-scale projects with significant fiscal impacts [Strategic Guidance].

3. Ensure PIM system design provides for the reversibility, at appraisal, of a positive investment intention decision [Appraisal].

4. Make the investment decision the critical decision of the 'quality-at-entry' processes [Appraisal].

5. Create the necessary regulatory, technical and capacity conditions for making social costbenefit analysis the default analytical tool for appraisal decisions [Appraisal].

6. Design and implement a strengthened independent review function, focusing on large-scale projects [Independent Review]. 
7. Situate project selection and budgeting in a realistic medium-term budgetary perspective [Selection].

8. Move towards a budgeting process that locks in funding for on-going projects before allocating to new projects [Selection].

In addition, the rationalisation of the existing portfolio of on-going projects, involving difficult decisions about dropping failing, irrelevant and/or poorly conceived projects, will be a pre-condition for these reform measures to succeed.

\section{Improving Productive Efficiency}

Achieving productive efficiency in public investment requires:

- Ensuring that projects approved for funding are ready for implementation ('Deliverability');

- Applying management systems and incentives to ensure that projects are constructed and operational on time, to budget and to specification ('Manageability'); and

- Identifying emerging problems early and implementing well informed and timely responses, including readiness to cancel projects ('Reactivity').

These indicators of productive efficiency are examined under Implementation, Adjustment and Operation in the WBG diagnostic framework. The deliverability of projects in Vietnam is highly variable. Criteria relating to implementation readiness are not defined or applied during budgeting, and projects with critical preparation gaps - inadequate attention to arrangements for land acquisition being a notable example - are often approved for funding. Too frequently, start-up of projects with approved funding can be delayed or implementation interrupted because of weaknesses in preparation, design and implementation planning. The absence of a structured learning process from the experience of implementing completed projects also means that lessons are not incorporated into implementation plans for new projects, leading to repetition of systemic problems.

The abrupt implementation of MTIPs, with limited time for attention to the deliverability of the plans and of their component projects, particularly in the face of absorptive capacity constraints and the backlog of on-going projects, is also causing transitional problems.

When it comes to the manageability of projects, organisational arrangements for managing and overseeing implementation are not systematised, and there are no formal guidelines on project management. For individual projects, there are also no requirements to establish formally the day-to-day operational procedures to be followed by the project team, in a project operational manual, for example.

The value for money advantages of competitive procurement are being dissipated because of the too frequent use of derogations from open, competitive methods, even though this is the default method. There is therefore scope for making further efficiency gains in this area.

Vietnam does not currently have a fully functioning PIM information system for centralised collection and management of information on implementation progress, as a basis for monitoring, although important efforts are being made to establish such a system. Responsive monitoring - whereby projects are followed closely, major problems detected early and corrective action taken and followed up - is taking place at a decentralised level, closer to project implementation, but is not yet well developed 
at the level of the MPI. The legal framework has improved through the PIL and the Construction Law, and the MPI is making efforts to improve the information base through the recently rolled-out PIM information system. There is, however, further work to be done before the MPI is in a position to be able to identify projects with a high risk of delivery failure and then to ensure that adequate steps are being taken to bring them back on track and, in the worst cases, close them down. Substantial gains from improving reactivity are therefore envisagable.

Improving productive efficiency will involve addressing deliverability, manageability and reactivity dimensions of public investment management through implementation of the following overall recommendations, with linkages to the relevant feature of the WBG diagnostic framework in parentheses:

9. Strengthen project management arrangements and lesson learning [Implementation].

10. Improve value for money in procurement by making greater use of competitive procurement methods [Implementation].

11. Strengthen centralized monitoring, focusing on major projects which are failing or at risk of failing [Adjustment].

12. Systematically re-appraise problem projects defined on the basis of quantitative triggers [Adjustment].

Similar to improvements in allocative efficiency, raising productive efficiency will also involve addressing transition issues related to the introduction of the MTIP and managing the backlog of prePIL on-going projects.

\section{Improving the Efficient Use of Assets}

Using capital assets more efficiently requires:

- Providing sufficient financial resources for operating and maintaining new and existing assets on a sustainable basis ('Sustainability').

- Registering assets systematically in databases and keeping records of values and condition up to date ('Custodianship').

In the WGB assessment framework, efficient use of assets is examined under the Operations function. Sustainability in Vietnam suffers from lack of synchronisation between the planning of new capital investment and the planning of operating and maintenance expenditures. The system of dual budgeting of capital and recurrent expenditures in Vietnam impedes integrated planning, and is further compounded by the absence of any requirement to demonstrate the recurrent expenditure implications of budget requests for capital projects. Overall maintenance allocations are significantly below what would be required to prevent premature deterioration, with negative consequences for service delivery and rehabilitation needs.

Registration of assets on centralised asset management systems and subsequent condition monitoring and revaluation remain poorly developed, with partial progress only in certain sectors, e.g., national roads.

Improving efficient use of assets will involve addressing sustainability and custodianship issues through implementation of the following overall recommendations, with linkages to the relevant feature of the WBG diagnostic framework in parentheses: 
13. Integrate more closely the budgeting of capital and recurrent expenditures [Selection/ Operation].

14. Improve the sustainability of investment projects by raising maintenance allocations closer to international standards [Operation].

15. Extend the use of modern asset management tools across government, beginning with the most important infrastructure categories [Operation].

\section{Cross-Cutting Issues}

Some of the recommendations of this report will mean a stronger role for the MPI, and in some instances for the MOF. In particular, the wider scope of scrutiny at first-level screening (investment intention decision), independent review of the basis for investment decisions and strengthened central monitoring will all require a deeper involvement from the MPI. In addition, a central body needs to coordinate and promote lesson learning from ex post review, once established. These are all critical functions, which the MPI is best placed to perform.

Reinforcing and broadening the role of the MPI must be carefully designed and sequenced so that new tasks can be performed efficiently and effectively, without hindrance to the smooth running of the PIM system as a whole. This will mean designing in detail, and then piloting, improved processes before their full enactment, rather than regulating for change before the necessary procedures and tools are in place and tested. It will also require developing the necessary capacities before full implementation, so that administrative bottlenecks are not created, putting the reforms into disrepute.

The envisaged role for the MPI is deliberately contained to large-scale projects, since central agencies cannot realistically be directly involved in all projects because of capacity and time limitations. For lower value projects, the MPI's role should be one of developing systems and methods and overseeing and supporting their application. 


\section{Introduction: Context, Rationale and Methods}

\section{Context}

1. Vietnam's on-going reform program places an emphasis on improved efficiency of public investment in order to boost the country's economic performance, which remains highly dependent on factor accumulation. Investment by the state sector has been the most important source of capital accumulation in Vietnam despite rapid growth of the domestic private sector and FDI since the start of economic reform in the late 1980s. Investment from the state budget is the dominant form of state investment and accounts for more than one quarter of total investment.

2. The urgency of reform is a result of growing concern about the rapidly declining efficiency of public investment. The allocation of capital investment lacks strategic priorities and is spread too thinly in many areas. Official Government reports note that there are about 40,000 on-going projects with the majority being managed in provinces. A recent MPI portfolio review of 2013-15 shows that resources allocated from the state budget could only accommodate less than one half of total investment needs from both central and local governments for already approved projects. Further evidence of inefficiency is the level of capital expenditure arrears, which remains high and relatively unchanged even during the recent period of investment consolidation.

3. The recent consensus on reform of public investment appears to have benefited from the global economic crisis in 2008-09 and the Government's policy reaction, involving a significant stimulus package-boosting public investment even more. Both have contributed to exposing a number of deficiencies in Vietnam's public investment management (PIM) system, ranging from the gap between the legal and regulatory framework and practice on the ground; to misallocation of resources resulting from loopholes in the appraisal, budgeting, implementation, and evaluation of public investments. The two most recent five-year socioeconomic development plans (SEDP 2011-15 and SEDP 2016-20) acknowledge the critical inefficiency of public investment, especially significant overinvestment and fragmentation, and envisage a reform agenda to consolidate public investment and raise the quality of the PIM system with a target of reaching the top four of ASEAN by 2020.

4. Strengthening the legal framework is a cornerstone of the reform program for restructuring the management of public investment. Vietnam has to date made commendable progresses in establishing a sound public financial management system, equitizing state-owned enterprises (SOEs), and enhancing the legal framework for public investment management. The recent enactment and amendment of a number of laws including the Public Investment Law (PIL), Procurement Law, Construction Law (CL), State Budget Law, and the Law on State Capital Invested in Enterprises are critical achievements in moving towards international good practices. The PIL and $\mathrm{CL}$ introduced several regulatory improvements for the entire project cycle. Notable changes include the introduction of medium-term investment planning, strengthening the appraisal of investment intentions, professionalization of PMUs, and enhancing the role of MOF and MPI in project selection. 


\section{Rationale for the PIM Assessment}

5. Despite the progress made by the Government in recent years including the issuance of the Public Investment Law, progress in restructuring of public investment has been slow and mostly focuses on cleaning up the existing fragmented portfolio. Many measures are short-term in nature and aim to deal quickly with consequences rather than tackle the root causes of the problems. There is also no evidence-based systemic and comprehensive blueprint for improving the efficiency of public investment over the long term.

6. Vietnam continues to face challenges in each of the three dimensions that contribute to the overall efficiency of public investment, namely, allocative efficiency, productive efficiency, and efficient use of assets. Allocative efficiency requires that projects are prioritized and selected according to their social worth and in alignment with government's strategic priorities, consistent with available fiscal resources over the medium term. Achieving productive efficiency in investment demands that, once the decision is taken to go ahead with a project, inputs are procured economically and the construction process is managed optimally, so that the infrastructure assets are completed on budget, on time and to specification. Efficient use of assets is achieved when adequate financing is provided to ensure infrastructure services are delivered as intended and to maintain the assets for their planned lives and service quality.

\section{Vietnam faces a number of efficiency challenges, which can be summarized as follows:}

- Inadequate safeguards to prevent financial resources allocated to weak, low priority and/or unaffordable projects;

- Suboptimal use of resources for investment once allocated, leading to cost overruns, delays and departures from specification;

- Under-provision of complementary resources required to achieve service delivery targets for the assets created; and

- The cumulative effects of earlier inefficiencies leading to blockages, such as over-extended investment programs and arrears, which undermine well-intentioned reforms, even if these make sense in isolation.

8. A successful PIM reform program will need to address these challenges by delivering on each of the three dimensions of efficiency. The main indicators of success for such a program would be:

- Allocative Efficiency

- Affordable investment plans - affordability

- Feasible projects selected for funding in line with national and sectoral strategic priorities - selectivity

- Predictable financing of prioritized projects - predictability

- Productive Efficiency

- Approved projects ready for implementation - deliverability

- Construction on time, to budget and to specification - manageability

- Timely and informed responses to implementation problems - reactivity 
- Efficient Use of Assets

- Adequate resources for operations and maintenance - sustainability

- Systematic registration and management of assets - custodianship

9. A major factor behind the slow progress in addressing inefficiencies and restructuring public investment is the lack of a well-designed reform program with clearly defined objectives and priorities. While the decision to overhaul the PIM system had already been made six years ago, no specific reform program has yet been developed. Effective implementation of the newly promulgated legal documents also requires considerable work to guide their application, especially in highly decentralized settings. In spite of recent improvements, public investment continues to be governed by a complex and fragmented regulatory framework, which includes a large number of laws.

10. It is against this background that the Ministry of Public Investment (MPI) requested the World Bank to undertake a diagnostic assessment of Vietnam's public investment management (PIM) system. The aims of the assessment are to identify weaknesses compared with good practice models, identify priorities for reform and make specific recommendations on actions over the short, medium and long term.

\section{Approach to the Diagnostic Assessment}

11. The PIM assessment has been prepared following the World Bank Group's PIM Diagnostic Framework as formalized in the 2014 publication, 'The Power of Public Investment Management' ${ }^{\prime}$. This framework analyzes the presence and quality of institutional arrangements required to support performance of eight "must-have functions" across the project and capital budgeting cycles $^{2}$, thus enabling a robust assessment against good international practice. The framework yields a qualitative assessment of a country's PIM system, rather than an indicator-based assessment (similar to PEFA). Box 1 below summarizes each of the "must-have functions".

12. Achieving efficiency in public investment depends on the existence of satisfactory institutional arrangements - legal/regulatory framework, organizational structures, capacities and incentives - to perform the functions described in Box 1 in an effective way. The 8 musthave functions (and the specific institutional arrangements associated with them) identified in the WBG PIM diagnostic framework will, when performed effectively, deliver more efficient public investment, working through improved allocative and productive efficiency and more efficient use of assets. Strategic guidance, appraisal, independent review and selection relate to allocative efficiency. Implementation and adjustment relate to productive efficiency. Operation relates to efficient use of assets. Evaluation contributes to improving efficiency across three dimensions.

1 'The Power of Public Investment: Transforming Resources into Assets for Growth', Anand Rajaram, Tuan Minh Le, Kai Kaiser, JayHyung Kim, and Jonas Frank, Editors, World Bank, 2014

2 The eight must have functions are: investment guidance, formal appraisal, independent review, project selection and budgeting, project implementation, project adjustment, facility operation, and completion review and evaluation 


\section{Box 1: Eight Must-Have Features of a Good PIM System}

The World Bank PIM diagnostic identifies eight functional stages of the PIM cycle and, based on country experience, and suggests desirable features for each stage in order to achieve PIM efficiency. Each of these features is described as follows:

Stage 1: Strategic Guidance and First Level Screening: National and/or sector strategy documents are specific enough, and have sufficient coherence and authority to actually guide public investment, and are used systematically to screen new projects (with at least some projects dropped at the preliminary screening stage).

Stage 2: Project Appraisal: Project development follows a standardized and well-defined set of procedures, and projects are appraised using the full range of techniques as appropriate. There are comprehensive central guidelines on project appraisal, including specific detailed guidance on the appraisal of PPPs.

Stage 3: Independent Appraisal Review: The risk of line ministries "cooking the numbers" to ensure that a project passes appraisal is limited by an independent review of the project. This is a key feature which can be implemented in various ways and with various degrees of "independence" of the entity in charge of the reviews.

Stage 4: Project Selection and Budgeting: In general, only projects that have been subject to thorough appraisal, and have been independently reviewed, are selected for funding in the budget. Multi-year budget authority with adequate financing and funding predictability supports effective project implementation.

Stage 5: Project Implementation: There is a strong focus on managing the total project costs over the lifetime of each project. Clear roles and responsibilities are in place for project implementation, with regular reporting on financial and non-financial progress and close monitoring by the relevant Central Agencies. Sound procurement systems are in place and are consistently implemented, with advanced techniques for allocating risks between government and contractor.

Stage 6: Project monitoring and adjustment: Specific mechanisms are in place to trigger a review of a project's continued justification if there are material changes to project costs, schedule, or expected benefits.

Stage 7: Facility Operation and Maintenance: Financing and other resources for operation of the facility is planned for and made available by the relevant authority. Comprehensive and reliable asset registers are maintained and are subject to external audit.

Stage 8: Basic Completion Review and Evaluation: All advanced countries put significant effort into ex post review. Investment projects are subject to audit by the supreme audit institution, including value-formoney audits.

Source: Based on The Power of Public Investment Management, World Bank, 2014, with adaptations by the authors.

\section{The PIM assessment is based on a triangulation of findings based on the following main data sources:}

- Desk reviews of relevant laws, regulations and procedures, as well as previous diagnostic reports on Vietnam and international experiences;

- Interviews with key stakeholders across government; and

- Evidence on procedures used in a sample of public investment projects from the transport and education sectors. 
14. A desk review of previous diagnostic studies and of legal and regulatory documentation was carried out. The following documents were reviewed:

- State Budget Law 2015;

- Public Investment Law 2014 and related secondary legislation;

- Construction Law 2014 and related secondary legislation;

- Procurement Law 2013 and related secondary legislation; and

- Decree 15/2015 (on PPP).

15. Key stakeholders across government were interviewed to validate findings from desk reviews. Within the Ministry of Planning and Investment, discussions were held with the Departments of:

- National Economic Issues;

- Appraisal and Monitoring;

- Local Economy;

- Science, Natural Resources and Environment; and

- Infrastructure and Urban Development.

In addition to the relevant internal departments of the Ministry of Planning and Investment, interviews were also held with the Ministry of Finance (MOF - Investment Department), Ministry of Transport (MOT), the Ministry of Education and Training (MOET) and the Ministry of Construction (MOC). A visit to a representative province, Bac Giang, was also undertaken, where meetings were held with the Departments of Finance, Planning, and Transport. The Bac Giang project management unit (PMU) for transport projects also joined the meeting with the Department of Transport.

16. With the agreement of the MPI, the Transport and Education sectors were selected for the case studies, because of their contrasting in service delivery and hence investment management. To complement the interviews and written documentation on regulations and procedures, a project-level evidence base was assembled, based on a sample of 17 on-going or completed public investment projects as case studies. Despite a relatively small sample size, and therefore the reduced statistical significance of findings, project-level information has been triangulated against the other data sources, to either confirm findings or indicate areas for further investigation or clarification.

17. Criteria for selection of the project sample were established in collaboration with the Ministries of Planning, Transport, and Education and Training to reflect mainly national procedures and processes. The sample includes a range of on-going or recently completed projects funded from a range of funding sources - ODA, bonds and state budget. The sample frame of projects were provided by the MPI based on the portfolio of on-going or recently-completed projects for Transport and Education (10 and 7 projects, respectively). A profile of the project sample is given in Tables 1 and 2 below.

18. In addition to the interviews of PMUs of the selected projects, interviews were also held with representatives of MOT and MOET to get their responses about a hypothetical "representative" project under the ministry's management. Responses for these "representative" projects were taken into account in the report's analysis together with PMUs' responses to overcome the weaknesses of small samples as indicated above. 
Table 1: Sample of Projects from the Transport Sector

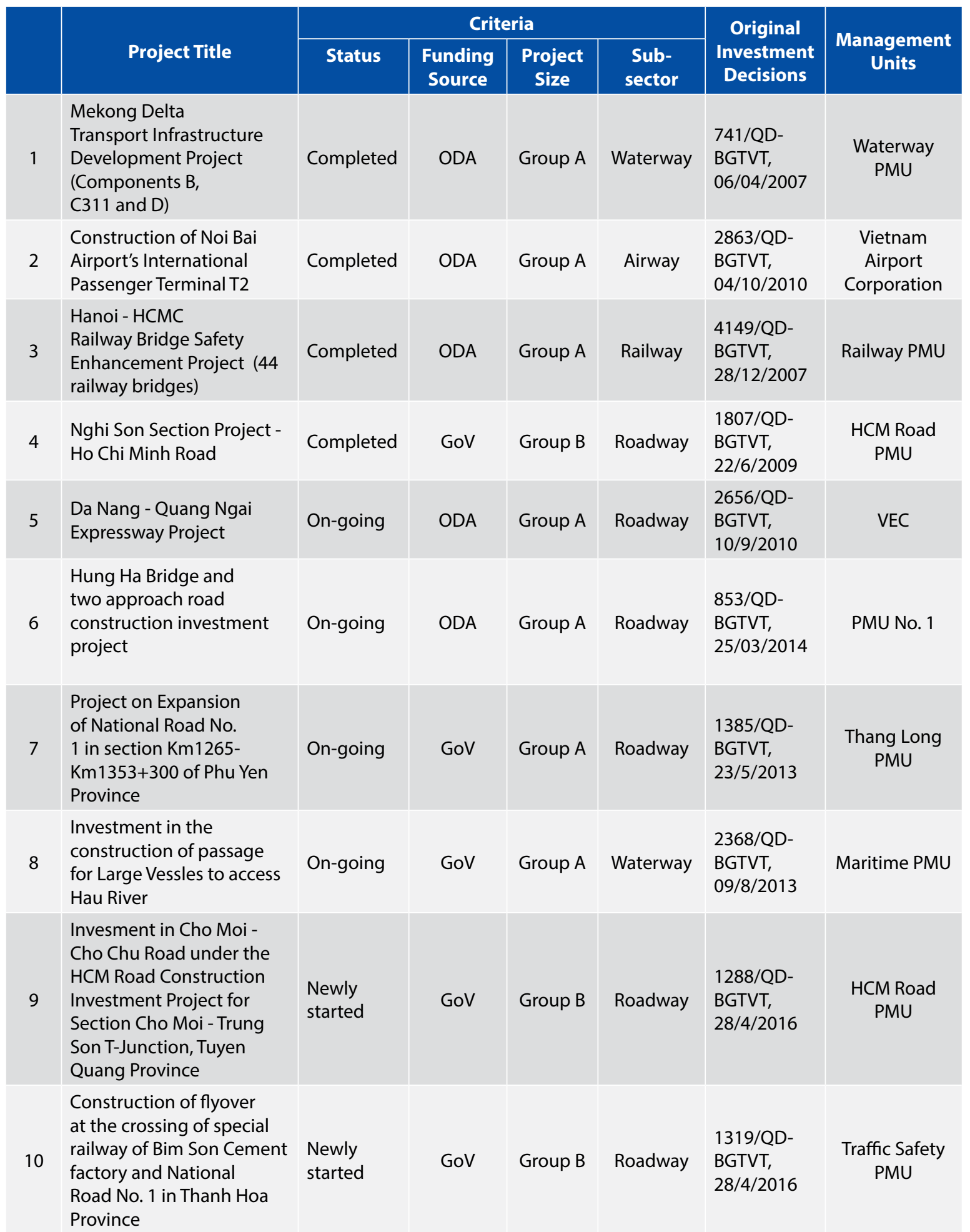


Table 2: Sample of Projects from the Education Sector

\begin{tabular}{|c|c|c|c|c|c|c|c|}
\hline & \multirow[b]{2}{*}{ Project Title } & \multicolumn{4}{|c|}{ Criteria } & \multirow{2}{*}{$\begin{array}{l}\text { Original } \\
\text { Investment } \\
\text { Decisions }\end{array}$} & \multirow{2}{*}{$\begin{array}{c}\text { Management } \\
\text { Units }\end{array}$} \\
\hline & & Status & $\begin{array}{l}\text { Funding } \\
\text { Source }\end{array}$ & $\begin{array}{l}\text { Project } \\
\text { Size }\end{array}$ & $\begin{array}{l}\text { Sub- } \\
\text { sector }\end{array}$ & & \\
\hline 1 & $\begin{array}{l}\text { Training Center Building } \\
\text { Project - National } \\
\text { Economics University }\end{array}$ & On-going & $\begin{array}{l}\text { GoV and } \\
\text { others }\end{array}$ & Group A & $\begin{array}{l}\text { Training } \\
\text { center } \\
\text { buiding }\end{array}$ & - & $\begin{array}{l}\text { Joint Stock } \\
\text { Company of } \\
\text { Construction } \\
\text { Technology } \\
\text { and Equipment } \\
\text { CONINCO }\end{array}$ \\
\hline 2 & $\begin{array}{l}\text { Lecture Hall - National } \\
\text { Institute of Education } \\
\text { Management }\end{array}$ & On-going & $\begin{array}{l}\text { GoV and } \\
\text { others }\end{array}$ & Group B & $\begin{array}{l}\text { Lecture } \\
\text { Hall }\end{array}$ & $\begin{array}{l}\text { 4957/QD- } \\
\text { BGDDT, } \\
30 / 10 / 2015\end{array}$ & $\begin{array}{l}\text { Project } \\
\text { Management } \\
\text { Board of MOET }\end{array}$ \\
\hline 3 & $\begin{array}{l}\text { Viet Duc University } \\
\text { Project (New Model } \\
\text { University Project - } \\
\text { NMUP }\end{array}$ & On-going & $\begin{array}{c}\text { ODA and } \\
\text { GoV }\end{array}$ & Group A & $\begin{array}{l}\text { Training } \\
\text { center } \\
\text { buiding }\end{array}$ & - & $\begin{array}{l}\text { - Viet Duc } \\
\text { University } \\
\text { PMU under } \\
\text { the Ministry of } \\
\text { Education and } \\
\text { Training } \\
\text { - Viet Duc } \\
\text { University PMU } \\
\text { under VMU }\end{array}$ \\
\hline 4 & $\begin{array}{l}\text { Project to build lecture } \\
\text { halls and practice } \\
\text { workshops in F1 Zone } \\
\text { of the Ho Chi Minh City } \\
\text { University of Technology } \\
\text { and Education. }\end{array}$ & On-going & $\begin{array}{l}\text { GoV and } \\
\text { others }\end{array}$ & Group B & $\begin{array}{l}\text { Lecture } \\
\text { Halls }\end{array}$ & - & $\begin{array}{l}\text { Project } \\
\text { Management } \\
\text { Board of MOET }\end{array}$ \\
\hline 5 & $\begin{array}{l}\text { Investment in lecture } \\
\text { halls and a multipurpose } \\
\text { hall at the campus } 2 \text { - Ho } \\
\text { Chi Minh City University } \\
\text { of Law }\end{array}$ & Completed & $\begin{array}{l}\text { GoV and } \\
\text { others }\end{array}$ & Group B & $\begin{array}{l}\text { Lecture } \\
\text { halls }\end{array}$ & - & $\begin{array}{l}\text { Ho Chi Minh } \\
\text { City University } \\
\text { of Law }\end{array}$ \\
\hline 6 & $\begin{array}{l}\text { Constructing the Kien } \\
\text { Giang campus of Nha } \\
\text { Trang University }\end{array}$ & On-going & $\begin{array}{l}\text { GoV and } \\
\text { others }\end{array}$ & Group A & $\begin{array}{l}\text { Training } \\
\text { center } \\
\text { buiding }\end{array}$ & - & $\begin{array}{l}\text { Department for } \\
\text { Infrastructure } \\
\text { and Teaching } \\
\text { Equipment } \\
\text { (MOET) }\end{array}$ \\
\hline 7 & $\begin{array}{l}\text { Investment in technical } \\
\text { infrastructure in Long } \\
\text { Binh Campus - Open } \\
\text { University Ho Chi Minh } \\
\text { City (The first period) }\end{array}$ & Completed & $\begin{array}{l}\text { GoV and } \\
\text { others }\end{array}$ & Group B & $\begin{array}{l}\text { Training } \\
\text { center } \\
\text { buiding }\end{array}$ & $\begin{array}{l}\text { 8296/QĐ- } \\
\text { BGDĐT, } \\
\text { 18/11/2009 }\end{array}$ & $\begin{array}{l}\text { Department for } \\
\text { Infrastructure } \\
\text { and Teaching } \\
\text { Equipment } \\
\text { (MOET) }\end{array}$ \\
\hline
\end{tabular}

19. To assist in analyzing the selected projects in a consistent way, a detailed questionnaire was developed. Aside from background information on the project value, scope, duration, cost and financing of the projects, the questionnaire includes a set of questions structured around the World Bank diagnostic approach to the procedures used throughout the main stages of the PIM cycle. The questionnaire is presented as Annex 3 to this report. The responses to the questionnaires were validated by interviews with representatives of the sampled projects. 


\section{Structure of the Report}

20. In Section 3, the report briefly reviews the capital expenditure trends and patterns in the last one and a half decades. To provide an overview of Vietnam's public investment and PIM, the section presents some snap shots of capital investment funding allocations and sector differences. Capital spending trends and patterns funded by the state budget, a comparison between the central and provincial levels, and government bond capital, are analyzed. Section 4 presents an institutional mapping of PIM in Vietnam.

21. The findings of the case studies are brought into the analysis of the current PIM system as set out in Sections 5-12 of this report. In these eight sections, each is composed of three parts: (i) principles and lessons from good practices; (ii) the assessment and diagnostic; and (iii) the recommendations. Concluding remarks with a summary table of recommendations are included in the final section. 


\section{Capital Spending Trends and Patterns ${ }^{3}$}

22. To provide an overview of Vietnam's present situation, this section describes the overall trends observed in capital spending and the links to development priorities, the sectoral breakdown of investment allocations, the distribution of resources across levels of government, and how resources are mobilized through the issuance of government bonds, the proceeds of which are then distributed across sectors and tiers of government.

\section{Capital Spending Financed by the State Budget}

23. State capital spending has decreased in recent years but still remains moderately higher than other countries in the region. During 2011-2015, the share of public capital spending accounted for around one third of total investment in Vietnam. Capital spending (including Government bonds) financed by the state budget represented 13.1 percent of GDP in 2009 (Figure 1). However, by 2012, the budget investment/GDP ratio had regressed to its 2007-08 level and dropped further to $7.8 \%$ in 2014. The trajectory of capital spending coincides with the level observed across the East Asia region (Figure 2). However, the declining trend reflects the decrease of overall investment, and the share of state investment in total investment has remained relatively constant in the last 10 years.

Figure 1: State budget as share of total investment and GDP, 2006-15

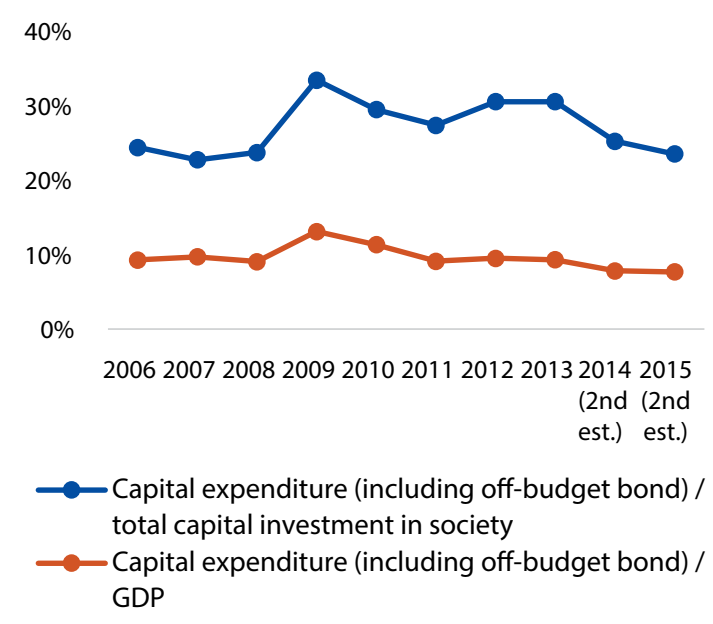

Source: MOF.

\section{Figure 2: Public investment in East Asia Pacific and OECD countries (percent of GDP), 2004-2011}

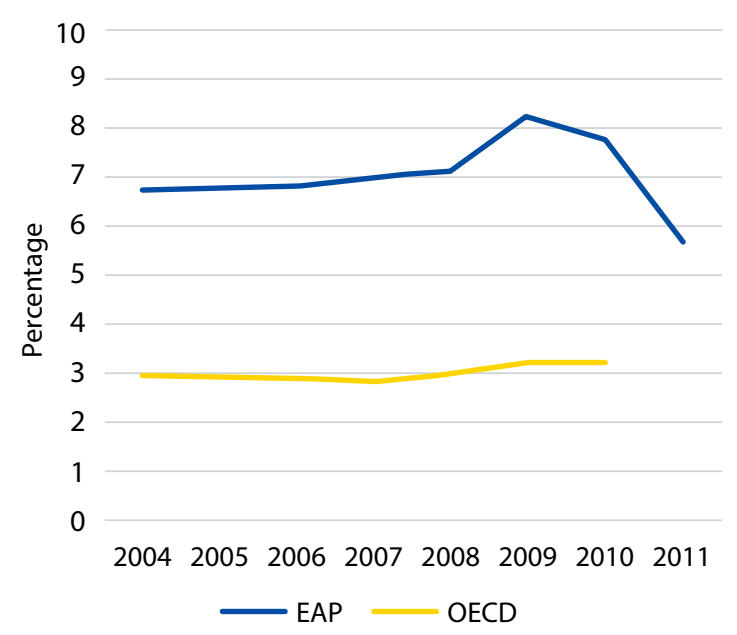

Source: IMF WEO Database 2014

24. The share of budget expenditure in state investment, however, exhibits an increasing trend. State investment, including the state budget, investment from SOEs, state credit, and government bonds, accounts for about $41 \%$ total investment. The state budget remains the largest source of financing for state investment and its contribution has increased since 2001 (Table 3). In 2011-15, the state budget provided 52 percent of the resources for public investment. This share was higher than that in the period 2006-10 and nine percentage points higher than 2001-05. SOEs represented

3 This section is taken from the Public Expenditure Review (PER) by the World Bank. 
the second most important source of financing for state investment, followed by state credit and government bonds. Significant increases in investment from the state budget and government bonds (about 10 percentage point each) were compensated by decreases in investment from SOEs and state credits. The increase in budgetary investment, however, was mainly observed in the first three years of the 2011 - 2015 plan period. The period 2014 - 2015 witnessed a substantial decline of the share of budget investment while SOE and bond financed investments picked up.

Table 3: Funding sources of public investment (percent), 2001-15

\begin{tabular}{|l|c|c|c|}
\hline & $\mathbf{2 0 0 1 - 0 5}$ & $\mathbf{2 0 0 6 - 1 0}$ & $\mathbf{2 0 1 1 - 1 5}$ \\
\hline State budget & 42.9 & 49.4 & 52.0 \\
\hline Investments from SOEs & 27.9 & 23.2 & 21.7 \\
\hline State credits & 26.2 & 14.7 & 12.2 \\
\hline Government bonds & 3.0 & 12.6 & 14.1 \\
\hline
\end{tabular}

Source: Five-year Development Plans for 2006-10, 2011-15.

25. The persistently large difference between budgeted and actual capital spending indicates the low reliability of the capital budget and at the same time, a lack of budget discipline in implementation. The gapjumped significantly in 2009 due to the fiscal stimulus and has stayed similarly large after the post-crisis decline in 2011. It is often expected that during a fiscal consolidation, public investment is more sensitive to cuts than recurrent expenditure, such as wages and social services. In Vietnam the contrary is the case: a combination of inflation, the increased cost of land acquisition and the depreciation of the exchange rate has led to a notable increase in the total investment costs of projects compared to the budget plan. In addition, the central and sub-national governments also made use of over-realized revenues and budget contingency headings for capital expenditure. As a result, the actual outturns were higher still than the originally planned capital expenditure (Figure 3 ). ${ }^{4}$

Figure 3: Difference between budgeted and actual capital spending (percent), 2006-15

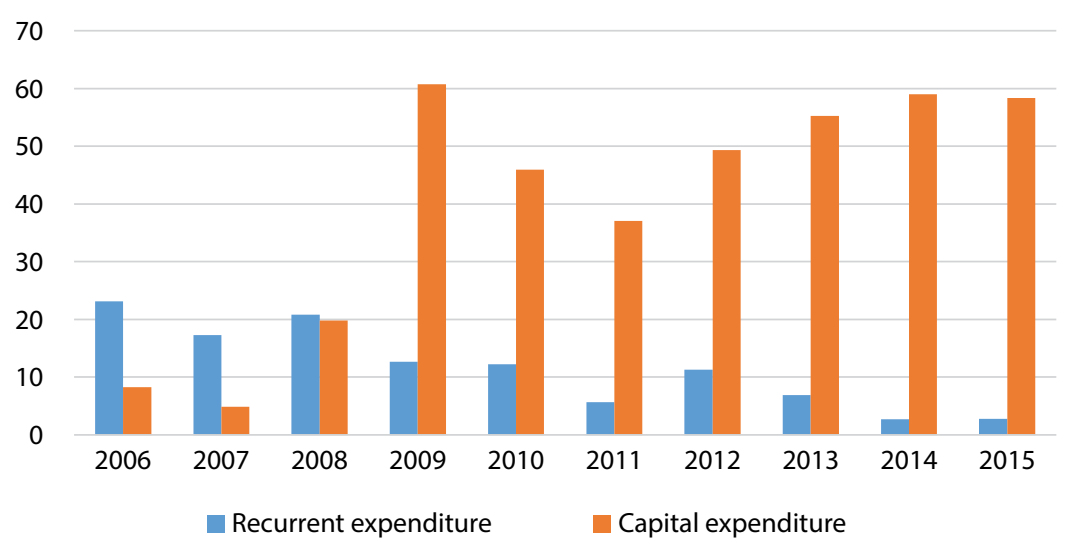

Source: Ministry of Finance.

4 The PIL aims to address this issue by reserving a contingency fund of $10 \%$ of the capital budget each year for emergency issues. 
26. The allocation of budget capital expenditure is significantly in favour of transport and agriculture sector. Between 2006 and 2014, allocations to transport and agriculture represented about 23 and 21 percent of total budget investment, respectively (Table 4). The share of the transport sector was increasingly topped up by FDI and domestic private investments, resulting impressive development in the sector recently.

Table 4: Budget capital spending by sector (VND billion), 2000-14

\begin{tabular}{|l|r|r|r|}
\hline \multicolumn{1}{|c|}{ Sector } & $\mathbf{2 0 0 0 - 0 5}$ & $\mathbf{2 0 0 6 - 1 0}$ & $\mathbf{2 0 1 1 - 1 4}$ \\
\hline Total & $\mathbf{2 2 1 . 2 1}$ & $\mathbf{4 6 0 . 7 2}$ & $\mathbf{6 2 9 . 4 8}$ \\
\hline Transport & 69.94 & 107.64 & 144.32 \\
\hline Agriculture, Forestry, Fishery and Irrigation & $(31.6 \%)$ & $(23.33 \%)$ & $(22.9 \%)$ \\
\hline Science and Technology, Education and Training, Health, & 47.50 & & 134.61 \\
\hline Culture, Sport & $(21.5 \%)$ & $(20.55 \%)$ & $(21.4 \%)$ \\
\hline National Defence, Public Security & $(29.8 \%)$ & $(37.5 \%)$ & 222.12 \\
\hline Other sectors & 7.93 & 14.14 & $25.3 \%)$ \\
\hline
\end{tabular}

Source: Ministry of Finance.

27. Investment priority has been given to the road and irrigation subsectors within transport and agriculture. Between 2009 and 2012, the share of roads in the transport sector averaged 91 percent of total investment (Figure 4). Similarly, the share of irrigation in the agriculture sector averaged 79 percent during the same period. This trend is observed at both national and subnational levels.

Figure 4: Sub-sectoral breakdown of capital spending in transport and agriculture sectors, 2009-2012
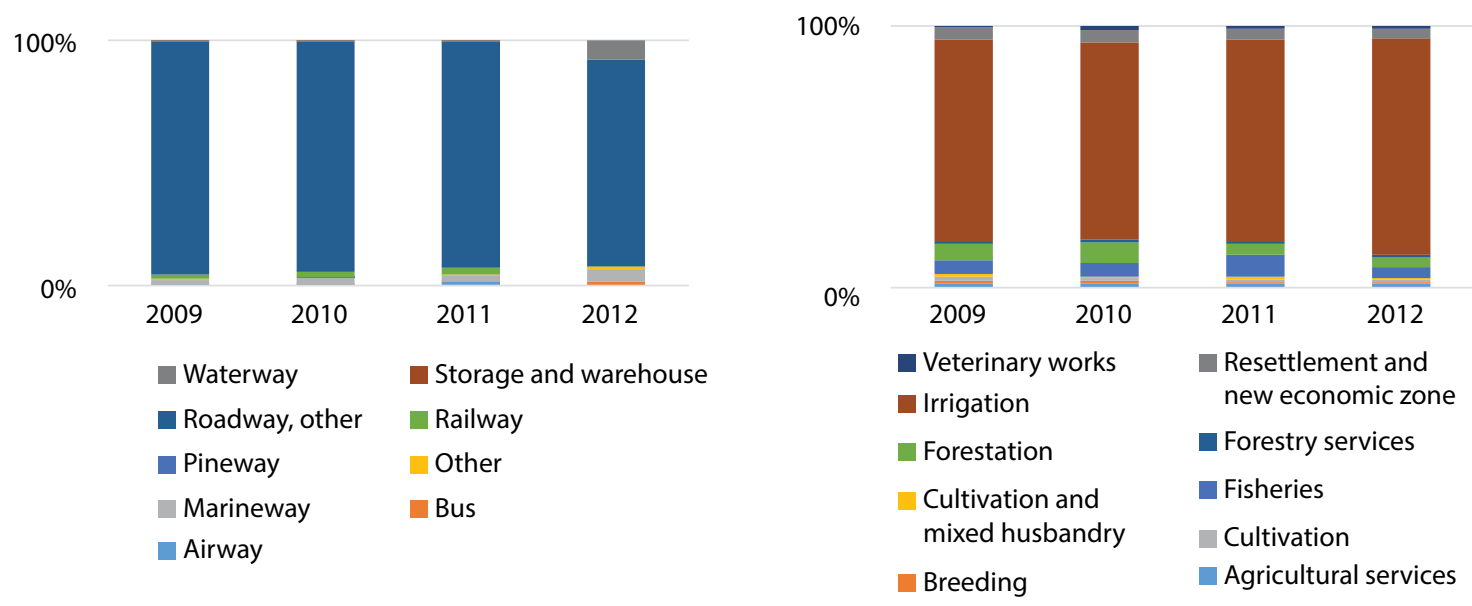


\section{Decentralization of Capital Spending Mandates and Authorities}

28. While spending has increased at both central and provincial levels, provinces now account for a greater share of the state capital budget. Decentralization of capital spending has steadily increased over the past decade (See Table 5). Public investment responsibilities were transferred to provinces as part of a broader decentralization policy initiated with Resolution 8/2004. As a result of this decision, responsibilities relating to socio-economic planning, management of natural resources, budgeting, SOE management, public service delivery, and capital spending were devolved to the provinces. Provinces were also granted decision-making authority over capital projects that were funded solely with provincial resources.

Table 5: Capital spending by tier of government and sector (VND billion), 2000-14

\begin{tabular}{|l|c|c|c|}
\hline \multicolumn{1}{|c|}{ Tier of Government } & $\mathbf{2 0 0 0 - 0 5}$ & $\mathbf{2 0 0 6 - 1 0}$ & $\mathbf{2 0 1 1 - 1 4}$ \\
\hline Total & $\mathbf{2 2 1 . 2 1}$ & $\mathbf{4 6 0 . 7 2}$ & $\mathbf{6 2 9 . 4 8}$ \\
\hline Central government & 86.14 & 115.05 & 137.02 \\
\hline Provinces & $(38.9 \%)$ & $(25 \%)$ & $(22 \%)$ \\
\hline
\end{tabular}

Source: Ministry of Finance.

29. Subnational public investment, represented approximately 78 percent of capital spending in the general budget for 2011-14 - one of the highest shares in the world - and made up 33 percent of provincial budgets. Public investment executed by central government has declined both as a share of total investment allocations and as a percentage of the national budget. Provincial authorities are responsible for identifying, appraising and approving investment funded through their budgets. Central government approves provincial investment plans and monitors them. The level of decentralization of capital spending is among the highest in the developing world. The average figure for EAP countries in 2010 was 40 percent, compared to $75 \%$ in Vietnam in 2006-10. This places Vietnam in the spectrum of federal and highly decentralized countries.

30. As a result of decentralization, provinces have overwhelming authority over all investment projects, except for nationally important projects. Central government has limited tools to influence allocations or monitor results of local investments beyond the approval of plans, master plans or appraisal of financial availability. Poor selection, in combination with soft budget constraints (for example, carry-over practices), has been one of the drivers of the multiplication of projects and the demand for resources, which are often beyond the capacity of the state budget to fund. 


\section{Government Bond Financing}

31. The impact of government bond capital, an important additional resource for infrastructure and social protection, has been diminished in recent years because the resources mobilized were spread too thinly across a large number of capital projects. Between 2003 and 2007, 614 transport and irrigation projects were financed, and 621 district and inter-district general hospitals were completed. By 2008-09, the number of projects significantly increased, spreading the funding more thinly over thousands of projects. As a result, many projects had to be cut, downsized, or delayed, resulting in waste of resources.

32. The absence of specific guidance for prioritizing allocations each year reduces the impact of the capital mobilized through government bonds. The intended goal is to prioritize investment for the transport and irrigation sectors of mountainous areas. However in practice, the government bond budget allocated for disadvantaged mountainous areas accounted for only a small share, lower than that of the lowland provinces. Specifically, the average amount allocated in the period 2012-15 for a province in the northern mountainous area was VND 1,779 billion, and that for a province in the Red River Delta was VND 2,329 billion. 



\section{An Institutional Mapping of PIM in Vietnam}

\section{Institutional Arrangements and Coordination in PIM}

33. The emphasis of the WBG PIM assessment framework is on functionality and not form. It is not therefore prescriptive when it comes to how an administration should organize itself to perform the 8 'must-have' functions, and indeed good practice countries organize themselves in different ways. The current section looks at how Vietnam regulates and is organized to carry out PIM-related functions. The purpose of the diagnostic part of the report is to explore whether and how effectively the required functions are being performed, in whatever way these are organized or classified in Vietnam.

34. The overall PIM system in Vietnam covers three core functions: investment planning, budgeting, and project management, which are inter-related and encompass elements of the 8 "must-have" functions of the WBG's PIM framework. These core functions target different aspects of PIM and are consequently led by different agencies. The cross-cutting nature of these core PIM functions requires the participation of various line ministries and provinces. Figure 5 depicts the main steps of each function, and the associated lead agencies. It also shows that all the core PIM functions must serve the development objectives and strategic priorities as set out by various approved strategy papers, such as the SEDS, Master Plans and SEDPs.

35. Vietnam has a dual budgeting practice in which capital budgeting is led by the planning sector whereas the medium-term and annual budget plans are led by the finance sector. The medium-term capital budget determines the overall level of capital expenditure and its allocation among sectors and provinces compatible with these targets. Medium-term budget estimates are derived based on the considerations of a number of factors including the medium-term development targets, resource mobilization capability, allocation norms ${ }^{5}$, as well as the medium-term debt management strategy. The annual budget elaborates the implementation of the medium-term financing plan and includes the allocation or disbursements for the preparation or implementation of specific investment projects or operating and maintenance (O\&M) costs of completed projects.

36. Investment planning has become a highly decentralized process with limited linkage to the budgeting process. The investment planning begins with a bottom-up process, in which the draft plan of each spending agency is a consolidated plan of inputs from the subordinated agencies. The MPI then consolidates draft plans of all line ministries, agencies, and provinces to produce a draft national plan. In the planning process, the prioritization and selection of projects between and within sectors/sub-sectors are undertaken by the agency, who "owns" the project. The budget aspect of investment planning comes late in the process when the MPI and the MOF produce the budget framework and review the draft investment plans of spending agencies to make sure that demand for funding from the central government is compatible with the national capital budget. The planning sector also assumes the leading role in the monitoring and evaluation (M\&E) of public investment projects.

5 Vietnam has introduced allocation norms for a formula-based capital balancing transfers from 2006. The norms are formulated every five years, and used for the calculation of capital transfers from central to provincial governments for the corresponding SEDP. The allocation norms include indicators from various categories such as population, level of provincial development, and provincial domestic revenue. Provinces have also formulated their own norms for capital transfers to lower levels. 
37. The third core function, project cycle management, belongs primarily under the responsibilities of the spending agencies. Vietnam has a decentralized system for project management in which the institutional arrangements for decision-making depend on the category of projects. The appraisal and approval of nationally important projects involve the Prime Minister and the National Assembly, respectively. However, the vast majority of projects belongs to categories $A$, $B$, and $C$ and, until recently, the central ministries (MPI and MOF) only had limited involvement in the upstream part of these projects' cycle. From the implementation stage, MPI and MOF only play a minor role, apart from the allocation of annual budget. Critical changes in the project preparation process for projects in category A, which were recently introduced through the 2014 Public Investment Law (PIL) are depicted in Figure 6. Similar procedures are adopted for projects Category B, which, however, do not require a prefeasibility study (PFS) and only need approvals from line ministries or provincial PPCs. Projects $C$ are much less affected by these changes.

Figure 5: Core Functions of Public Investment Management in Vietnam

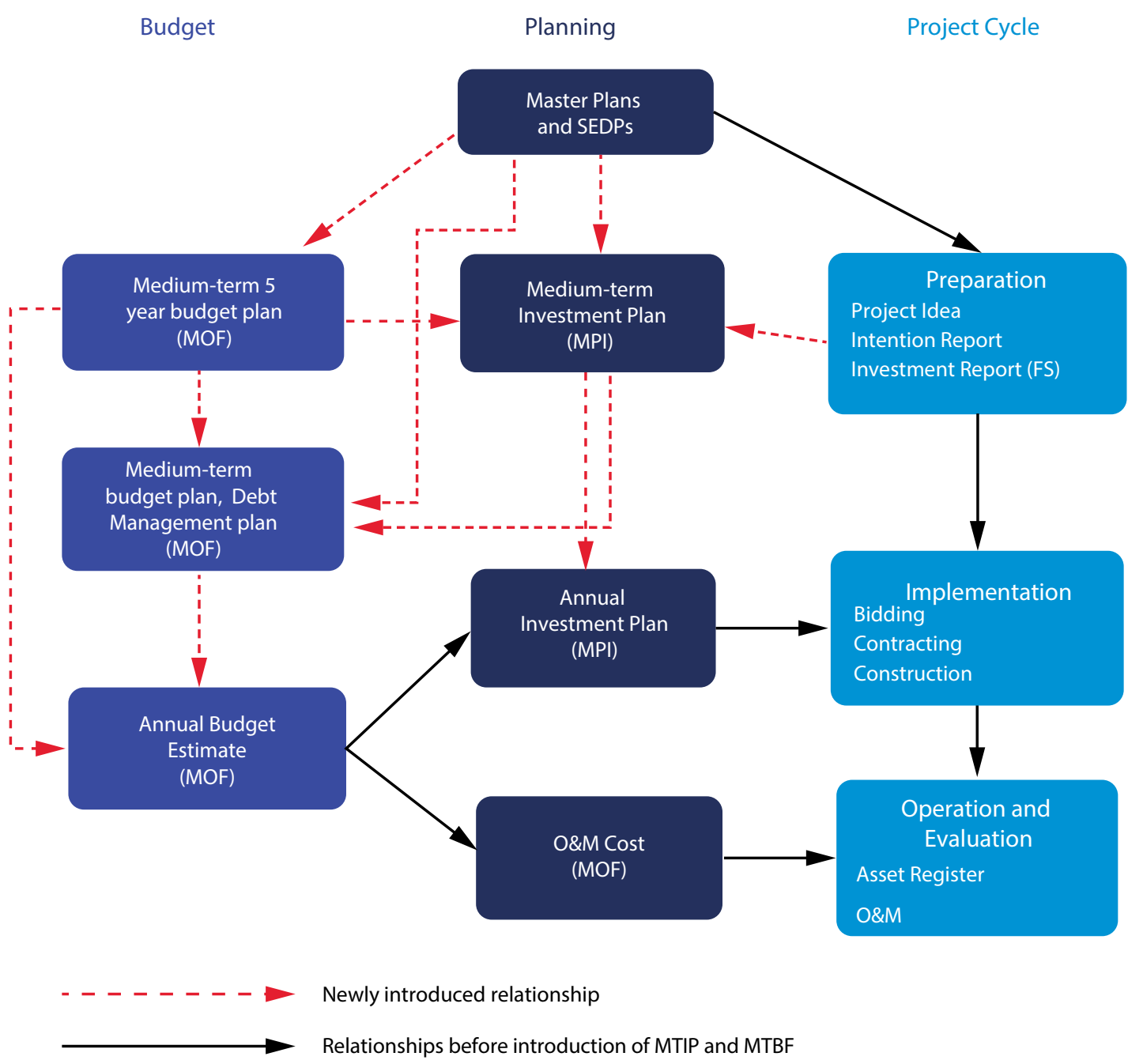

Source: Prepared by authors, based on the State Budget Law and PIL. 
38. With the PIL 2014 and the SBL 2015, Vietnam introduced a positive paradigm shift in PIM, moving from the traditional PIP approach to medium-term financial frameworks to integrate better capital and recurrent expenditures. The shift also aims to strengthen the strategic efficacy of public investment which has been downplayed because of growing decentralization. Figure 5 highlights the introduction of a number of new steps in PIM with the promulgation of the PIL and the revision of the SBL. Critical PIM-related changes include the introduction of medium-term budgeting (through the SBL, but still in the process of being operationalized), the MTIP, and the more empowered role of the central and technical ministries in the upstream appraisal steps (by the PIL and the Construction Law). Medium-term planning and budgeting were introduced to tackle the issues of time inconsistencies between long-term nature of investment and annual budget process and to improve the predictability of resources availability for investment. On the other hand, the empowerment of MOF, MPI and technical ministries, such as MOT and MOC, as regards appraisal, aims to deal with widespread negative consequences of an increasing decentralized PIM, in particular the proliferation of provincial expenditure arrears and growing concern about the technical deficiencies of on-going projects.

39. While the serious issues in relation to the inefficiency of public investment and fiscal consolidation require urgent measures, there is a need to take into consideration both the positive and negative impacts of these newly introduced changes, as well as looking at alternative policies. For example, the MOF review of repayment capability of provinces is needed from the debt management perspective because of the introduction of provincial borrowing (and on-lending) under the revised the SBL. However, the ex-ante review of every debt-financed project proposal adds another administrative step in project preparation and hence can result in notable delay. Likewise, the ex-ante review of every project proposal by MPI and MOF with respect to the affordability of funding (in case of resources from the central budget required) can lead to unnecessary delays. In the new system, the MPI and MOF are mainly concerned about the aggregate balance, so the review of every proposal may not be an optimal solution. Limiting the affordability review to largescale projects ${ }^{6}$ in combination with a periodic review of investment portfolios, improved information availability/flows, especially in relation to outstanding commitments, and enhanced accountability of investment decision-makers might be a more effective policy option. Proposals contained in the report for strengthening independent review of feasibility studies and appraisal findings for major projects will also need to pay attention to organizational efficiency and capacity constraints, and appropriate conditions must first be created to ensure added value to quality-at-entry processes from such changes.

40. The introduction of medium-term planning and budgeting in the context of decentralized PIM and dual budgeting practice imposes further critical challenges on inter-agency coordination. The challenges arose mainly from three different but inter-related aspects. First, there is limited expertise and lack of necessary conditions for the first-time preparation of MTIP. This includes detailed guidelines for preparation such as prioritization of project proposals or availability of spending baseline estimates. Decree 77, which aims to provide specific guidelines for the preparation of MTIP, only came in effect from November 2015, when spending agencies were already required to submit their first MTIP drafts. However, this aspect is only transitional by nature and is expected not to be a fundamental problem for the next MTIP. In addition, while the PIL requires the spending units to prepare the MTIP for 2016-2020, the medium-term budget plan was not being prepared during the

6 Projects from Category A and above, which are expected to have considerable fiscal implications for Vietnam or the proposing province. 
same period due to the late issuance of the SBL. Without the credible fiscal position and trajectory in the next five years, the total capital expenditure in the draft MTIP was not realistic, hence leading to a significant delay in the approval of the MTIP by the National Assembly. However, this aspect is only transitional by nature and is expected not to be a fundamental problem for the next MTIP (2021-2025) when the two medium-term plans are expected to be prepared in harmonization.

Figure 6: Timelines of MTIP Preparation

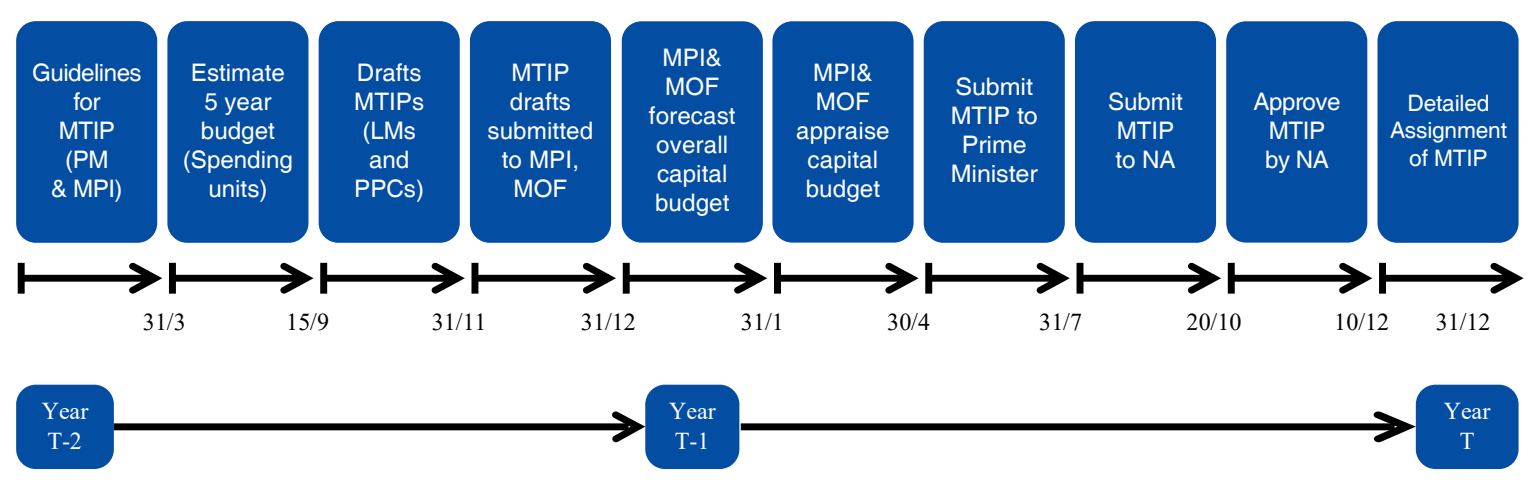

Year T: Beginning of a new MTIP

Source: Prepared by authors, based on Article 58, PIL and Decree 77/2015/ND-CP.

41. The second aspect involves a tight timelines for the preparation process in a decentralized setting. Figure 6 depicts the typical timeframe for the preparation of the SEDP, which was adopted for MTIP 2016-20. According to this ambitious timeline, planning departments only have less than 6 months to prepare the first draft of the consolidated medium-term investment plan for the provinces and line ministries. Internal reviews and appraisal are expected to take three months before the first draft is submitted to MPI and MOF. The review, appraisal, revision and resubmission of investment plans of all provinces and line ministries will take up the next six months. The MPI then has 3 months to prepare the first draft of the national MTIP, including the plan to mobilize bond financing and ODA. The entire process must also be prepared in parallel to the SEDP process, making it extremely challenging to coordinate effectively across sectors and subnational levels. As mentioned above, the specific guidelines for the preparation of MTIP 2016-20 only came into effect from November 2015, and resulted in significant delay of MTIP preparation and approval. This delay imposed further consequences for the implementation of a number of projects, since inclusion of projects in the annual plan and allocation of resources can only be considered if the project is already included in the approved MTIP.

42. The third aspect touches upon the sequence of steps in the current timelines for planning and budgeting. Overall, the budget process in Vietnam combines top-down and bottom-up elements with iteration, as is the common international practice. However, Figure 6 indicates that Vietnam starts with bottom-up activities, with requests being made by spending agencies without clear indications of financial constraints, whereas international good practice recommends starting with the top-down approach. This open-ended budget preparation practice often results in significant gap between the more ambitious aggregate "needs" as proposed by spending units and the available resources. Another sequencing anomaly for the preparation of the MTIP 2016-20 is the formulation of national allocation norms for capital expenditures - an obvious top-down element needed for entire process - Decision 40 was only issued in September 2015, one year after Prime Minister's Directive 22 guiding the SEDP preparation was issued and only after the submission of individual draft MTIPs. The order 
in which new laws have been issued (SBL in effect from 2017, whereas PIL in effect in 2015) has also imposed additional challenges to the preparation of the first set of MTIPs and aggravated the adverse consequences of the open-ended budget practice, although this is a transitional issue.

\section{Important Changes in the Upstream Part of Project Cycle}

43. In addition to the introduction of medium-term planning and budgeting, project preparation has been the prime target of recent major reforms in PIM. There is substantial evidence that poor project selection is one of the root causes of inefficiency in public investment. The emphasis on 'quality-at-entry' processes has occurred during a period of fiscal consolidation and at the same time as several mega projects with substantial time and cost overruns were brought to the public attention. The focus on the early stages of project development is in line with good international practices, since the potential pay-off from investing in improved upstream practices, leading to better project selection, can be higher because flexibility over the choice of alternative approaches is high and amendment costs are low compared to later stages, when designs are fixed and variations expensive to implement. The recently introduced reforms are looked at in depth in the relevant sections of the diagnostic assessment (Strategic Guidance, Appraisal and Independent Review); in this section, the legal and organizational aspects are reviewed.

44. Major changes were introduced in the stage where the 'project intention' is appraised and the decision on investment intention is made. Figure 7 depicts typical steps to be carried out at this stage for a budget-funded Category A project ${ }^{7}$. The changes introduced include reviews by both the MPI and the MOF (Step 4, as in Figure 7) of the affordability of the proposed project. In a nutshell, this ex ante review compares the total resource demand of the proposing agency, inclusive of the proposed project, with the estimated budget envelope for this agency ${ }^{8}$. For provinces, another MOF review is required if borrowing, including on-lending of ODA, is proposed. While the first review aims to address growing concerns about the proliferation of projects as a result of the increased delegation of core PIM functions to spending units, the second clearly reflects caution about recent steep increase in the level of public debt.

7 The ODA projects, governed by a specific Decree, will be discussed later in this section.

8 The review was already introduced with Directive 1792 in 2011, but then "upgraded" to the Public Investment Law, in effect from January 2015. 
Figure 7: Appraisal and Approval of Investment Intention Report

Project Category A

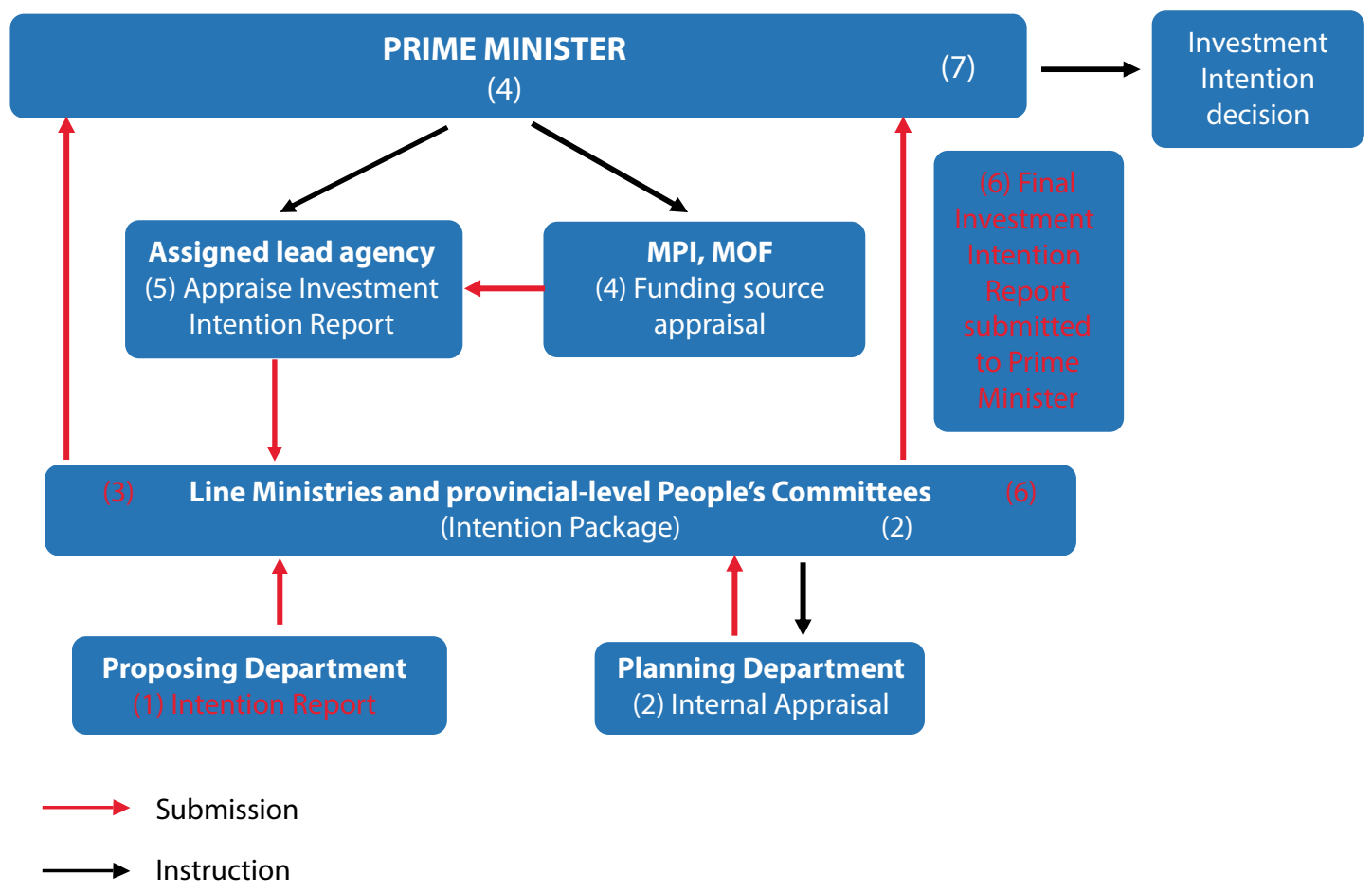

Source: Prepared by Authors, based on PIL.

45. Some element of independent review in project appraisal was also introduced with the amendment of the Construction Law in 2014. The leading role in the formal review of proposed civil construction projects is now shifted to the MOC instead of proposing sectors ${ }^{9}$. This effort to centralize the appraisal function aims to address the widespread quality issues in the preparation of construction projects. Nevertheless, it does little to address the inattention to economic efficiency in the formerly delegated appraisal mechanism, since the MOC's appraisal practice tends to emphasize technical aspects of construction, while paying inadequate attention to the extent to which economic feasibility is demonstrated in the feasibility studies of the proposed projects. This issue is addressed in more depth in Section 6 of the report where the appraisal function is assessed.

46. In addition to changes in process, recent reform efforts also aims to improve the substance of appraisal and pre-appraisal, especially at the project intention step. The Public Investment Law and its associated Decrees contain several articles that elaborate the content of the PFS or project intention report, including the specification of criteria for appraisal and prioritization. This is a major improvement to tackle the issue of poor project selection. However, effective implementation of this legal foundation requires further work to build capacity, to formulate standardized sector-specific methodologies and to establish a project database to facilitate low-cost preparation of project intention reports using quality data and information on comparable projects in this database.

9 Appraisal of sector-specific investment projects (Transport, Industry and Trade, and Agriculture and Rural Development) will still be led by the corresponding sector, rather than MOC. 
47. A review of the practical impacts of the centralization of some PIM functions, as described above - either the ex-ante review of affordability or repayment capability of each project or centralization of appraisal of the FS report - will be useful for informing further reform in PIM. The new review steps may lead to potential delay in project preparation, which must be balanced against any positive impacts on the quality of decision-making. The appraisal of project affordability is a new step, and according to the PIL, should be done within 30 days from reception of the complete submission package for a Category A project and within 15 days for Category B and C projects. Likewise, the Construction Law also sets a cap on the appraisal time for investment reports, but the additional workload and limited capacity of the existing small appraisal units in the MOC mean that unintended delays may occur. Delays can be significant, but potentially avoidable through better organization of the work flow. The good intentions of the reforms may therefore be lost because of the practical realities their implementation.

\section{Recent Changes with regards to ODA Projects}

48. ODA has long been an important source of investment financing for public projects in Vietnam. For the period 2011-15, Vietnam disbursed about US\$ 4.5 billion a year on average, accounting for almost one half of total public investment from the state budget ${ }^{10}$. Significant improvements in harmonizing the procedures for ODA and non-ODA projects have taken place during the two decades of successful management and implementation of ODA projects in Vietnam.

49. Further efforts to narrow the differences between ODA and budget-funded projects have been exerted in the last couple of years. These have been achieved through the issuance of a set of important documents, including the Public Investment Law (2014), the State Budget Law (2015), Decree No. 16 on ODA Management (2016), and Decree 52 (2017) on Management of On-lending Government's External Borrowing. The on-going revision of the Law on Public Debt Management is expected to have important implications for the ODA management as well, given the emphasis placed on the sustainability of public debt and the transition towards less concessional ODA sources.

50. The inclusion of ODA in the budget ${ }^{11}$ is arguably the most important change and has considerable implications for ODA projects. Merging ODA resources with the budget follows good international practices and is expected to strengthen the debt management system, as well as contributing to enhancing PIM by imposing stronger budget discipline. Together with the inclusion in the budget of government bond issuance for financing infrastructure, this change helps reduce the exposure of projects to variable or multiple regulatory frameworks according to financing source or sources.

51. Nevertheless, important differences between procedures for ODA and non-ODA projects still remain. Compared to a non-ODA project, additional formal decisions are needed to initiate an ODA project, where proposing line ministries or provinces need to work closely with DPs and then have the project appraised by an MPI-led process. This step, depicted in Figure 8, concludes with the MPI's informing the proposing agency about the PM's approval so that the next step, the preparation of the investment intention, can begin. The appraisal involves consultation with several agencies such as MOF, Office of Government (OOG) and the State Bank of Vietnam (SBV) and consequently needs more

10 Framework for Mobilization, Management and Usage of ODA and Concessional Loans for 2016-20, issued with Prime Minister Decision 251, in February, 2016.

11 Public Investment Law 2014, Article 4. 
time than "ordinary" projects. Another ODA-specific step relates to the signing and effectiveness of project agreement, which involves the endorsement of the President after consultation with relevant Committees of the National Assembly. Finally, the inclusion of an ODA project in the MTIP only takes place after the signing of the loan agreement instead of after the approval of the investment intention in the case of non-ODA projects. As a result, it normally takes ODA project/program at least one to two years after the project/program is approved by donor for the implementing agencies to initiate the project implementation, including procurement of project activities. ${ }^{12}$

\section{Figure 8: Steps in ODA Project Concept Idea}

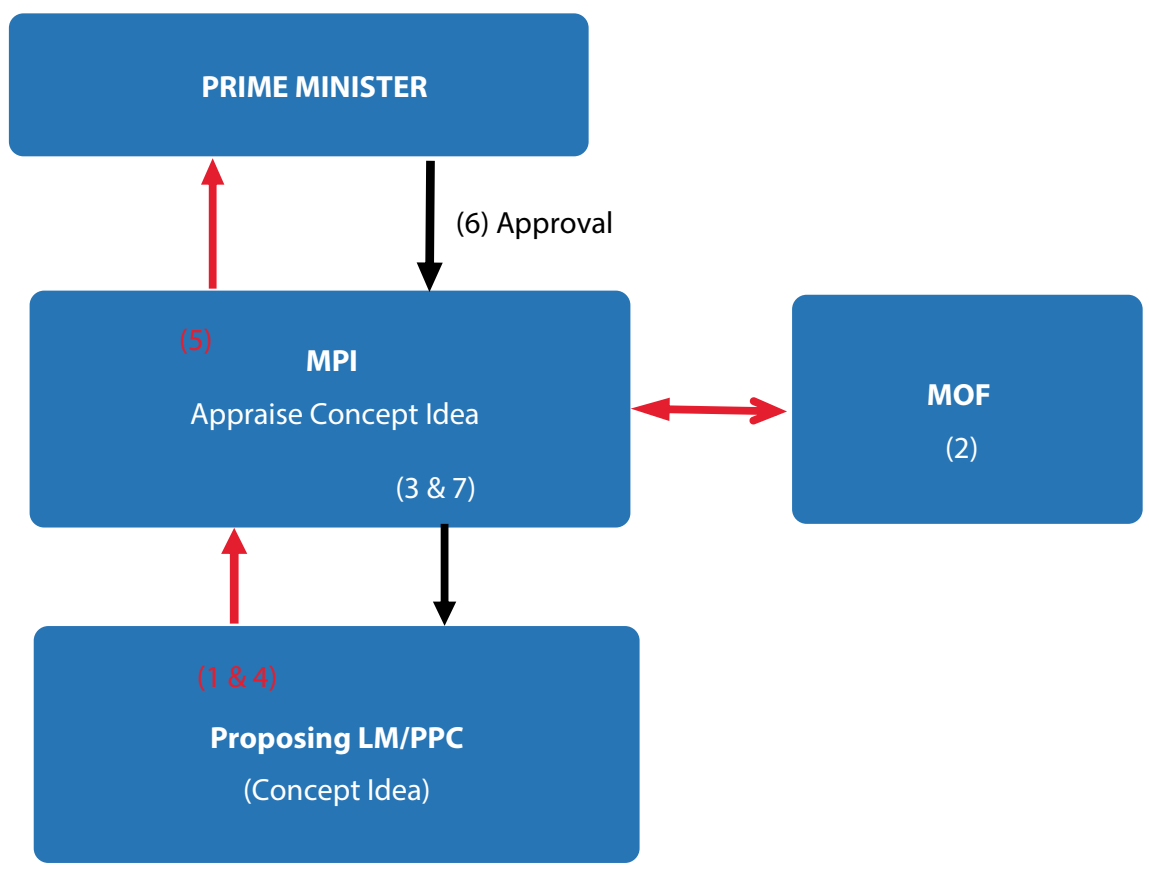

Source: Prepared by Authors, based on the PIL and Decree 16/2016. The appraisal of an ODA investment intention follows similar procedures depicted in Figure 7.

\section{The inclusion of ODA in the state budget in a period of significant changes in the legal framework for budgeting and PIM may also lead to unintended but transitional consequences. For example, the Law on State Budget 2015 and the Resolution on the State Budget Estimates of the National Assembly require that expenditures must be estimated and the execution and disbursement must be in line with the assigned capital plan. According to this provision, starting from 2016, ODA programs and projects and preferential loans will no longer be disbursed according to implementation progress and the commitments made in related project agreements with donors, as before. Many ODA projects have been experiencing delay due to this newly introduced disbursement process.}

12 There are additional differences between ODA and non-ODA projects when it comes to arrangements for specific types of projects. For example, a multi-province non-ODA project belongs to the management of the relevant central line ministry, whereas each participating province in a multi-province ODA project will have to go through the same preparation procedures. The issues associated with the lack of coordination in preparation can, however, be compensated by the advantages of higher level of authority in implementation. Another difference involves the financing structure of an ODA project that can involve both on-granting and on-lending, hence adds further complexity to the project preparation and management. 
53. While the introduction of fixed expenditure plans is inherently a transitional problem for the implementation of on-going ODA projects, the required alignment of DPs' portfolios to Vietnam's MTIP is a critical challenge for the longer term. The challenge of having timely signing of the ODA loan agreements for the inclusion of projects in a fixed five-year MTIP is not straightforward, given the notable differences between the planning practices of Development Partners and Vietnam. The required budgeting approach which begins with a clear identification of financial constraints will be hard to implement without good indications of ODA commitments for the five-year planning period. The lack of clarity in the procedures for the amendment of the fixed five-year MTIP only adds more complexity to the process. In addition, the tight timelines for the preparation of the MTIP, as discussed above, represent another set of issues for the alignment of the ODA process into Vietnam's mainstream planning and budgeting process. 



\section{Strategic Guidance and First-Level Screening}

\section{What Does Good Practice Look Like?}

54. There are four main components of good practice in strategic guidance and first-level screening for public investment projects:

- A published national development strategy or vision with unambiguous authority.

- Clarity of strategic objectives in terms of project outputs and outcomes.

- Consideration of alternative approaches to achieving objectives.

- Centralized approval by planning or finance ministry for further developing proposals for major projects.

55. The minimum requirement is for a high-level and authoritative statement of the government's economic and social strategies. This is important so that investment planning can be aligned with medium to long-term national economic and social priorities. The national strategic framework should ideally be supported by more detailed, operational and annually-updated sector planning documents to guide identification of new project ideas and prioritisation of capital spending decisions.

56. Projects should then be initiated through a formal process that involves preparation of a profile of the project idea (or 'project concept note') situating any new project idea within the strategic context. In particular, the profile should indicate the reasons why the project is being undertaken, identify any alternatives that might warrant further analysis in the feasibility study, and set out the expected outputs and outcomes. Stakeholder (including the ultimate beneficiaries) involvement during the development of the project concept is an important feature of good practice models. Failure to do this is often cause of problems or failure in project implementation and operation.

57. The project profile needs to be screened by higher level authorities for policy relevance, among other things, leading to an approval to proceed with project preparation and appraisal. Major projects should be screened by the planning or finance ministries. This process is the first, and perhaps the most important, line of defence against poorly conceived investment proposals which, if not stopped, risk gaining 'planning momentum' and ending up wasting public money.

\section{Assessment of Strategic Guidance for Public Investment in Vietnam}

There is no shortage of strategic guidance for public investment in Vietnam; but there is considerable room for quality improvement in terms of coherence and realism.

58. Vietnam has what on the surface seems to be a well-developed strategic planning system, with a strong public investment orientation, where the strategies and plans that emerge are approved at the highest levels. The Government's long-term development strategy is based on a series of 10-year socio-economic development strategies (SEDS), the latest covering the period 20112020. The SEDS is made operational through five-year socio-economic development plans (SEDPs), which elaborate the objectives of the SEDS and identify the specific measures and resources required 
for its implementation. The current SEDP covers the period 2016-2020 and was approved in April 2016. As well as the national SEDP, Vietnam has 63 provinces and each province prepares its own SEDP. Action plans and annual plans are developed for implementation of the SEDPs.

59. The current 10-year Socio-Economic Development Strategy has an explicit focus on infrastructure. The overarching aim is to lay the foundations for a modern, industrialised society by 2020. It defines three 'break-though' areas for achieving this aim: i) promoting human resources/skills development (particularly skills for modern industry and innovation); ii) improving market institutions; and iii) infrastructure development ${ }^{13}$. In relation to the last area, one of the explicit goals of the SEDS is to develop infrastructure quickly, especially transport infrastructure ${ }^{14}$.

60. The SEDS and SEDPs provide a framework and direction for ministries and province departments to develop more detailed plans for their sectors and sub-sectors, which are usually approved by the executive branch of government. It is notable that the high-level sector plans also tend to have a longer-term vision that extends beyond the planning period, potentially extending their usefulness for developing a project pipeline. Taking the example of the transport sector, longterm masterplans and medium-term development plans are developed. At the national level, the highest planning instrument is the 'Transport Development Strategy up to 2020 with a Vision toward $2030^{\prime 15}$. Beneath this are sub-sector strategies, such as the 'Road Transportation Development Plan by 2020 and Vision toward 2030' and detailed master plans, such as the 'Detailed Masterplan for the North-South Expressway- Eastern Side - 2010-2023'. This structure is mirrored at the province level. In the energy sector, taking another example, the highest level planning instrument is the 'National Energy Development Strategy to 2020, with an Outlook to 2050'. Below this, more detailed plans exist, such as the 'Revision of the Power Development Plan for 2011-2020, with a vision to 2030'16.

61. While apparently well organised, the strategic planning system has weaknesses in terms of the realism of the individual strategies and plans, and the coherence between the different levels. Commenting on SEDP 2011-15, the World Bank expressed the following concerns:

The SEDP could have been clearer in two areas: i) the importance of identifying explicit trade-offs in priorities and proposed policy measures, particularly in the light of real resource constraints; and ii) the need for greater coherence, integration and linkage between the national SEDP, provincial SEDPs, and sector plans being developed by ministries. ${ }^{17}$

While steps to address these concerns have been taken in elaborating SEDP 2016-20, they remain relevant.

62. Plans tend to be over-ambitious, particularly in relation to the financial resources available for their implementation. SEDP 2016-20 acknowledges the slow progress on certain reform priorities in the previous planning period and emphasizes the need to accelerate reforms over the current planning period in order to achieve targets ${ }^{18}$. Some of the slow progress may indeed have arisen from implementation delays, but over-ambitious targets, set with insufficient attention to financial resource constraints, are very likely to have been part of the problem. This is a function of a planning system with teleological origins (as opposed to a more demand-driven, indicative system).

\footnotetext{
http://www.worldbank.org/en/country/vietnam/overview

World Bank Group's Country Partnership Strategy for Vietnam (FY12-16)

Another important infrastructure sector, energy, has a similar long-term instrument,

This is a revised version of the Power Master Plan No. VII.

World Bank Group's Country Partnership Strategy for Vietnam (FY12-16)

8 http://www.worldbank.org/en/country/vietnam/overview
} 
63. The coherence of long-term sector plans with the higher-level planning instruments is an issue. This has been recognised and attempts to align better the different instruments have been made. The original Transport Development Strategy was prepared before SEDS 2011-20, but was adjusted in 2009 to reflect the SEDS better. Similarly, the latest Power Development Plan, is a revision of an earlier plan. Sometimes, differences in timing reflect the realities of implementation, which are not necessarily the same as formal planning horizons, as in the above example of the North-South Expressway, where the master plan straddles two SEDSs. In spite of steps taken to improve coherence, there remain weaknesses.

64. The limited linkage between the formulation of investment programs set out in SEDPs and the budgeting process makes the achievement of these, often ambitious, programs rather challenging. SEDPs are accompanied by an investment program, listing the projects to be implemented during the planning period. In theory, this should be a good starting point for initiating and developing projects. However, the lists are compiled from 'bottom-up' requests from sectors and assembled with limited regard to the likely availability of financing and no firm prioritisation. In addition, new projects can enter the investment program during plan implementation with relative ease, thus limiting its usefulness as a filter for strategic relevance. The lists of projects included in the SEDPs are often derived from 10-year master plans, which themselves have been derived from a bottom-up process and are subject to revision with relative ease. In addition, the current master plans cover the period 2011-2020 and were prepared before the implementation of the new Public Investment Law. This means that projects within them will not have been subject to the more rigorous first-level screening described below. Box 2 provides some background on these weaknesses at the province level.

65. Planning in Vietnam is more complex than is captured in the preceding discussion, extending far beyond the higher level instruments, the SEDS and the SEDP. In practice, there is a complex and overlapping system of more than 20,000 long-term master plans, including four main types: (1) Social and economic Master Plans at regional, provincial and district levels; (2) Land-use Strategies and Master Plans at national, provincial an district levels; (3) Construction Master Plans at provincial and district levels and (4) Sector Master Plans covering the fields such as transportation, industry, commerce, agriculture, economic zones, border gate economic zones or power plants. These plans also suffer from problems of financial realism and lack of coherence between the different types, and they generate a huge list of planned and approved investment projects which bear no relation to any budget constraints. Recommendations on simplifying and streamlining the planning system are beyond the scope of this study; nevertheless a requirement for some sort of overarching planning instrument to cut through the existing complexity and steer investment towards major priority infrastructure projects in a fiscally sustainable way does strongly suggest itself.

66. Strategic planning in Vietnam has a supply-driven, investment bias, whereby the creation of new fixed assets is often emphasised over the identification and achievement of wider development objectives through improved public service delivery. The planning system is regulated by the Ministry of Planning and Investment (MPI), but the 'investment-bias' reflects institutionalised attachment to an economic development model that emphasises investment over other drivers of growth ${ }^{19}$, together with a political system that tends to reward creation (or commencement) of new assets, more than more mundane improvements in public service delivery.

19 Statistical analysis using growth accounting models tend to find that capital investment is not the only, or even the most important, explanatory variable for economic growth. 
The highly decentralised approach to public capital investment planning in Vietnam also means that the degree to which a central body like the MPI can influence planning choices is very limited.

\section{Attitudes to planning may be changing, but any changes in thinking are slow to be reflected} in the planning system itself. Investment for investment's sake has been to the detriment of a clearer articulation of the higher-level development objectives (outcomes) to which investment is expected to contribute through improved service delivery (quantity and quality of outputs). In consequence, insufficient attention is given to alternative, less capital-intensive development strategies, and to maintaining the performance of the existing stock of fixed assets ${ }^{20}$. In addition, the focus on supply underplays the importance of confirming demand for services from newly created assets, running the risk of expensive 'white elephant' projects, for which there is little or no demand.

\section{Box 2: Planning and Budgeting for Capital Investment at Local Level}

Every five years, the PPC has to develop the 5-year socio-economic development plan (SEDP), which is considered the medium-term strategic plan of the province. The 5-year plan is discussed and approved in the last provincial party congress and is the basis for issuing the 5-year resolution of the Provincial Party Bureau, which is seen as 'must-do' list of targets/indicators to be achieved over the next 5 years. The most recent 5-year planning period is 2011-2015, which now also coincides with the budget stability period.

The 5-year SEDP includes key performance indicators, tasks and solutions to achieve the set objectives, together with a 'shopping list' of capital investment projects for funding over the five years. These are then intended to be translated into subsequent annual SEDPs.

In principle, only projects appearing in this list can be reviewed for actual funding allocation in the course of the five years. However, due to poor planning skills and information, weak fiscal discipline and strong discretionary interference from the political level, this list is subject to change over the years and many projects can and do jump the fence ('fast processing') for approval. This is a key factor leading to substantial capital funding shortages and project delays, which are typical in transport sector (since this is among the most capital intensive sectors in province). To fix the problem, Directive No. 1792/20111 on strengthening investment management from State budget capital and government bond capital was issued by the Prime Minister in 2011. The directive required that only investment projects which can show evidence of reliable funding commitment should be approved. Although not intended as a policy reversal in the decentralization of planning - it was introduced to ensure fiscal discipline - Directive 1792 has, in fact, reduced local level decision making on public investment programming.

Source:'Technical Assistance for Medium-Term Expenditure Frameworks for Provincial Road Management: Phase 1'World Bank, 2015.

Changes introduced through the Public Investment Law have improved the strategic planning basis for PIM, but there remain some shortcomings.

68. The 2015 Public Investment Law (PIL) has changed the environment for strategic planning of public investment, with the introduction of a new instrument, the medium-term plan for public investment (MTIP). Like the SEDP, the MTIP is a static plan prepared every five years by central government entities and local authorities, with the timetable for doing so laid out in the PIL. The MTIP should be based on the SEDS and on the relevant SEDP and sector plans, and should be supportive of the achievement of the targets of these plans. The contents of the plan and the criteria for selecting

20 This was the broad conclusion of a recent World Bank report on the planning and financing of road maintenance at the province level: 'Technical Assistance for Medium-Term Expenditure Frameworks for Provincial Road Management: Phase 1' World Bank, 2015. 
programs and projects for potential financing ${ }^{21}$ are set out in the law. The latter include a requirement that sufficient financial resources are allocated to finish projects on schedule. Most importantly, the MTIP must be affordable:

Such plans must conform to the ability to balance the capital budget for public investment and attract the capital for other economic sectors; secure the government debt. ${ }^{22}$

69. Another feature of MTIPs is a requirement for the projects included to have passed the firstlevel screening process set out in the PIL, as described below ${ }^{23}$. First-level screening is potentially good for assuring the strategic relevance and affordability of the plan, but means that all of the project identification effort is squeezed into a narrow period during five-yearly preparation of the plan. While it seems like a good idea for the most significant projects to be included explicitly in strategic plans, more flexibility with respect to other projects may be useful, allowing specific projects to be identified and pass first-level screening during implementation of the plan.

70. While the MTIP should, in principle, strengthen the strategic orientation of public investment, it suffers from the inherent inconveniences of a static five-year planning period. Static plans introduce inflexibility in a potentially dynamic environment, where change is almost inevitable, particularly with respect to the availability of financial resources. It also makes it difficult to plan for the many major projects that are likely to bridge the different planning periods. Although adjustment of plans is foreseen, the process is reactive and potentially cumbersome. One way around this could be to adopt a rolling infrastructure plan, either at the national level (see Box 3 for the Australian case) or for key infrastructure sectors like transport as is done in Sweden and Norway ${ }^{24}$. A rolling infrastructure plan can take account of the changing macro-fiscal environment and new strategic priorities, as well as encompassing nationally important projects the planning and implementation of which may take significantly longer than five years.

71. The 2015 SBL requires the preparation of a medium-term (three- and five-year) financial plan, but there is more work to be done in developing this as a viable financial framework for medium-term planning. While it is important that a medium-term financial framework is prepared as a constraint on the elaboration of unrealistic SEDPs and linked MTIPs, the quality and consistency of this framework still needs to be worked on before it represents a credible restraints to the development of unaffordable plans. The fact that the five-year MTBF is a fixed framework, and not rolled over, means that it will tend to become increasingly less credible as the plan period advances.

72. Because of earlier weaknesses, the first MTIPs prepared under the PIL have had limited scope for financing new projects and the preparation of new projects. As will be discussed further in the section on Selection and Budgeting, weak capital budgeting has resulted in a backlog of ongoing projects, many of which have received insufficient finance for efficient implementation and some of which are effectively stalled. Once these had been planned for properly in the MTIP, there would have been limited scope for affording new projects. The implication of this, apart from a the need for a more disciplined budget process, is that there is a need for a clean-up and reprioritization of the portfolio of on-going projects before the MTIPs can work effectively. In the current context,

21 The MTIP is indicative and does not represent a funding commitment.

22 Article 51.2 of the Public Investment Law

23 Article 55 of the PIL sets out the eligibility criteria for programs and projects to be allocated funding through the MTIP. The first criterion is, 'The competent authorities have decided the investment policies'.

24 Sweden and Norway have long-term (10 year) rolling (every 4 years) infrastructure plans, for transport infrastructure only. 
when developing the MTIPs, the underlying incentives may well have been to continue underfunding on-going projects in order to make space for the new projects required to achieve the SEDPs. In this context, the MTIPs will tend to perpetuate the existing weaknesses, without supporting initiatives.

73. Preparation of strategically coherent MTIPs requires timely preparation of the SEDPs, which was not the case for the current SEDP. The SEDP 2016-2020 was approved in April 2016, which would have been too late to be taken into account in preparing MTIPs, if the timetable set out in the PIL were to have been followed. This emphasises the need to ensure good sequencing and adequate resourcing of planning activities across the different layers of Vietnam's complex planning system, if strategic plans are to provide timely and operational guidance for initiating projects.

\section{Box 3: Australian Infrastructure Plan 2016-2031}

In 2014, Infrastructure Australia, an independent statutory body with a mandate to prioritise and progress nationally significant infrastructure, was tasked with preparing a rolling, long-term national infrastructure plan.

This Australian Infrastructure Plan 2016-2031 was developed through a collaborative 18-month process of research and consultation. To underpin the planning process, two infrastructure audits were performed, the Northern Australia Audit and the Australian Infrastructure Audit. The latter represents the nation's first comprehensive examination of infrastructure across the energy, telecommunications, water and transport sectors. Together, the two audits provide the primary evidence base for the plan. They set out the case for substantially enhancing the quality, capacity and efficiency of infrastructure and overhauling the way infrastructure is planned, funded, constructed, operated and maintained.

Following release of these audits, Infrastructure Australia received more than 100 formal submissions from jurisdictions, a wide range of industry associations, public interest groups, local government bodies and individuals. More than 500 stakeholders were consulted in every state and territory, and worked closely with representatives from all levels of government, as well as businesses, industry, peak bodies and the wider community.

The plan lays out a comprehensive package of reforms focused on improving the way Australia invests in, delivers and uses its infrastructure. The aim is to focus on extracting the greatest value from existing infrastructure, while sustainably funding new investments to deliver better services for all Australians. The reforms in the plan are intended to be guided by four headline aspirations:

- Productive cities, productive regions;

- Efficient infrastructure markets;

- Sustainable and equitable infrastructure; and

- Better decisions and better delivery.

The result is a long-term strategy that aims to lay the foundation for a more productive Australia over the coming 15 years and beyond.

Sources: 'Australian Infrastructure Plan', Infrastructure Australia, 2016 and http://infrastructureaustralia.gov.au/

The Public Investment Law has introduced some important improvements to first-level screening, which align in many ways with good practice models, but further improvements are required in relation to: i) the decisive status subsequently given to the preliminary cost estimates; ii) the limited scope of the screening role allocated to the MPl; and iii) the fragmentation of responsibilities according to the nature of the project and the funding source. 
74. Prior to the PIL, there was no formal requirement to prepare project profiles and no systematic screening of project concepts to reach a decision on whether to proceed to preparation. The project sample indicates that a project profile was prepared in many cases ${ }^{25}$, but not on a systematic basis or to a consistent format. Only projects of national importance were required to have a pre-feasibility study. Appearance in an approved plan was sufficient for Group A projects and there was no requirement for a pre-feasibility study for Group B projects, provided the authorising agent agreed. As evidence from the sample shows, the majority of projects developed prior to the enactment of the PIL in 2014, were not systematically screened at identification by an external party. The probable result would have been few projects stopped at identification stage on the basis of a weak strategic case or poorly developed rationale. This also means that there were limited opportunities for the central planning and financial agencies - the MPI and MOF - to review concepts for major projects before decisions were taken by central entities and local authorities.

75. The PIL has introduced an important first stage in the PIM system, whereby a formal decision is now taken on whether to proceed to preparation and appraisal, on the basis of an early analysis of the project concept. This brings Vietnam into line with international good practice. The decision on investment policies (or intentions), as it is known, is taken on the basis of standardised explanatory material, the complexity of which varies by project size. For Nationally Important projects and Group-A projects, a Pre-Feasibility Study (PFS) report is required, while for Group-B and -C projects, a less sophisticated 'Report on Investment Intentions' (ROII) is required. Both reports are defined in the same way as'... explanatory material for preliminary study contents about the significance, feasibility and efficiency...' of projects, but their detailed contents and depth of analysis differ significantly.

76. The Report on Investment Intentions has the features of a project profile as envisaged in the good practice framework. The broad contents are set out in the PIL ${ }^{26}$ and, along with a basic description of the project ${ }^{27}$, include preliminary assessments of social and environmental impacts and socio-economic effectiveness, as well as a preliminary estimate of total investment costs and of future operating costs. While the rationale for the project must be explored, there is more of an emphasis on demonstrating the affordability ${ }^{28}$ of the project. This derives from the increased fiscal discipline intended through the medium-term fiscal plan and the newly mandated role of the MPI and MOF in policing affordability considerations. Overall, the contents of the ROII compare well to the typical examples of concept notes given in Box 4 for Colombia and South Korea. Project alternatives, demand and risk are not covered explicitly, but neither are they always dealt with separately in other countries.

\section{Moving straight to the level of analysis involved in a PFS, without making an initial decision} to proceed based on a simpler project profile, is not typical of good practice countries. The contents of the PFS as defined in the PIL conform in broad terms with international standards for this kind of study. It is common practice to undertake a PFS as part of the appraisal of major projects, but this is usually preceded by a less sophisticated presentation of the case for the project, to be sure that the project merits in-depth analysis. Taking South Korea as one example, examination by the finance ministry of the project profile described in Box 4, leads to a decision to carry out a preliminary feasibility study of major projects, which is then followed by a feasibility study. A PFS is usually the same in content and analytical approach as a feasibility study; the difference is that the PFS is usually

25 Seven out of 10 transport projects had some form of project profile prepared at inception and all education projects.

26 Article 36 of Public Investment Law.

27 'Objectives, size, site location and coverage...'

28 'Estimate of total investment, capital structure, the balancing capability of public investment portfolio, mobilisation of different funds and resources for the project execution.' 
based on readily available, secondary data and an outline design, rather than primary research and a preliminary design, as required for a feasibility study. From discussions and the findings of the sample survey of projects, this is also the understanding and intended practice of a PFS in Vietnam ${ }^{29}$.

78. A PFS should not be the sole basis for reaching a decision on whether a project represents a good use of public money. Although a Feasibility Study (FS) is undertaken in Vietnam, the investment approval decision is taken on the basis of the PFS (or ROII), and the increased analytical depth and reliability that should, in theory, be provided through the preparation and appraisal of a feasibility study does not then effect this decision. Once the PFS (or ROII) is approved, the budget that approved at this stage gets locked into the MTIP and there is limited scope for changing it at the FS stage. In practice therefore, the FS is more of a design study and there is, effectively, no decisive appraisal decision after the PFS, thus removing one of the important quality control filters expected of a robust system, as is explored more in the next section.

79. Beginning at the concept stage and then throughout the PIM system, the PIL makes a distinction between the analytical requirements for non-construction projects and construction projects, leading to a fragmented and potentially inconsistent approach. As indicated in the $\mathrm{PIL}^{30}$, construction projects are subject to a different legal framework when it comes to the contents of the PFS. The general table of contents established in the PIL, which applies for non-construction projects, is longer and implies more detailed analysis than for construction projects, as set out in the Construction Law (2014). There is no logical reason for a difference in the level of detail or content between the two types of projects which, provided they meet the definition of public capital investment, would be treated the same in most other countries' systems. It could well be that the contents in the Construction Law are just expressed more succinctly and that same depth of analysis is expected for both types of project, but this is by no means clear at this level in the legal and regulatory framework. Even if this is intended to be the case, the current dichotomy leaves too much room for ambiguity in interpretation and for inconsistency in application, and a fully unified legal framework for public should be considered.

80. There is no comprehensive guidance to assist those formulating reports for a decision on investment intentions. The summary contents for the ROIl and the PFS, as set out in the PIL (and its $g$ legal documents), are a good starting point, but without detailed methodological guidance it seems likely to be difficult to achieve a consistent quality of submission, because of differences in interpretation and in understanding of the analytical and design requirements. As already suggested, without further guidance on what is expected and on how to conduct the analysis, the contents of the PFS could simply be interpreted as a more elaborate version of the ROIl, whereas something closer to a stripped down feasibility study is probably intended. Even the ROII requires further methodological guidance that goes beyond the usual scope of primary and secondary legislation to put it into practice.

81. The MPI and MOF are given narrow responsibilities in relation to advising on the decision on investment intentions, which are largely confined to assessing the investment portfolio and portfolio balancing capability' of project proposals. A clear allocation of roles and responsibilities at this stage is established in the PIL according to level of government and project characteristics. For

29 Although the contents set out in the PIL could, without further methodological guidance, also be interpreted as a more elaborate form of ROII (project profile). How to interpret 'Preliminary estimate of effectiveness in socio-economic development' is one critical area open to interpretation and requiring guidance. A PFS would require social cost-benefit analysis to be performed (where applicable) to provide such an estimate.

30 Article 25 of Public Investment Law. 
Nationally Important Projects, the National Assembly is the ultimate decision-maker on investment intentions, based on a proposal from the Government. The MPI has no direct role, but chairs the State Council responsible for appraisal of the PFS report. In the case of Group A projects, it is the Prime Minister who decides on investment intentions. An Inter-sectoral Council carries out an independent appraisal of the PFS and the MPI, in cooperation with the MOF, assesses the affordability of the proposal. For Group B and C projects, appraisal is confined to an 'internal' appraisal at the level of the responsible central body or local authority, and the MPI, with the MOF, assesses affordability.

\section{The Investment Appraisal and Supervision Department within the MPI is the focal point for} collecting comments on a PFS submission. Although its assessment of Group A projects is restricted by the PIL to affordability issues, the MPI does take a wider view, examining necessity and strategic relevance in consultation with specialised sector departments, like the Science, Education, Natural Resource and Environment Department and the Infrastructure and Urban Development Department. The standing of assessments that go beyond affordability issues is limited, however, and the impact on which projects go forward to appraisal correspondingly low. The sectoral departments also have a role in commenting on the necessity and strategic relevance of Group B and C projects promoted by central agencies, but this is done independently of the Appraisal and Monitoring Department and their assessments are non-binding.

83. Responsibilities for different components of first-level screening are fragmented. This can be illustrated by the roles assigned to different agencies for reviewing Group A projects. While the MPI and MOF are jointly responsible for assessing affordability, "technical" line ministries are involved in assessing the cost estimates and preliminary design aspects of projects. The lead central agency or local authority has responsibility for confirming the 'necessity' for the project. Fragmentation can be managed if there is good coordination, but this is not always the case: for example, the assessment of cost estimates may not always precede the MPI/MOF assessment of affordability, as would be logical. Even within the MPI there is some fragmentation of responsibilities, with the Department of External Economics being responsible for assessing ODA-funded projects (Group B and below), which it does largely independently of the Appraisal and Monitoring Department.

84. When the MPI has assessed the affordability of a Group A project, there is no further opportunity to review and comment on the revised PFS. The MPI submits its report on the PFS to the Inter-sectoral Council or the appraisal leading agency, which then sends it to the responsible central agency or local authority. The latter completes the PFS, taking account of the review by the $\mathrm{MPI}^{31}$, and submits directly to the Prime Minister without going through the MPI again. In some case (around one in ten), the Prime Minister requests the MPI to review the new version of the PFS, but this is not systematic. There is justifiable concern about this within the MPI (and the MOF, with which the assessment of affordability is coordinated), because the MPI has no way of verifying if its advice has been acted upon, thus diminishing the status of its assessment. The limited role of both MPI and MOF in reviewing investment costs after the investment intention decision stage explains why the emphasis of the review of investment intentions is often on investment cost, rather than on strategic relevance and rationale, which would generally be the case elsewhere.

31 Article 23.4 of the Public Investment Law. 
85. The PIL spells out the general criteria for the decision on investment intentions for a project, made on the basis of the PFS and the ROII, but these are not spelled out in sufficient detail in supporting regulations. The criteria in the $\mathrm{PIL}^{32}$ are, for the most part, a good starting point for firstlevel screening, but are difficult to operationalise without further detail and supporting guidance on their application. Discussions with participants indicate that more detailed criteria for internal use have also not been developed. The final criteria relating to prioritising public-private partnerships is premature and, while PPP potential, might be flagged at this stage, this should not be a reason for accepting or rejecting a project at this point in project development without further analysis of the economic feasibility.

86. Project costs are fixed too early in the project cycle, before any substantive design work has been done, leading to potential 'gaming' of the system, and avoidable adjustments to costs and re-scoping during implementation. The cost estimate that is included in the ROII and PFS, and approved in the investment intentions decision subsequently become part of the investment decision, the legal approval of the project. The estimate for procurement purposes comes from the investment decision and represents a ceiling for bids. There are several possible responses to this. The first response could be that the costs included by project promoters/developers in the ROII and PFS will be exaggerated to make sure that any possible cost increase is allowed for: this may lead to unused budgets or 'gold-plating' of projects to use up allocations. The second response is to adjust outputs or the quality of outputs downwards in later stages of the project cycle in order to remain within the ceiling fixed in the investment decision: this might make the project's benefits less easy to achieve. The third response is to adjust costs after the investment decision, but this is subject to a cumbersome approval process, that most spending agencies would prefer to avoid. In other systems, the fixing of project costs is done later through a more gradual process. In such systems, costs at the project concept stage are just indicative - for expenditure programming purposes - and become firmer when a feasibility study, based on a preliminary design, is completed and approval 'in principle' is given. Costs are not finally tied down for final approval and budgeting purposes until the results of the procurement process are confirmed, and the final cost may be above or below the approved feasibility estimates ${ }^{33}$.

87. The quality of PFS/ROII submissions is very uneven and this needs to be addressed if implementation of the new PIM framework is to be successful. The PIL is a new instrument and initial challenges in applying it are to be expected; nevertheless, it is clear that there is still considerable work to be done to raise the quality of the first-level submissions to an acceptable standard. Weaknesses include poor justifications of the necessity projects and, for PFSs, largely qualitative assessments of benefits in sectors, like transport, where valuation is usually possible. The $\mathrm{MPI}$ is best placed to take the lead in promoting better practices, firstly through developing and issuing methodological guidance, and then through training in the application of this guidance.

32 Article 18 of the PIL. The criteria can be summarised as: strategic relevance; avoidance of duplication; affordability; creditworthiness; social profitability; and PPP potential.

33 If costs are significantly above feasibility estimates, there may be a requirement to re-work the feasibility study with the new costs to ensure that the project remains socially profitable. 


\section{Box 4: Examples of Formats for Project Profiles}

Although the detail varies between countries, information requirements for project concept notes are generally similar, covering the nature of the problem to be addressed, the urgency of the need, the rationale for the proposed solution, and the links with national and sector strategies. Colombia, and South Korea provide two examples formats for a project concept notes, with the former probably representing the minimum requirement.

\section{Colombia (Identification Stage):}

- Identification of the current situation and the expected future situation

- Diagnosis of the problem or need to be addressed, including identification of target population and their social and economic characteristics

- Rationale for the project solution and its objectives

- Identification of alternative solutions

Source: 'Manual Metodologico General, para la Identificación, Preparación y Evaluación de Proyectos', National Planning Department, Colombia 2004.

\section{South Korea (Written Request for Preliminary Feasibility Study):}

- Draft project plan, including:

- Project purpose

- Related developments and scale of the project

- Estimated total project costs

- Implementation system

○ Financing method

- Anticipated benefits

- Need for project implementation

- Adequacy of likely central government subsidy [affordability]

- Amount and financing method of necessary resources

- Potential contribution to balanced regional development ('need for technological development' in the case of an R\&D project)

- Risks associated with project implementation and mitigation measures.

Source: 'Operational Guidelines for the Preliminary Feasibility Study', Ministry of Strategy \& Finance, South Korea, 2009. 


\section{Summary Assessment of Strategic Guidance and First-level Screening}

Key Functionality
Projects are subject
to actionable
strategic guidance
and project
proposals are
subject to first-
level screening
in relation to this
guidance

2. Centralised approval by planning or finance ministry for developing proposals. 1. Published development
strategy or vision with
unambiguous authority.

\section{Current Arrangements in Vietnam}

Vietnam has a well-established planning system providing strong strategic guidance for identifying new project concepts. The Medium-Term Investment Plans introduced through the PIL add another layer to the planning system and further ensure the strategic relevance of project proposals. The realism of strategic plans is an issue though, since they are usually generated from the bottom up, without meaningful top-down fiscal constraints. Five-year plans therefore tend to be over-ambitious and many projects - either unfinished or never started - tend to cross over into the next planning period.

Prior to the PIL, the MPI and the MOF had little say over which project concepts would be developed. The PIL has improved things and now the two ministries, working together, must decide on the affordability, with the Prime Minister ultimately responsible for making the formal 'decision on investment intentions' of Group A projects. For projects of national importance, the MPI chairs the State Council responsible for independent appraisal of a projects, which advises the Government. For Group B and C projects, the MPI and MOF advises on affordability if the project is expected to use central government's budget, but decisions are taken internally by project promoters.

The MPI does not take a view on wider dimensions of projects, such as the project rationale/logic, potential social profitability, social and environmental sustainability, and risk, resulting in a narrow, but expanded, role under the PIL.

The decision on investment intentions locks in the costs of a project at an early stage and introduces a rigidity that negatively affects the performance of the rest of the PIM system.

3. Clarity of strategic objectives in terms of outputs and outcomes.

Strategic plans tend to be input and output focused and the upper hierarchy of the results chain (outputs $=>$ outcomes $=>$ impacts) tends to be underdeveloped or ignored.

Strategic infrastructure planning tends to be supply driven, with limited attention paid to demand for infrastructure services. This runs the risk of creating 'white elephant' projects.

4. Consideration of alternative approaches to achieving objectives.
Limited attention is paid to examining a range of strategic approaches to addressing identified problems or capacity constraints, and initial project solutions are not generally tested against alternatives. 


\section{Recommendations}

88. The preceding analysis of strategic guidance for public investment suggests the need to improve long term strategic planning of major infrastructure and reinforce the role of the MPI in first-level screening (investment intention stage) of large-scale projects, as well as mainstreaming the review by the MOF at this stage. At the same time, system design should support the reversibility, at appraisal, of a positive investment intention decision. More specifically, it is recommended that the authorities:

- Examine options for developing a rolling long-term infrastructure strategy for major projects (nationally important and Group A), straddling planning periods and helping to assure continuity.

- Review and amend the provisions of the PIL so as to give the MPI a stronger advisory role for aspects beyond affordability, including social profitability, demand, sustainability and risk, when appraising intention reports of budget-funded projects.

- Streamline and standardize MOF review of repayment capacity and ensure transparency and adequate timing of the review process, notably in relation to ODA projects.

- Introduce looser conditions for including ODA projects in the outer years of the MTIP, so that a signed loan/grant agreement is not necessarily a requirement if commitment in principle is in place.

- Review and revise the provisions of the PIL so as to make the investment intention decision a decision in principle only, to be confirmed and amended by subsequent decisions based on more in-depth analysis of project costs and benefits.

- Consider introducing a transparent prior decision step for large projects with significant fiscal impacts, before a pre-feasibility study is prepared.

- Prepare and disseminate methodological guidance on the preparation of a Pre-Feasibility Study and a Report on Investment Intentions, and develop training to facilitate implementation. 



\section{Project Appraisal}

\section{What Does Good Practice Look Like?}

89. Project appraisal is the PIM stage when the investment case for a project is analysed in depth and a decision made on the project's overall feasibility, and not just its technical feasibility. The preparation of a high quality feasibility study (preceded by a pre-feasibility study for larger and/or riskier project) is therefore central to project appraisal, at least for major projects ${ }^{34}$. sthe point of view of society as a whole, whether financial or otherwise, are positive. Conceptually, economic feasibility might be best conceived as'social profitability' ${ }^{\prime 35}$. For commercially-oriented projects, it is also important to determine financial feasibility, or the profitability of the investment from the point of view of the operating entity. For all projects, financial, environmental and social sustainability are also important elements of feasibility, as is the robustness of all dimensions of feasibility in the face of adverse risks. The realism of implementation plans and the effectiveness of project management arrangements should also be confirmed. The appraisal decision is a decision to proceed to detailed design ${ }^{36}$; it is not a decision to proceed to implementation, which will normally depend on confirmation of cost estimates at detailed design ${ }^{37}$ and on the availability of funding. The basis for the appraisal decision should also be subject to independent review, as discussed in the next section.

90. A strong appraisal process is a good 'investment'. Appraisal is the stage of the project cycle when flexibility over alternative approaches is highest and when amendment costs are lowest; so, from an efficiency perspective, it makes sense to focus planning and management effort at this stage, when changing course is less costly and more options are on the table. A weak appraisal process, allowing poor projects'through the gate', results in problems being discovered when adjustment costs are highest and flexibility lowest. The downside is that prior to detailed design and implementation, availability and reliability of information is low, and the risk of mistakes high. This indicates the potentially high pay-off from investing in a high quality feasibility study supported by statistically reliable data, before a funding decision is made and implementation commences. Figure 9 illustrates the above discussion in a graphic form, and is borrowed from the explanatory material for Norway's two-step quality at entry system, with QA1 corresponding to appraisal.

34 Lesser projects may be subject to a less detailed study, but there still needs to be some sort of assessment, even if it does not have the rigour of a full feasibility study.

35 Because there is some blurring of the difference between economic and financial feasibility in Vietnam, the term 'social profitability' will be used in this report to denote economic feasibility, as commonly understood in the international PIM literature.

36 Feasibility studies are usually based on a preliminary design and costings.

37 In good practice systems, the feasibility analysis is re-run with updated cost estimates to reconfirm the economic feasibility of the project. In the United Kingdom, for example, this analysis is known as the 'full business case'. 
Figure 9: A Strong Appraisal Process Reduces the Risk of Costly Project Adjustments during Implementation

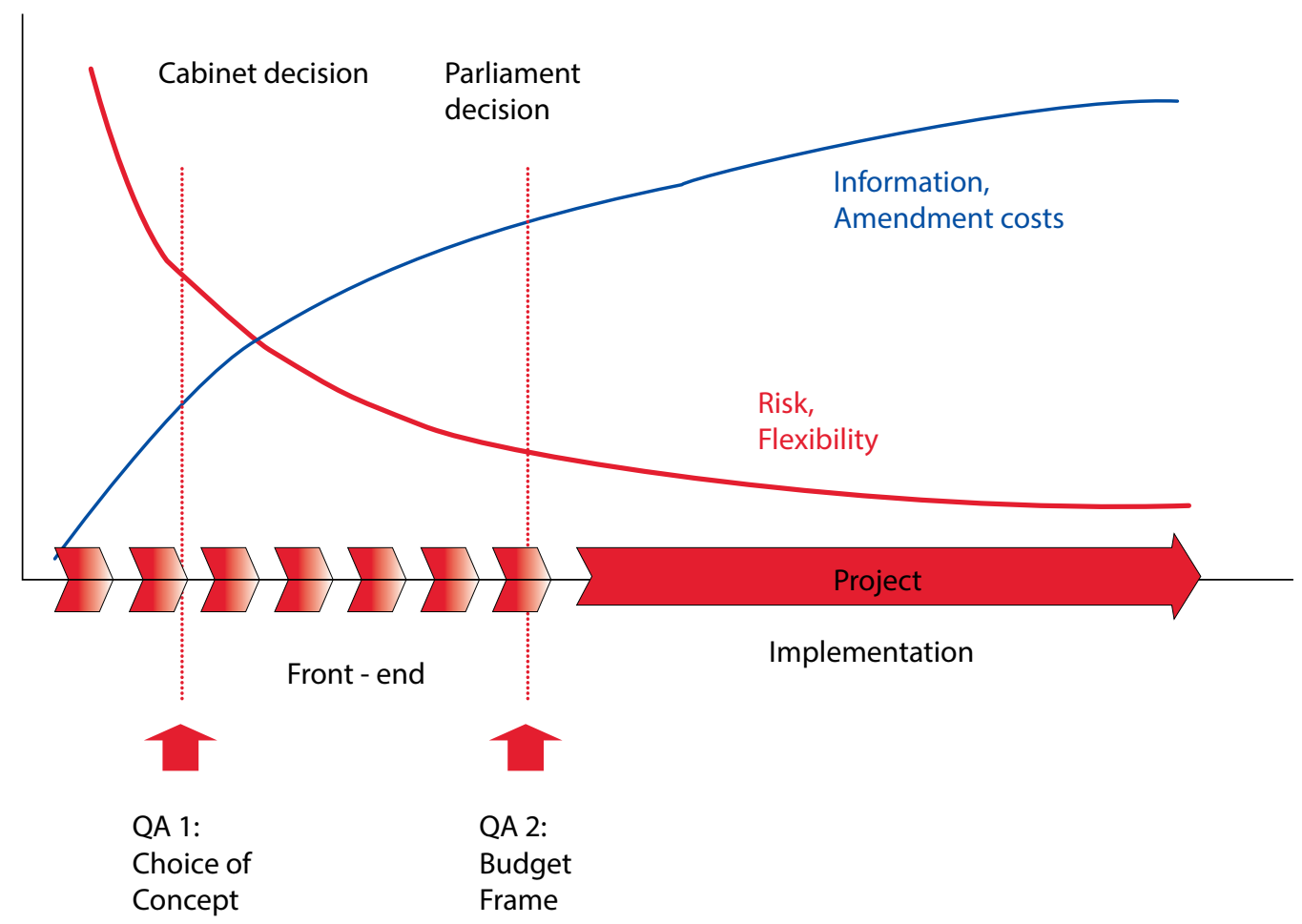

Source: Presentation on Quality-At-Entry by Ministry of Finance, Norway.

91. There are three main components of good practice in project appraisal:

- Publicised and transparent methodological guidance on project appraisal;

- Effective training and deployment of staff in project appraisal methods; and

- Disciplined, but proportionate, application of guidance on project appraisal, leading to a decision on economic and technical feasibility, and financial, environmental and social sustainability.

92. The default analytical approach at appraisal stage should involve the application of social cost-benefit analysis (SCBA - see Box 5), but there needs to be some flexibility. This is to allow: i) adoption of other methodological approaches - cost-effectiveness analysis and/or multi-criteria analysis - when it is not feasible to place monetary values on benefits and/or external costs; or ii) waiving of the requirement for SCBA for lower value or repeat projects where complex analysis may not be required or may not be an efficient use of scarce analytical skills. 


\section{Box 5: Social Cost-Benefit Analysis (SCBA)}

Social cost-benefit analysis (SCBA) aims to quantify in monetary terms as many of the costs and benefits of an investment proposal as feasible, including items for which the market does not provide a satisfactory measure of economic value. The purpose is to provide the information necessary to answer two key questions relating to efficiency and effectiveness:

- Does the project represent the best way of achieving the stated objective?

- Does the project represent the best use of the economic resources involved?

SCBA is a methodology for assessing the net benefits accruing to society as a whole (not just the operating entity) as a result of a project or program. It considers the flow of real resource costs and benefits over a project's life and includes estimating costs and benefits that are'un-priced' and not subject to normal market transactions, (e.g., public goods and negative and positive externalities). Allowances are made for distortions to market prices (through monopoly power, trade restrictions, labour market rigidities) and the value of indirect taxes and subsidies are excluded, as these represent transfers within society and not the real use of resources. Costs and benefits occurring at different points in time are quantified on a comparable basis by discounting to arrive at present values.

SCBA is usually a very important part of project preparation in advanced PIM systems and a key input into the appraisal stage. Its principle use is as a screening tool for identifying the set of good projects that can be then be considered for funding. It is also useful for prioritising projects within specific sub-sector investment programs, e.g., inter-urban roads, irrigation, power, and for choosing between different project design options. Because of its intensive use of skilled human resources, it is often better practice to subject only the largest projects (where mistakes are more costly) to economic analysis.

Where benefits are difficult to quantify, cost effectiveness analysis (CEA) of projects should be performed. This is analysis that compares the costs of alternative ways of producing the same or similar outputs and aims to determine the costs of achieving a specific physical target. Benefits are expressed in physical units and not money values; costs are expressed in money terms and discounted to determine present value. The results of the analysis are usually expressed as cost per unit output (or outcome if possible). CEA is useful in areas like health and education where it is more difficult (but not necessarily impossible) to quantify benefits in monetary terms.

Source: 'Strengthening Capital Budgeting in Georgia' Unpublished Policy Note for PER, World Bank 2010.

93. Even where a project is amenable to SCBA, the appraisal decision still needs to take into account important effects that are not captured. These will include consideration of benefits and costs that have not been valued, environmental impacts for example, and of differential impacts on disadvantaged groups, such as people in living in poverty or regions that are lagging economically. Financial and fiscal analyses are also important to determine a project's financial sustainability and the net impact on the public finances, both important factors to take into account when making the appraisal decision.

94. Whatever appraisal method (or combination of methods) is used, feasibility studies must include estimates of life-cycle costs of projects. It is not sufficient to provide estimates of capital costs alone, the appraisal decision must be made on the basis of comprehensive information on all costs over the planned life of a project, including operating and maintenance expenses. Maintenance expenses cover routine and periodic maintenance, including planned renovations. The estimates of operating and maintenance costs prepared for the feasibility study should then feed into forward budget estimates of projects eventually selected for funding (see Section 8). 


\section{Assessment of Project Appraisal in Vietnam}

\section{Recent legislative improvements have aligned Vietnam with many aspects of recognised good practice in project appraisal.}

95. Vietnam has adopted some important elements of recognised good practice in project appraisal and, through the PIL and the $\mathrm{CL}$, has established the preparation of a feasibility study as the basis for appraisal. The PIL and the CL have a clear definition of a feasibility study and require the preparation of such in order to inform an 'investment decision ${ }^{\prime 38}$, which clears a project to be submitted for funding. Prior to the PIL there was limited formal guidance for the preparation of a "project explanation report" (which is similar to a feasibility study) and actual practice was highly variable. The detailed scope of feasibility studies has been given legal basis through the PIL and the Construction $\mathrm{Law}^{39}$ and conforms in broad terms with international standards, including a requirement to assess financial and economic feasibility ${ }^{40}$, as well as other key dimensions of the project, like conformity with strategic plans, the need ('necessity') for the project, risks and environmental impacts. The factors to be taken into account in appraising a project and arriving at an investment decision are also established in the PIL and the Construction Law ${ }^{41}$, if only in general terms (see below).

96. As in good practice systems, there is a hierarchy of decision-making authority, which depends on project category ${ }^{42}$. This helps ensure that senior decision-makers focus on more important decisions. Investment decisions for projects of national importance, which have been approved by the National Assembly ${ }^{43}$, are taken by the Prime Minister. Heads of central bodies have authority to decide on Group A, B and C projects funded by central budget. The Chairman of the Provincial People's Committee decides on Group A, B and C projects funded from province financial resources, and the chairmen of districts and communes also have a role in deciding on projects at this level as well. The allocation of appraisal and decision-making rights is summarised in Table 6.

Table 6: Allocation of Responsibilities for Appraisal and Decision-Making in the PIL

\begin{tabular}{|lll|}
\hline \multicolumn{1}{c}{ Project Category } & \multicolumn{1}{c|}{$\begin{array}{c}\text { Pre-Feasibility Study/lnvestment } \\
\text { Intentions Report }\end{array}$} & \multicolumn{1}{c|}{ Feasibility Study } \\
\hline $\begin{array}{l}\text { Nationally Important Projects/ } \\
\text { National Targeted Programs }\end{array}$ & $\begin{array}{l}\text { A: State Appraisal Council } \\
\text { D: National Assembly }\end{array}$ & $\begin{array}{l}\text { A: State Appraisal Council } \\
\text { D: Prime Minister }\end{array}$ \\
\hline Category A & $\begin{array}{l}\text { A: Inter-ministrial Council or Appraisal } \\
\text { Leading Agency } \\
\text { D: Prime Minister }\end{array}$ & A: Technical Line Ministries \\
& A: Proposing LM/Province & Droposing LM/Provinces \\
\hline Category B & D: Proposing LM/Province & A: Technical LM/Province \\
\hline Category C & A: Proposing LM/Province & D: Proposing LM/Province \\
\hline
\end{tabular}

Source: Authors' Compilation from PIL; A: Appraisal; D: Decider; LM: Line ministry.

38 'Feasibility Study Report means an explanatory material for study contents about the significance, feasibility and efficiency of public investment programs and projects, which serves as a basis for the investment decision made by the competent authorities.' Article 4.3 of the PIL

39 Article 47.2 of the Public Investment Law and Article 54 of the Construction Law

40 'Analysis of investment efficiency including effectiveness and impacts on society, economy, security and defence; recovery of capital (if any)' at Article 47.2.n of the PIL and'... evaluation of socio-economic efficiency...' at Article 54.2.d. of the Construction Law.

41 Article 40 of the PIL and Article 58 of the Construction Law

42 Article 39 of the PIL.

43 Decision on investment intentions taken - see previous section. 
97. Investment decisions are made on the basis of an appraisal of feasibility findings undertaken by a body which is independent of the body which carried out the feasibility study. This is also in line with good practice. The PIL indicates the State Council for the Appraisal of Projects as being responsible for appraising projects of national importance. For other, non-construction projects, heads of central bodies and Chairmen of Provincial People's Committees should establish an appraisal council or appoint an investment management agency ${ }^{44}$. The Construction Law specifies clearly that 'Project-formulating organisations and individuals may not participate in the appraisal or verification of the projects they have formulated ${ }^{\prime 45}$.

Although Vietnam has adopted many elements of good practice in project appraisal, these have not yet been fully operationalised and the practical status of project appraisal in the broader system remains ambiguous.

98. Economic feasibility has moved to the centre of decision-making, but operationalisation is held back by an incomplete regulatory framework. The intention to make economic feasibility an essential part of the appraisal decision is evident in the new legal framework, but how this should be done in practice is not spelt out clearly enough to the various participants in the PIM system. Partly this results from some ambiguity in the primary legislation and supporting regulations. According to the PIL, the feasibility study should include 'analysis of investment efficiency, including effectiveness and impacts on society, economy, security and defence'. No further details on what this means in terms of indicators and methods is provided, even in the Law's guiding Decrees. Similarly, the Construction Law requires a feasibility studies to include an 'evaluation of the socio-economic efficiency of the project' with no reference as to appropriate evaluation methods or indicators.

99. Vietnam lacks formal guidelines on economic analysis methods for projects, as well as other aspects of feasibility studies. On top of the lack of precision in the legal framework (or perhaps because of it), there are no formal guidelines on rigorous methods for assessing economic feasibility (or 'social profitability'), such as SCBA (see Box 5). In the absence of centrally regulated methods, none of the national parameter values required for SCBA, such as a social discount rate or time values, are estimated, making consistent application of the tool difficult for those inclined to do so. The result is a patchy application of internationally recognised approaches to assessing economic feasibility, as reported in discussions with MPI and other stakeholders.

100. There is an incomplete understanding across government of the conceptual underpinnings of economic analysis ${ }^{46}$, in particular, of the differences with financial analysis (see Table 6 for a summary of the different perspectives). The result is that social welfare effects, which are not directly reflected in market transactions, are often dealt with purely in qualitative terms in the feasibility (and pre-feasibility) documentation. In this situation the perceived strategic importance or 'necessity' of the project often becomes the main driver of decision-making ${ }^{47}$, rather than the results of a quantified SCBA. It would, however, be misleading to say that quantitative approaches to valuing wider social effects are unknown in Vietnam, as discussed below.

44 Article 44 of the PIL.

45 Article 57 of the Construction Law.

46 To the extent that some regulations referring to 'economic analysis' in their titles are, in fact, referring to financial analysis on closer inspection.

47 Strategic relevance is important for project identification and prioritisation. Strategic relevance is usually seen as a necessary, but not sufficient condition for a positive appraisal decision: strategically relevant projects also need be demonstrated to be socially profitable. 
101. The evidence from the project samples gives support to the conclusion that there is uneven application of economic analysis in feasibility studies. Misunderstandings concerning the difference between economic and financial analysis are also confirmed. The responses from the survey of a sample of projects from the transport and education sectors are summarised in Annexes 1 and 2. The annexes indicate that feasibility studies were systematically prepared for the sample projects in both sectors. In transport, in five out of 10 cases, SCBA analysis clearly formed part of the feasibility study and in two cases it seems likely that this was the case ${ }^{48}$. Out of these seven potential cases, five were ODA-funded and two were bond-funded. The use of economic analysis therefore seems to be reasonably common practice in transport, but not universal. However, the sample does not include many Group B and C projects and only one project fully funded from the state budget; it is believed that the practice is far less common for these types of project. In education, the application of economic analysis was found to be less common, which is not entirely surprising given the difficulty of applying SCBA in this sector. Of the seven projects in the sample, only in two cases was economic analysis used and one of these cases is ambiguous. The unambiguous example was an ODA-funded university project. Overall, on the basis of the project sample, it can be concluded that feasibility studies involving economic analysis are performed in Vietnam, but not on a consistent basis and infrequently in social sectors (as in many countries). Fully developed feasibility studies are much more likely to be performed for ODA project and for major projects.

\begin{tabular}{|c|c|c|}
\hline \multicolumn{3}{|c|}{ Box 6: Main Differences between Financial and Economic Analysis } \\
\hline & $\begin{array}{l}\text { Financial Analysis - as applied } \\
\text { in financial CBA }\end{array}$ & $\begin{array}{l}\text { Economic Analysis - as applied in } \\
\text { social CBA }\end{array}$ \\
\hline Perspective & Agency/organisation/firm & Economy/society \\
\hline Objective & $\begin{array}{l}\text { Analysis of the net financial } \\
\text { impact of the proposal on the } \\
\text { agency }\end{array}$ & $\begin{array}{l}\text { Maximising the social returns to the } \\
\text { economy's resources }\end{array}$ \\
\hline Pricing & Market prices & $\begin{array}{l}\text { Shadow prices to correct for } \\
\text { distortions and/or opportunity } \\
\text { costs }\end{array}$ \\
\hline $\begin{array}{l}\text { Transfer payments (taxes \& } \\
\text { subsidies) }\end{array}$ & Included & Usually excluded \\
\hline Equity/distributional effects & Excluded & $\begin{array}{l}\text { Can be included, but usually treated } \\
\text { qualitatively }\end{array}$ \\
\hline $\begin{array}{l}\text { Externalities/Values of Public } \\
\text { Goods }\end{array}$ & Excluded & Included \\
\hline Depreciation & $\begin{array}{l}\text { Excluded (from discounted cash } \\
\text { flow analysis, but included in } \\
\text { financial statements. }\end{array}$ & Excluded \\
\hline
\end{tabular}

48 These two cases provide potential confusion between economic and financial analysis. It was stated that financial rather than economic analysis was performed, because neither project generates direct revenue streams it seems likely that economic analysis was performed and the wrong terminology used, financial analysis being interpreted as anything involving monetary values. 
102. In the absence of formal guidance on project appraisal methods, central agencies have no basis around which to package any technical support to project promoters. Neither the MPI nor the MOC currently provide direct technical support to project promoters during project preparation and appraisal, and there is no systematic capacity building effort. Lessons from other reforming countries demonstrate that bringing about an increased emphasis on social profitability and introducing new methodological approaches will require an initial capacity building effort of some significance, followed by continuous training and technical assistance thereafter. At present, the capacity to deliver this is absent and would need to be planned and created in time to support any agreed strengthening of the appraisal process.

103. While there is scope for improving the current appraisal process in Vietnam by adopting internationally recognised, expectations of what these approaches can bring should be kept realistic. The wider use of social cost-benefit analysis (SCBA) offers the opportunity for improved decision-making based on a more holistic assessment of the societal effects of public investment projects, both non-commercial and commercial. Although the wider application of economic analysis methods has undoubted advantages, it would, however, be mistaken to expect that such methods could be, or should be, applied to all projects, or that they can capture all dimensions of a project. If a decision is taken to apply SCBA more widely, the authorities will need to take account of the following factors:

- There are some non-market effects, particularly for social sector projects, where valuation is very difficult. In such cases, use of cost-effectiveness analysis (CEA), perhaps supported by a qualitative tool like multi-criteria analysis, will be more appropriate. The disadvantage of CEA compared to SCBA is that it does not provide an absolute measure of project worth, only a relative measure. Box 7 shows how one country, Chile, distinguishes between SCBA and CEA according to sector/sub-sector.

- Even where SCBA might be applied in theory, the research costs in valuing some effects suggest the need to apply this method sparingly and proportionately, confining its use to major, complex or innovative projects, as is the case in other countries. The principle of proportionality is already accepted practice in Vietnam where projects are categorized by importance for decision-making and could easily be extended to economic analysis.

- Finally, while the use of money values in SCBA has its advantages, it can also give a false sense of accuracy, and there is a danger of ignoring costs or benefits that are not easy or too expensive to quantify. 


\section{Box 7: Chile's Sector Guidelines on Analytical Methods}

Chile's general methodology for the presentation and appraisal of projects is supported by 32 sub-sector specific guidelines. As indicated in the table below, 18 of these guidelines concern the application of social cost-benefit analysis (SCBA) to projects in the sub-sector, 12 concern the application of cost-effectiveness analysis (CEA) and 2 offer the option of SCBA or CEA.

\begin{tabular}{|c|c|}
\hline Prescribed Methodological Approach & Type of Project Covered by Guideline \\
\hline Social cost-benefit analysis & $\begin{array}{l}\text { 1. Airports } \\
\text { 2. Fishing ports } \\
\text { 3. Low standard roads } \\
\text { 4. Bicycle paths } \\
\text { 5. Fluvial defences } \\
\text { 6. Public buildings } \\
\text { 7. Rural electrification } \\
\text { 8. Multipurpose dams } \\
\text { 9. Traffic management } \\
\text { 10. Ancillary investments related to roads } \\
\text { 11. Small airfields } \\
\text { 12. Equipment replacement } \\
\text { 13. Burying electric cables } \\
\text { 14. National network of community telecenters } \\
\text { 15. Rural telephony } \\
\text { 16. Interurban transport } \\
\text { 17. Intermediate roads } \\
\text { 18. Urban roads }\end{array}$ \\
\hline Cost-effectiveness analysis & $\begin{array}{l}\text { 1. Drinking water } \\
\text { 2. Replacement street lighting } \\
\text { 3. Primary healthcare } \\
\text { 4. Sports facilities } \\
\text { 5. Educational projects } \\
\text { 6. Juvenile housing and detention centers } \\
\text { 7. Police infrastructure and equipment } \\
\text { 8. Regional development master plans } \\
\text { 9. Jails } \\
\text { 10. Sustainable reconstruction plans } \\
\text { 11. Information technology } \\
\text { 12. Police surveillance }\end{array}$ \\
\hline SCBA or CEA & $\begin{array}{l}\text { 1. Rainwater drainage } \\
\text { 2. Residential waste management }\end{array}$ \\
\hline
\end{tabular}

Source: 'Institutional Safeguards for Cost-Benefit Analysis: Lessons from the Chilean National Investment System', Andres Gomez-Lobo, Journal of Benefit-Cost Analysis, Volume 3, Issue 1, 2012.

104. Based on international experience, various options are available for establishing the methodological basis for SCBA. Some countries, like the UK and Ireland, have an overarching national methodology and allow sector ministries to develop their own methodologies, provided these remain consistent with the national guidance. At the other extreme, Chile has an elaborate system of sectorspecific methodologies developed at the centre of government (see Box 7). In between these two cases, the Netherlands and the European Commission ${ }^{49}$ amalgamate the global and sector guidance,

49 'Guide to Cost-Benefit Analysis of Investment Projects', Directorate General for Regional and Urban Policy, European Commission, 2014. Gives general guidance on principles followed by specific guidance on: transport, environment (including water); energy; broadband; and research \& development. 
although the latter is much less detailed than can be provided in individual guidelines. As an example, Box 8 gives the table of contents of the Netherlands' methodological guidance for SCBA.

105. The legal status of methodological guidelines varies between countries. Ireland's guidelines are advisory, while the UK's are 'binding' with their force coming from the powerful position of the finance ministry within government. In Chile, the use of guidelines is enforced through legal means, which is most likely to be the case in Vietnam. Whether guidelines apply to all projects is another issue. In Ireland, only projects with a value in excess of euro 20 million are subject to SCBA and other projects use simpler methods; in the UK and the Netherlands, methods apply to all projects, but the level of effort (financial and human) in applying them is expected to be proportionate to project size.

\section{Box 8: The Netherlands' General Guidance for Social Cost-Benefit Analysis}

The main contents of the General Guidance for Social Cost-Benefit Analysis are:

1. Introduction

2. Role of social cost-benefit analysis (SCBA) in decision-making (and its limitations)

3. Principles of social cost-benefit analysis, including the concept of consumer surplus (and its measurement), market failure and public goods

4. Research steps for preparing SCBA -8 steps in the preparation of a SCBA

5. Preparation of a SCBA - including identification of the base case and project alternatives

6. Impact assessment - including methods for determining effects

7. Determining the benefits - including valuation principles for goods/services for which there is no market and discounting

8. [Short] Sector-specific guidance:

- Transport and mobility

- Spatial development

- Care and health

- Flood protection

- Energy and environment

- Natural environment

- Education

- Labour market

9. Cost estimates - including differences between market and social values, and valuation principles

10. Uncertainty and risk - including risk analysis and valuation

11. Reporting, presenting and interpreting the results of SCBA

Source: General Guidance for Cost-Benefit Analysis, Gerbert Romijn and Gusta Renes, Netherlands Bureau for Economic Policy Analysis and Netherlands Environmental Assessment Agency, 2013

106. Proportional application of appraisal guidelines is to be recommended, but international experience indicates different approaches to proportionality. Appraisal is intensive in its use of financial resources and skilled human resources, and the effort dedicated to it should be proportionate to the scale of the project. There are different ways of dealing with this: either the same methods will be recommended for all projects, but the effort reduced for smaller projects, as is the case in the 
Netherlands $\mathrm{s}^{50}$ and United Kingdom ${ }^{51}$; or more sophisticated methods will only be required for major projects, as is the case in Ireland as shown in Table 7. In practice, the latter solution may be preferable in low capacity environments, although careful consideration of the threshold for applying SCBA will be required. Ireland's threshold is high by international practices ${ }^{52}$ and the requirement for SCBA usually kicks in at a lower project cost. The threshold will be very country specific, and the decision on where to place it should start from an analysis of the size distribution of projects by sector, together with an assessment of available analytical capacities.

\section{Table 7: Proportionality in the Application of Assessment Tools in Ireland}

\begin{tabular}{|c|c|}
\hline Project Value & (Minimum) Type of Assessment \\
\hline$<€ 0.5$ million & $\begin{array}{l}\text { Simple assessment for minor projects, such as those involving minor } \\
\text { refurbishment works, fit-outs, etc. }\end{array}$ \\
\hline$>€ 0.5$ million $<€ 5.0$ million & $\begin{array}{l}\text { Single appraisal (combining elements of Preliminary Appraisal and } \\
\text { Detailed Appraisal) }\end{array}$ \\
\hline$>€ 5.0$ million $<€ 20.0$ million & Multi-criteria analysis of options \\
\hline$>€ 20.0$ million & $\begin{array}{l}\text { Cost-benefit analysis or cost-effectiveness analysis (depending on the } \\
\text { extent to which monetary values can be estimated for benefits) }\end{array}$ \\
\hline
\end{tabular}

107. There is scope for Vietnam to develop more structured appraisal criteria for assessing feasibility studies. Appraisal criteria are defined in general terms in the legal instruments. This is appropriate in primary legislation, although the criteria in the PIL are probably still too general ${ }^{53}$. In regulations and guidance, more structured criteria are required to guide those preparing feasibility studies and to ensure structured, consistent and transparent appraisal of feasibility studies by those responsible for this task. The checklist used by the UK finance ministry provides one such example ${ }^{54}$.

108. Compared to the earlier investment intentions decision, the investment decision and the feasibility study, upon which it is based, do not have a high standing in the Vietnamese PIM system, as implemented. While the PIL and Construction Law appear to place the investment decision in high importance, the reality is that it is not a decisive step. The evidence for this is that, as much as can be ascertained ${ }^{55}$, few, if any, projects drop out at this stage. If appraisal were working as anticipated in the good practice model, a sizeable number of projects should be expected to proceed no further. In Vietnam, the focus of the feasibility study and the appraisal that follows is largely on confirming the technical design and cost estimates, and limited attention is paid to typical feasibility criteria, like social profitability, sustainability factors and risk, in arriving at a decision. The relative unimportance of the analysis performed for the feasibility study - except for design and costing compared to the pre-feasibility analysis is conformed in discussions with the MPI and from the MOT's general submission as part of the project sample review.

50 'The effort and resources required for the preparation of a SCBA should be proportionate to the importance of the measure.' Kabinetsbrief bij de algemene MKBA Leidraad, Minister of Finance, December 2013.

51 'The effort departments [ministries] expend on developing the proposal should be proportionate to the likely costs and benefits', Assessing Business Case: a Short Plain-English Guide, HM Treasury

52 It was higher, but has been lowered as capacities have developed.

53 '1. Socio-economic strategy and plan; 2. Socio-economic and sectoral development planning; 3. Significance of public investment program and project; 4. Objective of public investment program or project; 5 . Investment intention decided by competent authorities; 6 . Capability of mobilizing and balancing investment portfolio and other funds for investment program or project.' Article 40 of the PIL.

54 Checklist of Assessment of Business Cases, HM Treasury

55 Information on the drop-out rate is not collected, but discussants were unable to identify cases of projects being turned down at this stage on the basis of feasibility findings. 
109. Although the cost estimate in the feasibility study should be more reliable ${ }^{56}$, it is the estimate prepared for the $\mathrm{PFS} / \mathrm{RO} \mathrm{II}^{57}$ and already approved through the investment intentions decision that forms the basis for the investment decision. The estimate in the investment intentions decision is important because it is used in formulating the MTIP and represents the opinion of the MPI/MOF on the affordability of the project. This downgrades the importance of the feasibility estimate, which cannot come out higher (and thus put into question the project's affordability). If the FS indicates that costs will be higher, then projects must be re-scoped - activities and outputs reduced or quality standards sacrificed - to stay within the originally agreed figure in the investment intentions decision. If FS estimates come in lower, opportunities for 'gold-plating ${ }^{\prime 58}$ arise and even the possibility of idle budget allocations. As will also be discussed in the next section (Independent Review), the absence of the MOF/MPI from the appraisal stage introduces a rigidity that can lead to projects being less effective than they might be ${ }^{59}$ if the affordability of upward adjustments to estimated costs could be re-examined at this stage. This also introduces the possibility of inflated estimates being proposed in the PFS/ROIl to get round early restrictions on total costs.

There are some features of the design of Vietnam's new project appraisal process that are not compatible with the good practice model.

110. The different treatment of non-construction and construction projects at the project appraisal stage is not helpful to the integrity of the PIM system. Similar to first-level screening (the previous PIM stage), non-construction and construction projects are subject to a different legal framework when it comes to project appraisal. The PIL establishes feasibility requirements and appraisal responsibilities for non-construction projects and ODA-funded projects, but defers to the Construction Law for construction projects, which establishes separate feasibility study and appraisal requirements for these projects. The feasibility study contents in the Construction Law, while being more detailed, are broadly similar to those in the PIL, but not completely consistent, leading to different possible interpretations of requirements. When it comes to appraisal, there is some inconsistency between the two laws. With the exception of the State Appraisal Council for projects of national importance, the Construction Law makes no mention of the formation of appraisal councils ${ }^{60}$, as authorised by the PIL, allocating responsibility instead to specialised construction agencies (which is also foreseen in the PIL, just not exclusively). The two laws are, however, consistent when it comes to decision making, with the Construction Law deferring to the PIL for most forms of funding ${ }^{61}$. This divergence between the treatment of construction and non-construction projects does not exist in countries with effective PIM systems and is a potential hindrance to a consistent project appraisal process.

111. Appraisal and decision-making responsibilities differ depending on funding. Except for variations relating to project size/importance, it is usual practice for PIM systems to be designed so that projects are appraised and approved according to the same procedure to ensure consistency, irrespective of the source of the public financial resources involved. In Vietnam's system, there are variations according to the source/nature of funds. For example, different decision-making process for

56 According to the Construction Act, the feasibility estimate is prepared on the basis of a 'basic design' incorporating regulated design standards and cost norms.

57 The PFS estimate is based on a preliminary design and will be built up from the unit costs of the specified outputs.

58 'Gold-plating' means unnecessary over-specification of designs.

59 A project where the outputs or the quality of outputs are reduced will be less successful in delivering outcome and impact, to use the terminology of the results chain.

60 Article 57 of the Construction Law.

61 '...the competence to decide on construction investment must comply with the law on public investment.' Article 60.1 of the Construction Law. 
projects using government guarantees are foreseen in the Construction Law ${ }^{62}$ and the PIL foresees different procedures for ODA projects.

112. In neither the PIL nor the Construction Law is there any requirement to examine project alternatives, either at PFS of FS. A critical feature of a feasibility study (and a pre-feasibility study) is that it should compare the proposed project to alternatives and ascertain whether it represents the best solution - in both technical and economic terms - to the identified problem or constraint. Alternatives range from completely different solutions (including policies not requiring investment) to technical variants on and alternative phasing of the preferred project solution. The former are more likely to be examined at PFS stage and the latter at FS stage. This concept of this purpose of a feasibility study is missing from the legislative framework and ignores one of the fundamental purposes of economic appraisal (see Box 4).

113. The legislative framework also makes no explicit mention of any requirement to determine the demand for the services of the proposed investment. While a demand assessment might be taken to be implicit in the requirement to demonstrate the 'necessity for the investment', this phrase could also be interpreted in other ways. The absence of an explicit reference to demand forecasting, together with frequently repeated assertions among technical ministries that infrastructure supply creates its own demand, is a cause for concern. Accurate demand forecasts are an essential input into valuing benefits as part of a SCBA but, even for projects where SCBA is not feasible, a minimum requirement should be to demonstrate that there is demand for the additional capacity created by the project. Failure to consider, and accurately quantify the adequacy of demand is one of the reasons for the many 'white elephant' projects across the world, in advanced as well as less advanced economies, especially in the social, cultural and sports sectors, but also in the transport sector. Box 9 gives some examples of poor demand forecasting for transport infrastructure projects in Europe.

62 Article 60.2 of the Construction Law 


\section{Box 9: Examples of Demand Shortfalls for European Transport Infrastructure Projects}

\section{European Union Funded Airport Infrastructure}

'The EU auditors examined investment projects at 20 airports - in Estonia, Greece, Italy, Poland and Spain - which received more than $€ 600$ million of EU money from 2000 to 2013. The auditors found that only half of these airports could show the need for EU-funded investment and that funded infrastructure was often underused, with some $€ 38$ million worth not being used at all.

Only half of the airports audited increased passenger numbers, while, for more than half of them, air traffic forecasts significantly over-estimated increases. In Cordoba, for example, fewer than 7,000 passengers travelled in 2013, against the 179000 forecast. In addition, for most airports there was little evidence of an improvement in customer service or of regional socio-economic benefits, such as the creation of additional jobs.

Seven of the airports, mostly those with fewer than 100,000 passengers peryear, are not financially self-sustainable and will struggle to remain in operation without more public money. In Greece, for example, Kastoria's revenue of $€ 176,000$ for 2005- 2012 was dwarfed by the $€ 7.7$ million it cost to keep the airport open over the same period. The auditors concluded that a further $€ 16.5$ million invested in an extension to Kastoria's runway (which has never been used for the type of aircraft for which it was built) cannot be considered an effective use of public funds.'

Source: 'EU-funded investments in airports provide poor value for money, say EU auditors', Press Release following publication of performance audit report, European Court of Auditors, December 2014

\section{Other Transport Infrastructure}

'For the Channel tunnel, more than five years after opening to the public, Eurostar train passengers numbered only 45 percent of that forecasted for the opening year; rail freight traffic was 40 percent of that forecasted; the result has been several near-bankruptcies. For the Humber bridge in the UK, 16 years after opening to the public actual traffic was still only about half of that forecasted. In Denmark, it took more than 20 years for actual traffic on the New Little Belt bridge to catch up with forecasted traffic, and for several years the difference between forecasted and actual traffic grew larger instead of smaller.'

Source: 'Inaccuracy in Traffic Forecasts', Bent Flyvbjerg, Mette K. Skamris Holm, and Søren L. Buhl, Aalborg University, Denmark

\section{Vietnam has not yet brought climate change into the mainstream when it comes to PIM.}

\section{Climate change resilience does not feature strongly in current appraisal practice in Vietnam.}

In 2013, the MPI had already issued Decision 1485 (October 17, 2013) which introduced a Climate Change Adaptation Prioritization Framework (APRF) to guide socio-economic development planning. The APRF is a decision support tool designed to enable the Government to prioritize and mainstream climate change adaptation actions/projects in the SEDP. The MPI has also issued a detailed guidelines for the application of APRF in planning practice. While the transport projects in the sample are not climate change adaptation projects, several of them are located in areas which are highly vulnerable to climate changes. Yet only one project out of three transport projects approved since Decision 1485 included climate change resilience in the feasibility study and in no cases was it considered during appraisal. Responses for half the projects did, however, indicate that an environmental impact assessment in line with international good practice had been performed. The findings from the project sample on climate change resilience are summarised in Table 8. 


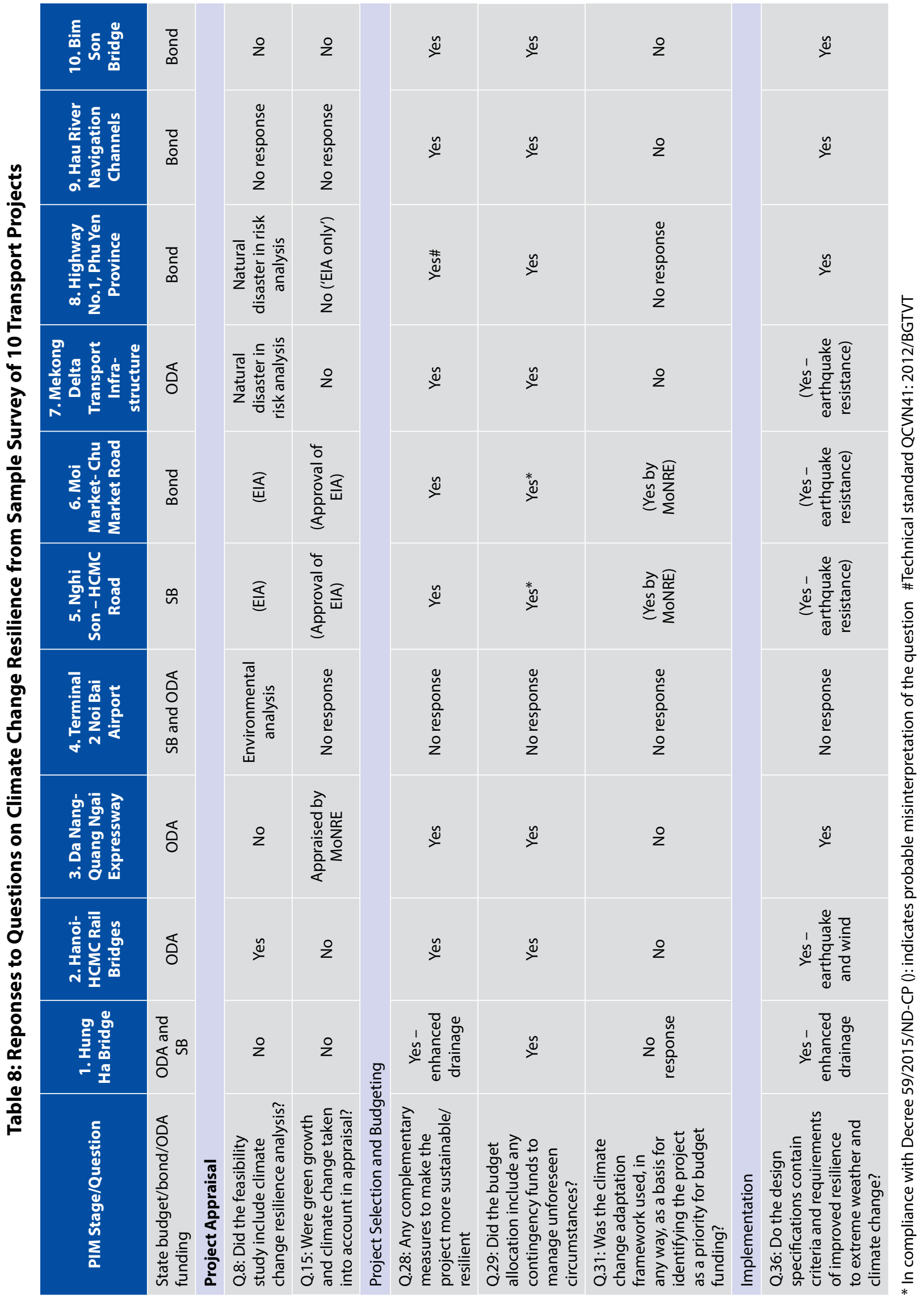




\section{Summary Assessment of Formal Project Appraisal}

\section{Key Functionality}

Projects that pass first-level screening undergo more rigorous scrutiny of their social profitability (economic feasibility) or costeffectiveness, among other things.

\section{Desirable Regulatory,}

Administrative and

Technical Arrangements

1. Publicised and transparent methodological guidance

2. Effective training and deployment of staff in project appraisal methods

\section{Disciplined, but proportionate, application of guidance on project appraisal}

\section{Current Arrangements in Vietnam}

By requiring an appraisal decision based on a feasibility study, which includes some assessment of economic efficiency, Vietnam has adopted an important feature of PIM good practice. Unfortunately, the scope and content of the feasibility study is not regulated in sufficient detail and there is no official guidance on the methodologies that should be applied, especially social cost-benefit analysis and related cost-effectiveness analysis. In the absence of formal guidance, there are also no official estimates of key parameters required for economic analysis, such as the social discount rate.

Central agencies offer little technical support to investors in the detailed application of the Public Investment Law.

There is no centrally organised and systematic training of officials on project appraisal methods incorporating economic analysis (as differentiated from financial analysis) and its conceptual underpinnings and usefulness are not widely known. In the absence formal methodological guidance, there is no consensus on what training might involve and how to organise it.

The absence of formal guidance makes it difficult even for willing investors to examine the economic feasibility (or 'social profitability') of project proposals in a consistent way, and the general practice is to examine social profitability in qualitative terms only. Where a quantified estimate of social profitability is infeasible - some social sectors for example quantitative analysis of demand and cost-effectiveness analysis, the minimum requirements, are generally not performed.

There is apparent discipline in the new appraisal and decision-making process, but in practice the investment decision - resulting from appraisal of the feasibility study - is not as important as the prior investment intentions decision, when project costs are fixed. Very few projects proposals are stopped at appraisal.

Construction and non-construction projects are regulated differently and subject to different appraisal procedures. This dichotomy is difficult to understand and is not consistent with international PIM practices, which entail a unified system. 


\section{Recommendations}

115. The preceding analysis of system weaknesses in appraisal suggests the need to raise the status of the investment decision so that it becomes the critical decision of the 'quality-at-entry' process. This will also require creating the necessary regulatory, technical and capacity conditions for making social cost-benefit analysis the default analytical tool for appraisal decisions. Specifically, it is recommended that the authorities take steps to:

- Raise the status of appraisal and the resulting investment decision so that together they represent a critical checkpoint in the PIM system, through which wasteful and ineffective projects are prevented from passing. The rejection rate is an indicator of system performance and should be monitored.

- Introduce greater clarity and consistency into the regulation of the contents and applicable methods for feasibility studies so as to bring Vietnamese practice into line with international standards, especially with respect to the analysis of economic feasibility/social profitability of projects and attention to the life-cycle costs of projects.

- Develop methodological guidance on analytical tools for use in feasibility studies, including social cost-benefit analysis, and regulate for the consistent application of the guidance. These methods should apply both to public projects with a commercial orientation, e.g., utilities and certain transport services, and those without, e.g., social facilities and non-tolled roads, with adaptations for projects where valuation of benefits is not feasible. The guidance should be expected to draw from similar guidance prepared by other countries or international bodies.

- Tailor the new methodological requirements to the scale and complexity of projects, and to the capacities of those required to implement them. This may mean limiting the use of social cost-benefit analysis to the largest and most complex projects, at least to begin with.

- Operationalize the technical guidance by designing and implementing a program of research for estimating and disseminating national parameter values for use in social cost-benefit analysis and cost-effectiveness analysis. At a minimum, this requires estimation and approval of a social discount rate for Vietnam. Other values that it is useful to determine centrally are the value of time and the value of a statistical life.

- Develop, regulate for and apply more specific appraisal criteria for use in appraising projects on the basis of feasibility studies.

- Review the parallel systems for non-construction projects and construction projects and design a unified appraisal process.

- MPI to undertake an assessment of capacity building needs across government and develop a phased training program to support gradual roll-out of an improved process. Accompany the training with a centrally managed on-the-job technical assistance program. 


\section{Independent Review of Project Appraisal}

\section{What Does Good Practice Look Like?}

116. Independent review of appraisal findings is required to incentivise quality, to challenge politically driven projects and to counteract planners' optimism. The motivations for independent review are that:

- The incentives for a sponsoring entity to deliver a high quality project proposal may be weak, even though it may be in possession of the best information to formulate and analyse such a proposal. An independent reviewer can provide external pressure for raising quality standards.

- Officials in an agency sponsoring a project may find it difficult to challenge 'momentum' projects that have been championed by the political leadership of that agency. An external reviewer is not subject to the same pressures and can better contest decisions which are not supported by strong analysis.

- There is a demonstrated tendency across countries and through time for project planners to over-estimate the benefits and under-estimate the costs of their proposals. This is known as 'optimism bias' (See Box 10), and impartial review is one of the few ways to identify and correct for it.

\section{Box 10: The Problems of Optimism Bias in Projects}

Optimism bias is the systematic tendency for project costs to be under-estimated and for project benefits to be over-estimated. A research group on large infrastructure at Aalborg University (Denmark) tried to quantify the extent of the problem and offer explanations, working from a large sample of major transport projects. This research (summarized in Flyvbjerg, 2005) finds that significant cost over-runs were seen across a sample of 258 major transportation projects, irrespective of country, continent or transport mode, and with no tendency to diminish. Nine out of 10 projects had a cost over-run, and the average (real) cost overrun was 45 percent for rail, 34 percent for bridges and tunnels and 20 percent for roads. From a sample of 208 rail and road projects, nine out of 10 rail projects had overestimated traffic, with actual passenger traffic 51 percent lower on average than forecast.

Further research on large dams found that three out of every four large dams suffered a cost overrun in constant local currency terms and that actual costs were on average $96 \%$ higher than estimated costs. The research also found that systematic cost overruns were experienced in every region of the world.

Because errors are systematically biased in one direction, poor techniques cannot explain the bias. The plausible explanation is that project promoters and planners systematically make projects look better than they are to obtain funding approval.

Sources:

'Policy and Planning for Large Infrastructure Projects: Problems, Causes and Cures', Bent Flyvbjerg, World Bank Policy Research Working Paper, no. 3781, 2005.

'Should We Build More Large Dams? The Actual Costs of Hydropower Megaproject Development', A. Ansar, B. Flyvbjerg, A. Budzier, and D. Lunn, Energy Policy, 2014 
117. It is good practice to subject appraisal findings and recommendations, together with the underlying studies, to review by an independent party. The underlying assumptions, forecasts and conclusions put forward by a project sponsoring entity need to be challenged to test their robustness and realism. Box 11 is a summary of the kinds of question that are asked of projects during independent review in France. Ideally, independent review should be proportionate, so the depth of the review for small or repeat projects may be lower.

\section{Box 11: Questions for Independent Reviewers in France}

The independent review ('second opinion') in France should respond to the following questions:

- Does the project documentation respond to the documentary requirements of a socio-economic evaluation?

- Have the methodological guidelines and ministerial instructions, where these exist, been properly followed. Have the prescribed parameter values for appraisal been respected?

- How have the non-monetized effects of the project been taken into account where these are critical for the evaluation of the project?

- Is the scope of the evaluation appropriate to the project?

- What method has been used and how does this compare with recognized practices in other comparable sectors and in other countries?

- Are the input parameters realistic and coherent?

Source: 'Évaluation des grands projets d'investissements publics' Annexe au Projet de Loi de Finances 2015.

\section{There are four main components of good practice at independent review stage, as follows:}

- Independent checks to ensure objectivity and quality of appraisals.

- Disciplined completion of projects appraisals prior to budget preparation, so that independent review is not rushed and squeezed by the budget preparation calendar.

- Identifying and maintaining an inventory of appraised projects ranked by priority for budgetary consideration.

- Clarity of roles for independent review, between projects that are minor and may be dealt with at the departmental level and those that require additional scrutiny.

\section{Institutional Arrangements for Independent Review:}

119. Meaning of 'independence': Independence does not mean external to the government. The independent reviewer function should be performed by an objective body with nothing to gain from the project going ahead. Ideally, this body should be external to the proposer or appraiser, but still within the public sector. The public sector body charged with independent review may make use of private sector consultants (as is the case for major project in Norway) or experts from elsewhere within the public sector (as is the case in the UK, where project reviewers are trained and certified).

120. Coverage of independent review: Appraisals for projects should be subject to some form of objective review, even if this is done by another part of the organization appraising the project. It may not be practical for the objective review to be carried out by the nominated external organization for independent review, which may need to specialize in major and riskier projects because of capacity 
constraints ${ }^{63}$. If this is the case, the appraisal for lower value projects should at least be reviewed by a part of the proposing/appraising organization that has no direct interest in the project going ahead.

121. Depth of review: Independent review varies from country to country in the depth of the analysis performed as illustrated in Box 12.

\section{Box 12: Country Differences in the Depth of Independent Review}

- In the United Kingdom, where the economics and finance ministry only reviews major projects, a checklist approach is used to ensure that the fundamentals of the appraisal methodology have been followed and to question critically the appraisal findings and underlying assumptions. For all projects, the Gateway Review System, which is internal to the agency sponsoring the project, provides for review by external peer reviewers from elsewhere in the public sector.

- In Chile, all projects are subject to review by the planning ministry but, because of the scale of the task, this is essentially a quality control process - and less of a 'second opinion' - to ensure that the highly detailed methodologies have been applied properly .

- In France, the General Commission for Investment organizes a second opinion on appraisals of major projects ( $€ € 20$ million), which critically examines both the methodological approach (to ensure conformity with guidelines), the calculation of parameters used in the appraisal and the appraisal findings.

- Unusually, in South Korea, independent review precedes appraisal and involves the preparation of a preliminary feasibility study for major projects (>US\$ 50 million) as a basis for making a decision to proceed to appraisal. This involves much deeper analysis by the independent reviewer, PIMAC, than in other countries, but, combined with a system for controlling cost escalation, it has proved remarkably effective.

Source: Guidelines on Public Investment Management, World Bank, forthcoming.

122. Outcome of independent review: It should be difficult for a project to proceed further if it has been the subject of a negative independent review. This being said, completion of an independent review is not necessarily a decision point and the independent reviewer will often only advise the final decision-maker. Country variations in the authority attaching to independent review findings are illustrated in Box13.

63 In the UK for example, the finance ministry only reviews and approves appraisals of projects that are classified as major projects included in the Government Major Project Portfolio. Major projects are defined in terms of value, risk and sectoral characteristics. 


\section{Box 13: Country Variations in Outcome of Independent Review}

- Chile's system is recognized as being rather strict, but even here some projects proceed each year without positive reviews from the planning ministry.

- In France, the General Commission for Investment prepares an opinion for the Prime Minister, which goes also to the minister proposing the project and to parliament.

- In Ireland, the review by the Central Expenditure Evaluation Unit is purely advisory for the minister proposing the project.

- In the Netherlands, it is specified that the findings of independent review should be taken into account in the final decision, but there is no requirement for the decision to be determined by the review findings.

- In the United Kingdom, independent review is closely tied to a decision point ('Treasury Approval Point') and projects will not proceed further if negatively reviewed.

- In South Korea, it is legislated in the Public Finance Law that the independent review by PIMAC is mandatory in major projects (>US\$ 50 million) for the decision making of the Ministry of Strategy and Finance, and the decision is closely tied to the result of the independent review in practice.

Source: Guidelines on Public Investment Management, World Bank, forthcoming.

123. Timing of independent review: in recognition of the fact that appraisal and budgeting are separate PIM processes and to allow sufficient time for independent review, it is usually not linked directly to the budget preparation calendar, which only provides deadlines by which review must be completed for a project to be considered for funding in a particular budget year. The time usually taken for independent review varies considerably between countries and reflects the depth of the analysis performed (see above). In Chile, the planning ministry responds in 10 days once the project has been assessed as admissible (for which decision 5 days are allowed). In the United Kingdom, the turnaround time for a decision at a Treasury ${ }^{64}$ Approval Point is 28 days. France allows 1-4 months for a second opinion to be prepared once the project documentation is received. South Korea's much more elaborate preliminary feasibility study takes around 4 months and may be extended if the work on the preliminary feasibility study indicates some alterations to the project concept.

\section{Assessment of Independent Review in Vietnam}

The necessity for some form of independent review of project proposals is recognized in the legislation, but the design falls short of a fully impartial review of the feasibility study and appraisal findings.

124. The concept of independent review of project proposals has been established in the new legal framework, but the review associated with earlier first-level screening takes on greater prominence and has more objectivity than a later review at appraisal stage. Section 5 on first-level screening discusses the review process which informs the investment intentions decision. It identifies the narrow focus on affordability of the review by the MPI/MOF, and the fragmented responsibilities, with the MOC having responsibility to review the cost side of PFS/ROIl submissions. A review of criteria like strategic relevance, the project rationale, and the demand for project services is outside the formal mandate of the MPI or MOC at this stage, although these factors are covered (but to limited effect).

64 In the UK, the 'Treasury' is the finance ministry. 
125. Appraisal includes some arrangements which have the semblance of an independent review function, but on closer examination lack the necessary degree of objectivity. The important stipulation in the Construction Law 2014 that those appraising projects should be different from those formulating them has been mentioned already. As described in the previous section, for projects of national importance a State Council for Appraisal is constituted to appraise a project (on the basis of the feasibility study) and advise the Prime Minister on the investment decision. Similarly, for other non-construction projects, heads of central agencies who are responsible for the investment decision must be advised by a specially constituted appraisal council or by a specialized investment management agency, which are responsible for appraisal of the feasibility study. For construction projects, heads of central agencies should also turn to the investment management agencies for an independent appraisal to inform the investment decision.

\section{Vietnam's appraisal procedures have been designed to introduce an element of objectivity} into the decision-making process, but fall short of including a properly specified independent review function. Prior to the PIL and the Construction Law 2014, the concept of objective appraisal was almost non-existent, so the improvements should be recognized. Even so, under the new arrangements, the level of detachment of the various parties from the appraisal decision may be questioned, suggesting the need for a further level of review by a party with no interest at all in the outcome of the appraisal and the investment decision. For example, the State Council for Appraisal is made up of ministry representatives, including the ministry proposing a project, and is likely to be, de facto, more of a bargaining arrangement than a disinterested arbiter ${ }^{65}$, in spite of the MPI's role as chair. Similarly, it is difficult to ensure that the membership of appraisal councils will be sufficiently impartial. The alternative of using an investment management agency results in implicit pressures from the client-contractor relationship, because the agency will have been employed by and will be affiliated $^{66}$ to the project owner.

\section{Both the MPI and the MOF are presently excluded from any sort of independent review} function at the appraisal stage. At central government level, technical line ministries, like MARD, MOT and MOIT are responsible for appraisal of projects directly in their areas of responsibility, i.e., agriculture, irrigation, transport and heavy industry. At the same time, MOC exercises a cross-cutting, independent review function for all construction projects, which involves it in reviewing, for example, construction projects under MOET, and even light industrial projects ${ }^{67}$. The MOC's preoccupations, however, lie largely in the technical design of construction projects and whether costs have been estimated according to regulated norms; it takes little interest in the broader question of whether a convincing and robust case has been made for committing public financial resources. This means that neither the MPI nor the MOF have a chance to revisit their opinions on projects should there be major changes between the PFS and the FS. The preceding analysis has shown that such changes can occur. While less frequent, significant changes to capital costs may occur; more likely, there will be (usually downward) revisions to activities/outputs that could diminish forecast benefits. Both cases, call into question the economic viability of the project and would suggest a need for re-assessment.

65 Other countries have tried this model - Romania being one example - and it usually ends up with very few projects being rejected as ministries trade favours.

66 According to the definition given at Article 4.11 of the PIL.

67 MOIT only has responsibility for heavy industry. 
Independent review needs strengthening so that an objective opinion on the quality of a feasibility study and the resulting appraisal findings is available to decision-makers.

128. A stronger independent review function is required in Vietnam to assure the quality of feasibility studies and counteract any optimism bias in cost and benefit estimates. Investment decisions are made by parties with a stake in the project, based on appraisals carried out by organizations/bodies that are not necessarily indifferent to the appraisal findings. The weakness of the current process is illustrated by the small proportion of projects that is rejected at appraisal/ investment decision stage.

129. The MPI is well positioned to lead the independent review of feasibility studies and appraisal findings from the perspective of social profitability. The administrative force of the independent review by the MPI, would need to be decided, but the exercise of a joint responsibility with the relevant technical line ministry seems the most appropriate path at this point in time. Independent review at this stage should extend beyond the narrow confines of affordability, to involve confirming the methodological quality of the feasibility study, the reliability of the underlying assumptions and forecasts, and the robustness of the appraisal findings. If the MPI were required to carry out independent review of all projects, its capacities would be stretched too thinly, so it would make sense to limit its review to Projects of National Importance and Group A. At local authority level, the province departments of planning and investment could fulfill the same function for Groups B and C. The MPI might use in-house expertise to review project proposals, contract out the work or use a combination of the two; the most important thing is its impartial status. 


\section{Summary Assessment of Independent Review}

\section{Key Functionality}

An independent peer review checks any subjective, self-serving bias in appraisal.

\section{Desirable Regulatory, \\ Administrative and Technical Arrangements}

1. Independent checks to ensure objectivity and quality of appraisals.

2. Disciplined completion of projects appraisals prior to budget preparation.

3. Identifying and maintaining an inventory of appraised projects ranked by priority for budgetary consideration.

4. Clarity of roles between projects that are minor and may be dealt with at the departmental level and those that require additional scrutiny.

\section{Current Arrangements in Vietnam}

Arrangements are now in place that give a degree of independence to appraisals, but these do not provide the level of impartiality required, leaving open the possibility of over-optimistic or low quality appraisals slipping through the net.

Consistent with 5-year medium-term investment plans, annual investment plans are prepared and approved according to a calendar set out in the PIL, which ensures completion before budget preparation begins. To be included in the annual plan, projects must be approved by the competent authorities, which requires an investment decision based on a feasibility study and appraisal findings.

There is no centralised electronic inventory of all projects that have received positive investment decisions and are eligible to be included in annual investment plans. This is partly a function of decentralised decision-making, but there is also no such inventory for central government projects.

The PIL incorporates differentiation of appraisal roles and decision rights by project importance. The MPI and MOF have no roles at this stage though, and there are some projects for which the level of central scrutiny is therefore inadequate.

\section{Recommendations}

\section{The diagnostic assessment above suggests the need to design and implement a strengthened independent review function in Vietnam, focusing on large-scale projects. The following specific actions concerning independent review are therefore recommended:}

- Develop and then apply a checklist of criteria for reviewing feasibility studies and appraisal findings.

- Amend the Construction Law and Public Investment Law to strengthen the role of the MPI in independent review, especially with respect to large-scale projects with significant fiscal impacts/A suitable allocation of responsibilities between the MPI and the leading technical line ministry should be ensured, with the former focusing on economic efficiency and strategic effectiveness.

- Assess capacity building requirements for MPI and MOC to perform an enhanced independent review role and undertake training.

- Develop a centralized database of projects, to assist the MPI in its independent review role both at appraisal and conception - and record the status of projects at different stages in the pre-implementation phase. 



\section{Selection and Budgeting}

\section{What Does Good Practice Look Like?}

131. There are four main components of good practice in selection and budgeting of public investment projects, as identified in the World Banks PIM framework:

- Once new projects are properly prepared and appraised, there need to be transparent criteria, which make reference to policy objectives, for selecting projects for inclusion in the budget.

- Existence of a well-structured budget preparation process with scope to integrate investment and recurrent implications of projects.

- Effective gatekeeping to ensure only appraised and approved projects are selected for budget financing.

- Ensuring adequate financing for selected projects, including recurrent needs on completion.

132. New projects must be verified at budget time as having been well prepared and carefully appraised. This is the 'gatekeeping' role. In weaker systems, inadequately prepared projects often slip through the net during budget preparation. Figure 10 illustrates the gatekeeping function, which sits between quality-at-entry systems ${ }^{68}$ and capital budgeting.

Figure 10: The Gatekeeping Function

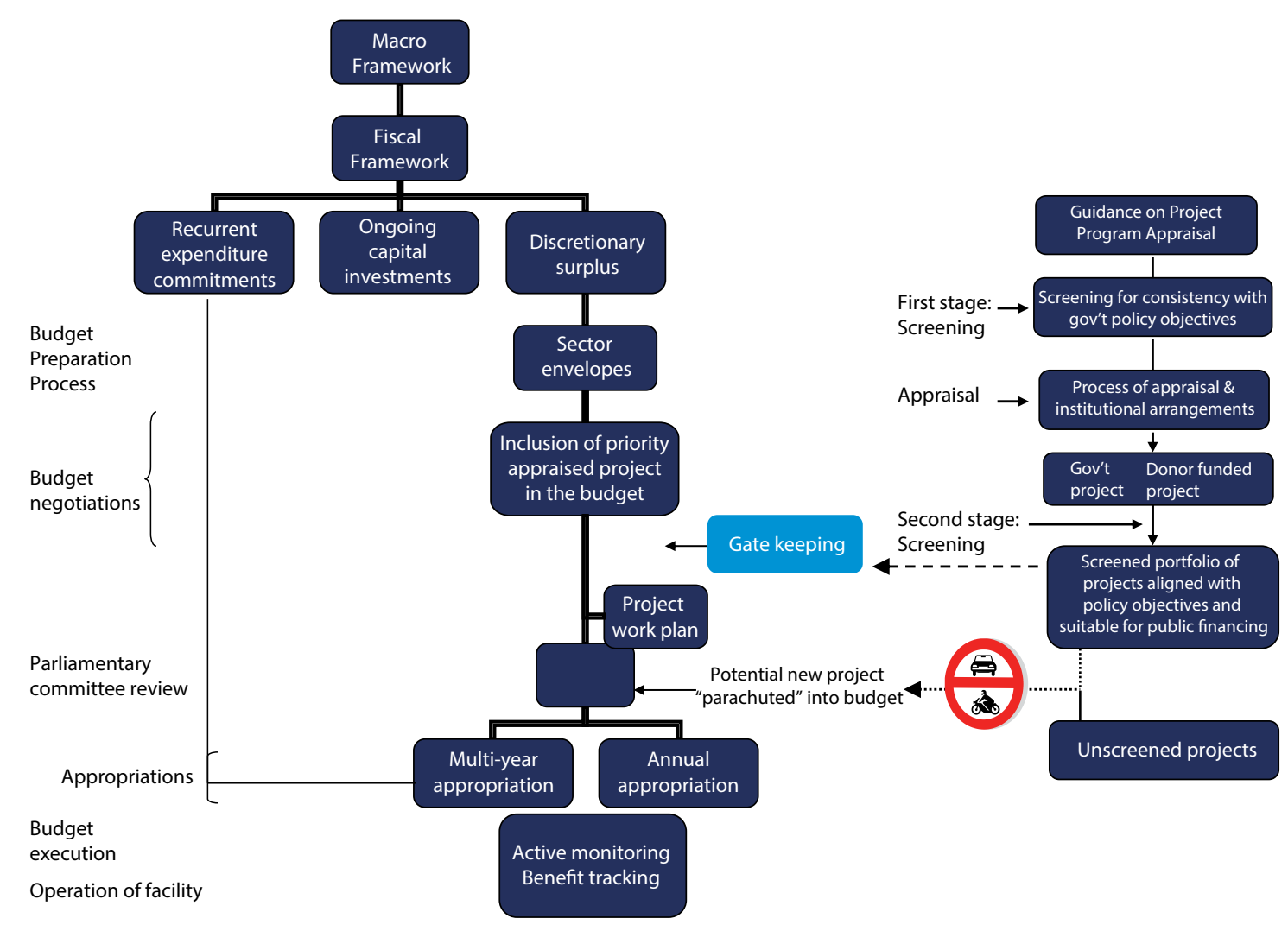

Source: A Diagnostic Framework for Assessing Public Investment Management, Anand Rajaram, Tuan Minh Le, Nataliya Biletska \& Jim Brumby, World Bank Policy Research Paper No. 5397, 2010.

68 The 'quality-at-entry' system involves the upstream processes of first-level screening, appraisal and independent review. 
133. Even in the best systems, there are likely to be more positively appraised new projects than there is funding and explicit prioritisation criteria must be applied reflecting national and sectoral priorities and practical factors, like readiness to go. In the more advanced systems, prioritisation of new projects is generally done by spending ministries within sector expenditure limits approved by the government on the basis of technical advice from the planning and finance ministry. Ideally, credible expenditure limits should be set for the medium-term to aid implementation planning for multi-year projects. Increasingly in certain good practice countries ${ }^{69}$, a stronger role in prioritising major or mega projects, i.e., projects of national significance, has been assumed by the Government, supported in this task by planning/finance ministries, rather than leaving prioritisation to individual ministries.

134. Major infrastructure projects usually take several years to complete, and efficient implementation demands that the implementing agency be able to enter into multi-year contracts. There should be confidence that budgetary resources will be made available in future years to meet these contractual commitments. This can be handled in various ways without undermining the annuality of the budget. Adequate funding for project preparation should also be assured through the budget and for major infrastructure projects this may extend over more than one budget year.

135. The capital budgeting process should be set up in such a way as to ensure efficient funding of on-going projects. In weaker systems, on-going projects compete directly against new projects for available financial resources, which often results in spreading funding across too many projects too thinly, compromising technically optimal implementation. In its extreme form, when there is overwhelming pressure from new projects, this can result in 'drip funding' of on-going projects, just sufficient to keep them notionally 'active'. The budget process should support, as much as possible, consistency in prioritisation over time ${ }^{70}$, which is best served by making a decision on funding for on-going projects before allocating funding to new projects. A frequent ploy of budget entities in less disciplined systems is to try to include new, 'stealth' projects in the budget. This involves having a small allocation approved in the first year that the project appears in the budget, only for funding requirements to balloon in subsequent years, thus squeezing out more mature projects under implementation. The introduction of a rolling medium-term budget planning perspective is one way of helping to prevent this.

136. Inadequate budgetary funding for the recurrent expenditure needs of newly completed capital investment projects is a frequent problem of less well-developed budgeting systems. The budgetary process must be sufficiently integrated to allow capital and recurrent expenditures to be planned together, so that budgetary provision is foreseen for the operating and maintenance expenditures of new facilities (e.g., staff salaries and medicines for new health facilities and routine and periodic maintenance costs for roads) when they come into service. This is often best done within a rolling medium-term horizon for expenditure planning.

69 These tend to be the countries where performance-based budgeting (PBB) has been introduced. PBB emphasises decentralised decision-making by line ministries, but in the case of capital spending, there has been a reversal of this trend, at least as far as major projects are concerned.

70 Meaning that, barring extreme, unforeseen circumstances or profound implementation problems, projects that were prioritised in previous periods should remain priorities and have first-call on funding until completed. 


\section{Assessment of Selection and Budgeting in Vietnam}

Criteria/principles for selecting projects for budget funding are defined in the State Budget Law and PIL, but they are not very precise, they lack a strong policy orientation, as far as selecting new projects is concerned, and their application is, in practice, not very transparent or consistent. While the criteria clearly give priority to on-going projects over new, their application implies a single decision-making process, when it is better to have a separation, first 'locking-in' funding for ongoing projects before attention turns to new projects, thus avoiding any possibility of trading off new against on-going projects.

137. Referring to the PIL for the details, the State Budget Law $\mathbf{2 0 1 5}$ gives only general guidance on budgeting for expenditure on public investment. The requirements for annual state budget estimates include the following stipulation:

Estimate of expenditure on development investment based on planning, plans, programs/projects approved by competent authorities; 5-year financial plans, plans for midterm investment of state budget, ability to balance resources in the budget year, regulations of law on public investment, construction, and relevant regulations of law;

Projects therefore need to have been approved by the competent authorities and to have been included in the medium-term investment plans for the state budget. Total expenditure estimates also need to fit within financial limits. The State Budget Law also assigns responsibility for preparing the central government capital budget to the Ministry of Planning and Investment ${ }^{71}$.

138. Capital budget proposals are based on annual investment plans which are, in turn, required to be in conformity with the approved MTIPs ${ }^{72}$, although some allowance is made for inclusion of emergency projects. Each ministry and central authority prepares an annual plan, as do local authorities. A consolidated national plan is also prepared. Annual plans include lists of projects and a budget plan, with proposed allocations to each project.

139. The PIL defines certain criteria for selecting projects for inclusion in the annual plan. Various principles, requirements and restrictions for preparing annual plans are set out in different places in the PIL (and would benefit from a clearer presentation). As a general principle, the PIL requires preference to be given to the projects with the most significant impact on socio-economic development. Among the more specific criteria, the following priority order for including projects in annual investment plans is established:

\section{- First priority:}

- Projects that have already been completed, but have outstanding payments;

- Projects that will be completed on schedule within the planning period;

- Counterpart funding for projects funded through ODA and international concessional loans;

- State participation in projects implemented through public-private partnerships.

71 Duties and entitlements of the MPI set out in the State Budget Law (Article 27) include:'Establish and propose principles, criteria, and limits on allocation of capital for investment in development of state budget to the government plan the allocation of expenditures on development investment of central government budget.'

72 Article 51.7 of the Public Investment Law 
- Second priority: Projects the implementation of which stretches across more than one five-year planning period, but which are being carried out according to the planned rate of progress.

- Third priority: New projects

140. While the prioritisation criteria in the PIL seem reasonable at first sight, on closer examination they do not offer strong enough safeguards for on-going projects. The first thing to note is that there is an explicit prioritisation of on-going projects over new projects. As a principle this makes sense, but it is best to embed it in capital budgeting processes - using a two-step approach as described below - and avoid altogether the possibility of making direct trade-offs. It is also notable that priority is given to projects where the state budget is leveraging other sources - ODA and PPP - which makes sense, provided that the reference is also to on-going projects. The second priority raises a question. It means that, for example, a very important mega project that will take more than 10 years to complete (and thus overlap five-year planning periods) will be given lower priority than a project that can be completed in 5 years. This is, in fact, a choice that should never really arise if good practices are already in place, which should ensure sustainable financing for both short and long duration projects. If such trade-offs are actually arising in practice in Vietnam, it supports the need for a portfolio clean-up (as discussed below) and the potential usefulness of a long-term, rolling infrastructure plan, as suggested earlier. It is notable that the PIL has no explicit requirement to include a priority ranking of those projects included in the annual plans. Finally, there are, in fact, no criteria specified for prioritising new projects.

141. The prioritisation criteria in the PIL do not have a strong policy orientation, beyond the broadly specified requirement for maximising socio-economic development impact. This is presumably not seen to be necessary because the projects that end up in the annual plans originate from the MTIPs, which should have been elaborated as instruments for achieving the policy priorities expressed in the socio-economic development plans and strategy. If the planning system works as intended, projects that arrive in the annual investment plan should, therefore, already be closely aligned to strategic policy priorities. This is a strong assumption. As has been discussed in the section on strategic guidance, while the MTIPs have introduced more realism into financial programming of investment, they still tend to be over-ambitious, meaning that they contain more projects than can realistically be implemented over their five-year term. The realism of the SEDPs, which form the basis for elaborating the MTIPS, is also questionable, and the calendar for preparation of the SEDP and MTIP has not, in practice, been properly harmonised, so far. MTIPs can also pull in projects from multiple planning instruments, beyond the SEDPs, diluting their strategic focus. All this points to a need for a further layer of prioritisation, on top of the process that yielded the MTIPs, when selecting projects for inclusion in annual investment plans. The latter cannot be expected to drop automatically out of the MTIPs. Box 14 gives an advanced country example of a harmonised system of strategic planning, appraisal and budgeting. 


\section{Box 14: Linking Strategic Planning, Project Appraisal and Budgeting in the Netherlands}

In the Netherlands, the National Policy Strategy for Infrastructure and Spatial Planning (SVIR), a comprehensive vision for the country to 2040 with goals to 2028. The SVIR is implemented through the Multi-Year Plan for Infrastructure, Spatial Planning and Transport (MIRT).

The MIRT is both a medium-term investment plan (the 'MIRT Project Book') and a set of rules (the 'MIRT Rules') for project development and implementation. The MIRT Project Book is the medium-term investment programme of the Government which is rolled over annually. It brings together national investment and local/regional investment.

The MIRT Project Book is annexed to the annual budget and presents projects to be funded in line with the priorities set out in the SVIR and the Government Programme. The Project Book, which is available online, explains the basis for selection, referring to the focal areas for central government action identified in the SVIR

Source: 'Study on Cross-Country Comparison', Technical Assistance for Public Investment Project Appraisal and Selection System Development in Ukraine, World Bank, 2014.

142. The task of selecting projects for budget funding becomes more manageable when preceding stages of the PIM system are working effectively and filtering out weak projects (see Figure 10). When the quality-at-entry system and gatekeeping function (see below) are working effectively, only projects which have been confirmed as representing a good use of public financial resources ought to be presented for budget funding. While this also means that appraisal findings will generally have less relevance than other factors when budgeting ${ }^{73}$, it does not mean that they are irrelevant. When all other factors are equal, it does make sense to focus public financing on those projects which bring a higher social return for a given expenditure; however, other factors are usually not equal when it comes to these budgetary decisions and a host of other factors usually intervene ${ }^{74}$.

\section{The kind of criteria that should generally guide capital budgeting decisions for new projects are:}

- Strategic importance of the project and the sector based on government policy as expressed in strategic planning documentation and the budget strategy paper (or its equivalent);

- Significance and sustainability of future budgetary consequences on completion (operating and maintenance costs);

- Compatibility with other new projects and non-capital expenditure proposals;

- Readiness to go in the forthcoming budget year, including deliverability of plans for detailed design and procurement;

- Compatibility with the spending ministry's (or department's) portfolio of on-going projects in terms of implementation capacity; and

- Impact on the overall balance and risk profile of the sector and national public investment program.

Regardless of possible areas for refining the existing selection criteria, it is important not to expect too much of economic analysis methods when it comes to budgeting and inter-sectoral prioritization, as discussed in Box 15.

73 Except to the extent that they will have been instrumental in determining a project's eligibility to be presented for budgeting.

74 "Final prioritisation also [reflects] other factors, including the government's political and strategic priorities, deliverability, commitments and regional distribution": 'Managing Budgeting in Government', the UK National Audit Office's 2012 summing up of the expenditure decisions made in the 2010 Spending Review. 


\section{Box 15: Limitations of Economic Analysis Methods for Selection and Budgeting}

SCBA is useful in quality controlling projects and ensuring that those projects that go forward into the budget arbitrage are socially profitable and sustainable. It is also a useful aid to prioritising projects within sectors/sub-sectors, roads or irrigation, for example. However, even if it could be applied to all projects, which it cannot, SCBA is not a universal prioritisation tool capable of providing a scientific ranking of all projects across all sectors as a basis for budgeting. The relative importance of non-monetised benefits and costs differs between sectors (and even sub-sectors), as does the reliability of benefit estimation techniques, making it difficult to make cross-sector comparisons. And in those sectors where SCBA is not readily applicable, because of difficulties in valuing benefits, CEA does not produce an absolute indicator of net project worth for comparison with other projects. In fact, there are no examples of good practice countries where economic appraisal findings are used as the main means of determining budget priorities, although these can form one piece of information in the decision-making process. Faced with the limitations of economic analysis in capital budgeting, multi-criteria scoring systems look superficially appealing as an alternative 'scientific' approach to ranking projects across sectors and arriving at a prioritised budget. However, before embarking on this path it is worth noting that few if any countries use such an approach for budgeting, and certainly no countries regarded as having good practices. Universal ranking systems, while appealing to technicians, ignore the political dimension to budgeting. The PIM system should ensure that the space within which political decisions are taken is narrowed down to a set of quality-controlled projects and that there is an inter-temporal consistency to decisions, i.e., on-going projects are fully funded, before new projects are considered.

Source: 'Strengthening Capital Budgeting in Georgia' Unpublished Policy Note for PER, World Bank 2010.

Vietnam has recently streamlined its annual budget preparation process and introduced the concept of the rolling, three-year budgetary perspective. This is a potentially important improvement for PIM, but detailed design is still on-going and full implementation some way off, so the impact is yet to be felt.

144. The State Budget Law 2015 introduced the requirement for three-year 'state budgetfinance plans' to be developed at national and province levels, offering the potential for a significantly improved budgetary environment for PIM. A medium-term perspective for fiscal and expenditure planning is important for PIM because: i) implementation of projects usually lasts longer than one budget cycle; ii) projects have future expenditure consequences once construction is completed; and iii) strategic investment planning, prioritisation and decision-making require a longer term view of resource availability than is provided by the annual budget. The advantages of a rolling, medium-term budgetary perspective for $\mathrm{PIM}^{75}$ are therefore that it:

- Allows the planning of expenditures for efficient implementation of on-going multi-year projects;

- Assists in estimating the sustainable level of public financing for new spending initiatives, including for new projects;

- Allows expenditure programming over a multi-year perspective consistent with planning horizons adopted in strategic planning guidance;

- Provides a realistic and supportive framework for strategic resource allocation decisions, including resource allocation for capital investment expenditure;

75 Notwithstanding these advantages, a three to five year perspective will often be too short to encompass the planning, implementation and commencement of operations of the largest projects. As indicated in the earlier section on strategic guidance, supplementary, longer-term, rolling strategic expenditure plans may therefore be helpful for national infrastructure or for important infrastructure sectors. 
- Creates a conducive incentive environment for prioritization of new projects in line with strategic planning guidance; and

- Allows planning for the recurrent expenditure requirements of capital investment projects upon completion.

145. A functioning medium-term expenditure framework (MTEF) is an essential component for effective PIM, allowing the proper planning of multi-year expenditure commitments and linking fiscally sustainable medium-term capital budget allocations to strategic plans. Design and implementation of Vietnam's medium-term budgetary perspective is still on-going, leaving room for the authorities to ensure that its final form can serve to strengthen the PIM system. A medium-term fiscal framework (MTFF) is a basic condition for a minimally functional budgetary system. Vietnam currently has a static five-year fiscal framework as a basis for five-year planning (SEDPs and MTIPs), and a rolling three-year fiscal framework within which actual annual budget allocations are planned and approved/The latter is closer to the concept of an MTFF. A medium-term budget framework (MTBF) is the next stage of MTEF development and is a prerequisite for achieving more efficient strategic resource allocation of budgetary resources for both recurrent and capital expenditure. This is where Vietnam is now, and the next steps will be important for strengthening PIM. PIM can be further enhanced by introducing a stronger performance orientation into the MTEF, but this is not seen as an immediate priority for Vietnam, at the present time, and is probably best seen as a longer-term objective. As an example of international experience in MTEF development, Box 16 illustrates the evolution of a medium-term budgetary perspective in Ireland, with a focus on capital investment. 


\section{Box 16: Capital Budgeting in a Medium-Term Perspective in Ireland}

Ireland's 2004 Budget introduced rolling multi-annual envelopes for capital expenditure for the first time. An envelope is a binding aggregate expenditure allocation, i.e., not defined by project, which is valid for an extended period of time and which cannot be exceeded. This provides a stable and predictable financial framework within which multi-year projects can be planned, programmed and implemented.

The envelopes are set for five years and are rolled forward annually by one year. The aggregate capital allocation is broken down by ministry ('department') or other relevant higher-order spending agency. The envelope is allocated by year over the five-year period, but the possibility exists to carryover unused allocations subject to certain restrictions. Spending ministries must plan and implement their capital expenditure within the limits set by the envelopes.

In the case of capital investment in transport infrastructure, the Irish Government decided in November 2005 to go further and to provide for a ten-year multi-annual envelope - called Transport 21 - to take account of the longer time-scale for planning and implementing major transport infrastructure projects, and to tackle the transport infrastructure deficit. Transport 21 was not, however, a rolling framework and covered the fixed period 2006-2015. It was subsequently pushed off-track when the global financial crisis engulfed the country.

There is an unallocated reserve providing additional amounts which are available for allocation each year (except the first year) to support new Government priorities for capital investment. Within the aggregate allocation for capital expenditure for 2004-2008, this represented around $7 \%$ of the total. The unallocated reserve is not to meet price and physical contingencies arising from planned and on-going projects: ministries must plan for such contingencies within their allocated capital envelopes.

As part of the Comprehensive Review of Expenditure 2012 - 2014, Ireland introduced a medium-term expenditure framework (MTEF) with binding medium-term financial constraints for most of current and all of capital expenditure for a three year period. The ceilings for current expenditure do not apply to demand driven expenditures (unemployment benefits for example), which are still planned and managed on an annual basis. This is similar to the UK model.

The aggregate expenditure ceilings for the MTEF are set by the Government upon the recommendation of the Minister of Finance who consults with the Minister for Public Expenditure and Reform. The allocations between ministries ('ministerial ceilings') are set by the Government upon the recommendation of the Minister for Public Expenditure and Reform. Having regard to fiscal and economic conditions, the Minister of Finance makes a recommendation to the Minister for Public Expenditure and Reform on the proportions of total expenditure that should go to capital and current expenditure.

Source: 'Study on Cross-Country Comparison', Technical Assistance for Public Investment

146. Reconciling the static planning horizons of investment planning instruments with the rolling horizon of the 'state budget finance-plans' raises issues that will need to be addressed during the design of Vietnam's MTEF. Annual investment plans are supposed to be based on the MTIPs, which cover fixed five-year planning periods. The financial resource framework - with which the MTIPs are intended to be consistent - also covers the same five-year period. The 'state budget financeplan' is a rolling, three-year framework that adapts to new information and changes in macro-fiscal circumstances and, as such, it is a more useful tool for the financial programming of public investment. A divergence between the expenditure foreseen in the MTIPs and what is achievable through the 'state-budget finance-plan' is highly likely to emerge and get worse towards the end of the static planning period. In these circumstances, should annual investment plans stick rigidly to the MTIP or adapt to the fiscal realities? If the latter, which seems justifiable, then should there also be rolling, three-year public investment programmes aligned with the state-budget finance-plan? Again this seems like a sensible idea, but risks undermining the status of the MTIPs, which have a stronger legal standing, since they are approved by the National Assembly. 
147. The timetable for preparation of annual investment plans in the PIL is harmonised with the budget preparation calendar in the SBL, but there may be a need for earlier activities if preparation of a capital baseline (discussed below) is adopted as part of the MTEF design. Budget preparation begins on 15 May when the Prime Minister issues regulations on preparation of annual socio-economic development plans and state budget estimates. Budget preparation is completed on the part of the executive side of government when the draft budget documentation is submitted to the Standing Committee of the National Assembly on 20 September (although revisions may follow on the basis of comments). Between these dates, the PIL foresees the drafting of annual investment plans at the level of ministries, regulatory authorities and local authorities These are to be submitted to their respective higher level planning authorities (MPI and DPI) for assessment and redrafting within financial limits established by the financial authorities (MOF and DOF), before resubmission to the MPI by 10 September for consolidation. This whole process begins with the issuing of instructions by the MPI on 15 June and culminates with the submission of the national annual investment plan by the MPI to the Government before 20 September. Deriving from Vietnam's decision to adopt an MTEF-style instrument (three-year state budget finance plans), there are a number of considerations in relation to this process which will probably need to be addressed:

- The calendar for preparing the draft budget and draft annual investment plans is tight, and leaves little room for developing a strategic medium-term budgetary perspective as a precursor to preparation of the detailed annual budget. Depending on the design adopted, MTEF preparation may well require activities to take place before 15 May and, if this is the case, this also implies earlier work on annual investment plans than is currently foreseen in the PIL.

- Again depending on the MTEF design, annual investment plans will probably need to be accompanied by a medium-term (three-year) perspective, which is not foreseen in the PIL. (The challenge of reconciling these with the static, five year perspective of the MTIP has already been mentioned).

- Whatever the MTEF design, there will be an enormous benefit from preparing forward estimates of the expenditure requirements for on-going projects (the 'capital baseline') before 15 May, so that these can then inform the budget preparation process that follows. A mediumterm budgeting system requires that the no-policy-change baseline expenditure estimates [forward estimates] be updated right at the start of the budget preparation process76. These forward estimates should look beyond the forthcoming budget year in preparation. This is as foreseen in the implementing regulations for the SBL, and also needs to be reflected in a future revision of the PIL, so as to ensure consistency.

Vietnam suffers from poor integration of the capital and recurrent budgets, which are prepared separately by different institutions.

148. Vietnam has a dual budgeting system, whereby the MPI is responsible for preparing the capital budget and the MOF is responsible for preparing the recurrent budget. The separate budgeting processes lead to poor integration between capital and recurrent expenditure, which manifests itself in a number of ways, but most notably in the underfunding of the maintenance of capital assets. The comprehensive approach to expenditure planning that forms part of the MTEF approach, together with the perspective beyond the annual budget, present an opportunity for a more integrated approach to budgeting. This opens up the potential for a more coordination in allocating funding for the maintenance and operation of existing and new fixed assets. The separate budgets

76 This applies of course to both capital spending and recurrent spending. 
and divided responsibilities have, nevertheless, been reaffirmed in the State Budget Law 2015 and the realisation of a more integrated approach to budgeting will depend critically on the detailed design of Vietnam's MTEF (three year 'state budget-finance plan') and on enhanced coordination between the MPI and the MOF within this framework.

As in many countries where more advanced capital budgeting processes are still in their formative stages, Vietnam suffers from too many inadequately funded on-going projects, resulting in completion delays, postponed delivery of expected benefits and lower social profitability.

149. The evidence is that many projects take longer to implement than planned because of budgetary shortfalls. For projects funded from the state budget, either at central level or province level, but especially the latter, 'drip-funding' of too many projects is frequent, leading to delayed and lower impacts than foreseen during planning. Evidence for this can be seen among the sample of education projects surveyed for this assessment, with a number experiencing extended implementation periods because if shortage of funding for efficient execution. These projects will generally be those falling into Groups B and C. This is not the case for the sample of transport projects ${ }^{77}$ - see Table 9 - where the majority reports adequate budgetary allocations for efficient implementation and no build-up of arrears. While these projects have earmarked funding from ODA or government bonds, some of them did not have adequate counterpart funding or suffered from the consolidation of public investment, implemented with Directive 1792 from the end of $2011^{78}$.

Table 9: Adequacy of Budget Funding for Sampled Transport Projects

\begin{tabular}{|c|c|c|c|c|c|}
\hline \multirow[b]{2}{*}{ Project } & \multirow[b]{2}{*}{$\begin{array}{l}\text { Main Funding } \\
\text { Source: } \\
\text { ODA } \\
\text { Bond } \\
\text { State Budget }\end{array}$} & \multicolumn{2}{|c|}{$\begin{array}{l}\text { Ensuring adequate financing for } \\
\text { selected projects }\end{array}$} & \multicolumn{2}{|c|}{$\begin{array}{l}\text { Timeliness and predictability of } \\
\text { in-year funding }\end{array}$} \\
\hline & & $\begin{array}{l}\text { Q45: Has the } \\
\text { amount budgeted } \\
\text { for the project } \\
\text { ever been below } \\
\text { the amount } \\
\text { initially planned } \\
\text { for technically } \\
\text { optimal } \\
\text { implementation? }\end{array}$ & $\begin{array}{l}\text { Q46: Has there } \\
\text { been any arrears } \\
\text { accumulated } \\
\text { during project } \\
\text { implementation? }\end{array}$ & $\begin{array}{l}\text { Q43: Have } \\
\text { there been } \\
\text { any problems } \\
\text { concerning } \\
\text { the reliability } \\
\text { of the flow of } \\
\text { funds during } \\
\text { the budget } \\
\text { year? }\end{array}$ & $\begin{array}{l}\text { Q44: Has there } \\
\text { ever been a } \\
\text { shortfall in } \\
\text { funding at } \\
\text { the end of the } \\
\text { budget year } \\
\text { compared } \\
\text { to the initial } \\
\text { budget } \\
\text { allocation? }\end{array}$ \\
\hline \multicolumn{6}{|l|}{ Transport Projects } \\
\hline Hung Ha Bridge & ODA & (Yes) & Yes & No & No \\
\hline $\begin{array}{l}\text { Hanoi-HCMC Railway } \\
\text { Bridges }\end{array}$ & ODA & No & No & No & No \\
\hline $\begin{array}{l}\text { Da Nang-Quang } \\
\text { Ngai Expressway }\end{array}$ & ODA & No & Yes & Yes & No \\
\hline Terminal 2 Noi Bai & ODA & $\mathrm{n} / \mathrm{a}$ & $\mathrm{n} / \mathrm{a}$ & $\mathrm{n} / \mathrm{a}$ & $\mathrm{n} / \mathrm{a}$ \\
\hline $\begin{array}{l}\text { Nghi Son - HCMC } \\
\text { Road }\end{array}$ & State budget & No & No & No & No \\
\hline
\end{tabular}

77 Evidence from the education projects is less easy to interpret, as there were no responses to the relevant questions in two cases and the projects were too recently started for the questions to apply in another two cases. For the rest there seem to have been no funding shortfalls.

78 Another problem relates to inadequate allocation of resources when project are implemented faster than planned, but this is an issue of in-year budget adjustments, which is dealt with under implementation. 


\begin{tabular}{|c|c|c|c|c|c|}
\hline \multirow[b]{2}{*}{ Project } & \multirow[b]{2}{*}{$\begin{array}{l}\text { Main Funding } \\
\text { Source: } \\
\text { ODA } \\
\text { Bond } \\
\text { State Budget }\end{array}$} & \multicolumn{2}{|c|}{$\begin{array}{l}\text { Ensuring adequate financing for } \\
\text { selected projects }\end{array}$} & \multicolumn{2}{|c|}{$\begin{array}{l}\text { Timeliness and predictability of } \\
\text { in-year funding }\end{array}$} \\
\hline & & $\begin{array}{l}\text { Q45: Has the } \\
\text { amount budgeted } \\
\text { for the project } \\
\text { ever been below } \\
\text { the amount } \\
\text { initially planned } \\
\text { for technically } \\
\text { optimal } \\
\text { implementation? }\end{array}$ & $\begin{array}{l}\text { Q46: Has there } \\
\text { been any arrears } \\
\text { accumulated } \\
\text { during project } \\
\text { implementation? }\end{array}$ & $\begin{array}{l}\text { Q43: Have } \\
\text { there been } \\
\text { any problems } \\
\text { concerning } \\
\text { the reliability } \\
\text { of the flow of } \\
\text { funds during } \\
\text { the budget } \\
\text { year? }\end{array}$ & $\begin{array}{l}\text { Q44: Has there } \\
\text { ever been a } \\
\text { shortfall in } \\
\text { funding at } \\
\text { the end of the } \\
\text { budget year } \\
\text { compared } \\
\text { to the initial } \\
\text { budget } \\
\text { allocation? }\end{array}$ \\
\hline $\begin{array}{l}\text { Moi Market-Chu } \\
\text { Market Road }\end{array}$ & Bond & No & No & No & No \\
\hline $\begin{array}{l}\text { Mekong Delta } \\
\text { Transport } \\
\text { Infrastructure }\end{array}$ & ODA & No & No & Yes & Yes \\
\hline $\begin{array}{l}\text { National Highway } \\
\text { No. } 1 \text { Extension }\end{array}$ & Bond & No & No & No & Yes \\
\hline $\begin{array}{l}\text { Hau River Navigation } \\
\text { Channels }\end{array}$ & Bond & No & No & Yes & Yes \\
\hline Bim Son Bridge & Bond & No & No & No & No \\
\hline
\end{tabular}

150. Appropriately designed budgeting processes are the best way of ensuring that funding for new projects is not at the expense of optimal funding for on-going projects. As already discussed, the PIL indicates that (problem-free) on-going projects should be given priority over new projects when selecting projects for inclusion in the annual investment plan. This is common sense, but not so easy to achieve in practice, especially if processes are unsupportive. The least effective approach is to undertake a simultaneous prioritisation of on-going and new projects: despite exhortations to the contrary, on-going projects are more than likely to lose out in the trade-off, which will inevitably involve pressure from the political level. This seems to be what happens in Vietnam at the moment, particularly at province level, leading to on-going projects losing out to new projects.

151. Introducing a two-step ceiling setting process as part of the MTEF, could help guard against the problem of underfunding of on-going projects ${ }^{79}$. Such a process involves first making projections of expenditure requirements for continuation of existing expenditure policies before allocating discretionary fiscal space to new spending initiatives. As far as capital expenditure is concerned, this means establishing the expenditure requirements for efficient completion of ongoing or committed projects - the 'capital baseline' - before making decisions on budgetary allocations for new projects. This serves four purposes:

- It establishes minimum expenditure requirements for on-going projects, thus setting a lower boundary for ceilings; ${ }^{80}$

79 This does not mean that decisions subsequently revealed as poor uses of public finances should be continued, no matter what: governments should always be ready to stop wasteful projects.

80 This is when the system is stable and has been functioning well. If this is not the case, the capital baseline may not be fundable and a rationalisation of the national portfolio of public investment projects will be required before attention to the capital baseline will yield results. 
- It reduces the potential for competition over available financing between on-going and new projects by directing budgetary discussions towards the allocation of the genuinely discretionary fiscal space available for new capital projects;

- It provides information on how investment expenditure ceilings may have to be adjusted between line ministries to accommodate major new projects considered as priorities ${ }^{81}$, while assuring efficient funding for on-going projects; and

- It helps in programming the efficient implementation of new projects over the medium-term, so that there will be no funding shortfalls.

Figure 11 illustrates the concepts discussed above. The fiscal space for new projects is shown as the difference between the total resources available for public investment, minus a contingency, and the capital baseline. As illustrated, available fiscal space for capital spending can be expected to increase over time as on-going projects are completed and financial resources are freed up for new projects. It thus becomes easier to allocate funding to new projects towards the end of the medium-term horizon. Figure 11 should be interpreted as applying at the aggregate level, with the resource envelope for public investment derived from the MTFF and reflecting government preferences for capital over current spending.

152. A two-step process should also be adopted internally by line ministries and central agencies (and province departments for province MTEFs) when deciding how to allocate their designated capital expenditure ceilings. Financial resources should first be allocated for their capital baselines before introducing new project proposals, while remaining within ceilings. Right at the beginning of the first phase of the MTEF/budget preparation process, line ministries and central agencies (and province departments for province MTEFs) should be required to prepare baseline estimates for capital expenditure ${ }^{82}$, showing the forward funding implications of on-going and already committed projects. These should be based on the latest monitoring information, procurement plans and the financial plans for projects, updated as necessary.

Figure 11: Capital Baseline and Fiscal Space for Public Investment Projects

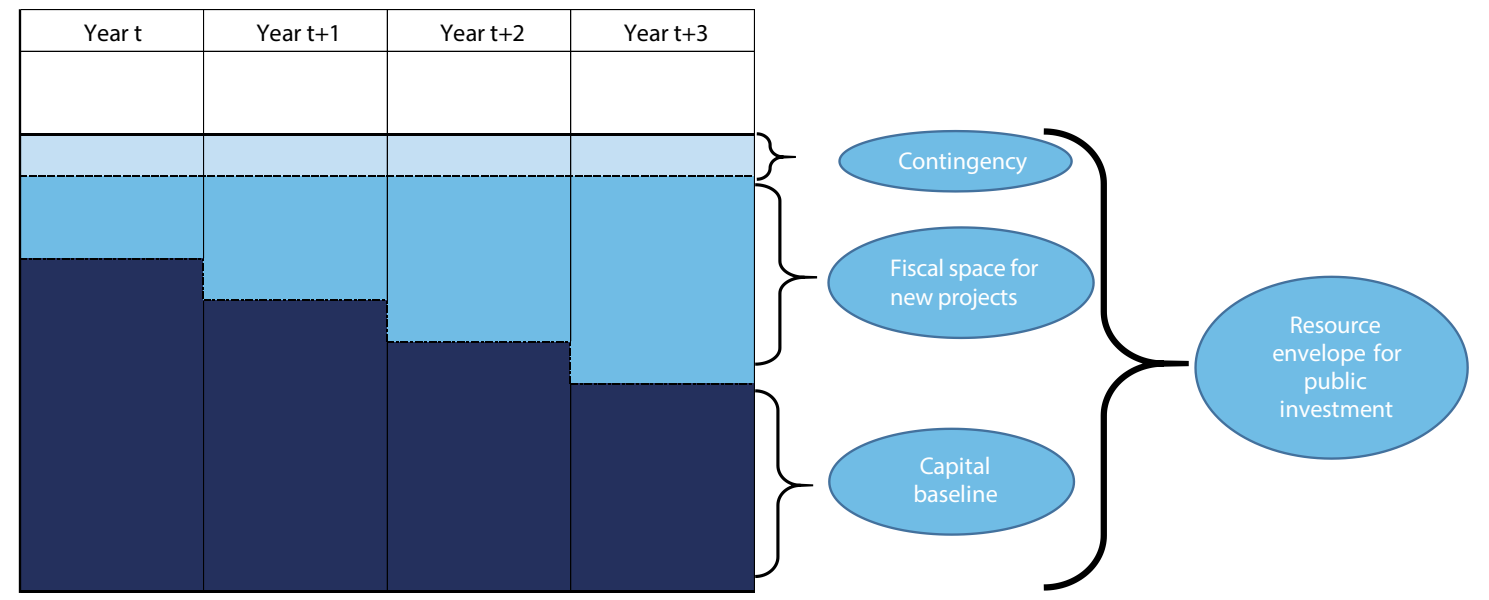

Note: The figure illustrates a 4-year perspective, but the idea applies equally to a 3-year or 5-year perspective.

81 Because of the scale and indivisibility of major capital projects, step-changes in ceilings will generally be required to accommodate new projects. Generally, such step-changes can be more readily accommodated in the final year of the MTEF horizon, which will not have been included in the previous year's MTEF.

82 Line ministries should also prepare baseline estimates for current expenditure. 
153. In the two-step ceiling setting process, the MPI (or DPI for provinces) should verify independently the coherence and realism of the line ministries' (departments') forward financing estimates for on-going and already committed capital investment projects. Ideally, these estimates should then be agreed with the respective ministries before any discussion of allocations for new projects. The MPI would then consolidate the agreed estimates to arrive at aggregate forward capital estimates. These will then serve as a basis for determining the aggregate discretionary fiscal space available for capital spending and for setting provisional capital expenditure ceilings for individual ministries. In this process, there is no explicit prioritization of on-going projects over new projects, because funding for on-going projects is 'locked in' before attention turns to prioritizing new projects within the available fiscal space.

154. The two-step process cannot be introduced without first undertaking a portfolio cleanup. If the funding requirements for efficient implementation of on-going projects exceed the total financial envelope for capital expenditure, because of past failings in controlling the entry of new projects into the portfolio, then the capital baseline is unsustainable. This seems to be the case in Vietnam, as mentioned previously, with the problem being focused on projects in the Group B and $C$ categories and at province levele ${ }^{83}$. In this case, there needs to be a restructuring of the portfolio of on-going projects, to remove those that have been stalled or slow moving for too long. Such projects should be removed forever where they are no longer strategically relevant, or should re-enter the PIM system at the beginning, to be re-assessed just as any new project concept. A portfolio clean-up will be a sensitive exercise and is only feasible if championed the highest levels of the leadership. It is nevertheless critical for the establishment of a process for ensuring adequate funding for selected projects.

Vietnam has no designated 'gatekeeper' to prevent non-compliant projects from entering the capital budget.

155. The PIL and the Construction Law set out Vietnam's quality-at-entry processes, but no agency is formally assigned the task of ensuring that projects proposed for budget funding are eligible. Capital budgeting must respect the quality-at-entry processes by preventing projects from being 'parachuted' into the budget without first having been appraised and selected as 'budgeteligible.' The absence of a strong 'gatekeeping' function to enforce quality-at-entry decisions is a frequent problem with less well-regulated PIM systems. New laws and regulations have introduced much greater discipline into Vietnam's system and reduced the risk of project entering the state budget by the backdoor, nevertheless without a formalized verification this is still a risk, particularly in relation to Group B and C projects and projects claiming to be a response to 'emergencies'. Gatekeeping is an administrative check on projects presented in budget requests to ensure compliance. It is not the same as independent review, which is more of a challenge function that should precede budgeting. Gatekeeping is usually performed by the ministry responsible for preparing the capital budget and relies on this ministry having sufficient power to authority to turn away non-compliant projects.

156. The highly regulated project preparation process, combined with onerous sanctions for irregularities, ensures that it is unlikely that a project would be able to 'jump the fence' into the budget, although there are some exceptions. Neither the MPI nor the MOF performs a distinct'gatekeeping' role, whereby projects are verified to ensure that they have been properly 'quality controlled'

83 As the sample survey of transport projects illustrates, major projects with earmarked funding - ODA and bonds - do not suffer from this problem. 
according to the pre-investment procedures in place ${ }^{84}$. The presumption is that budget entities will have done what is necessary before submitting funding requests and the information submitted at this stage is, anyway, insufficient for any sort of in-depth review.

157. There will inevitably be pressures to exempt certain projects from the PIM system, usually because of their supposed 'urgency'. The PIL leaves the door open quite wide by defining emergency projects as being 'aimed at timely overcoming of natural disasters and other unexpected circumstances ${ }^{\prime 85}$. International experience is that if such a fast-track exists, it will probably be overexploited, particularly in relation to politically driven projects, unless adequate safeguards are in place. This means being very specific about the circumstances when a project may be fast-tracked and establishing a clear set of procedures for determining eligibility. Exemptions must only relate to unforeseeable events and should take in such things as natural disasters, humanitarian emergencies and emerging national security threats. The final decision on eligibility is best taken outside the political arena, to avoid political 'horse-trading. Even for projects that are exempted, there needs to be a minimum of analysis performed and documentation prepared, so as to ensure an adequate and proportionate response to the new problem.

\section{Climate change adaptation does not yet play a part in prioritisation of projects for budget funding in Vietnam.}

158. The climate change adaptation framework has not yet been mainstreamed in Vietnam. Evidence from the sample of transport projects (see Table 8 in Section 6) shows that the climate change adaptation framework was not used in prioritising any of the sample of transport projects for budget funding. For five out of 10 projects the response was negative. In two cases, the question seems to have been confused with MONRE's role in reviewing EIAs ${ }^{86}$, but it can be assumed that the adaptation framework was not applied. No responses were given in three cases. While the adaptation framework does not feature in expenditure decision-making, budget allocations do, in the vast majority of cases, include funding for complementary measures to make project more sustainable and/or resilient, and a contingency for unforeseen circumstances, including climate-related events.

84 The usefulness of a formal gatekeeping function is also in doubt when there are questions about the completeness of the methodological basis for project appraisal (see Section 4).

85 Article 4.14 of the Public Investment Law

86 MONRE has no role in prioritising transport projects for budget funding. 


\section{Summary Assessment of Project Selection and Budgeting}

\section{Key Functionality}

The process of
appraising and
selecting public
investment projects is
linked appropriately to
the budget cycle.

The process of appraising and linked appropriately to the budget cycle.

\section{Desirable Regulatory, \\ Administrative and Technical Arrangements}

5. Transparent criteria for selecting projects with reference to policy objectives.

\section{Well-structured} budget preparation process with adequate provision for multi-year projects and scope to integrate investment and recurrent implications of projects.
There are defined criteria - 'principles' - for selecting projects for inclusion in the annual investment plans that provide the detail of the capital budget; however, the criteria do not cover all the expected dimensions and are not policy oriented. The weak policy orientation derives from the structured strategic planning process already underpinning the preparation of mediumterm investment plans; however, the planning process is not yet very discriminating, so that these plans are over-ambitious, indicating the need for a further filter to select projects for funding. Prioritisation of ongoing over new projects is one of the criteria; this is a valid objective, but might be better achieved by reengineering processes.

The 2015 State Budget Law brought significant improvements to Vietnam's budget preparation processes, but one of the most important changes for PIM purposes, the introduction of a rolling mediumterm budgetary perspective, is not yet fully functioning. If designed carefully, it could greatly improve the financial programming of multi-year projects and help in ensuring adequate funding for on-going projects, a major cause of delay and diminished development performance.

Reconciling the rolling, three-year horizon of the medium-term budgetary perspective with the static, five-year horizon of the socio-economic development plans and with the linked medium-term investment plans presents problems and will have to be addressed during design.

Vietnam has separate capital and recurrent budgets prepared by different ministries. Integration of the investment and recurrent implications of projects is made difficult by this arrangement (which is replicated internally within spending organisations) and leads to frequent underfunding of recurrent expenditure requirements, especially maintenance.

7. Effective gatekeeping to ensure only appraised and approved projects are selected for budget financing.
Under the new PIL, there are strict rules about the documentation and approvals required for projects to be eligible for consideration for funding through annual investment plans; these will help to ensure that only compliant projects are considered for funding. No organisation is formally nominated as 'gatekeeper' to ensure that non-compliant projects do not enter budget proposals, and the definition of emergency projects, which can circumvent upstream PIM procedures, is too loose, leaving room for some projects to enter the budget through the 'backdoor'. 


\begin{tabular}{|c|c|c|}
\hline Key Functionality & $\begin{array}{l}\text { Desirable Regulatory, } \\
\text { Administrative and } \\
\text { Technical Arrangements }\end{array}$ & Current Arrangements in Vietnam \\
\hline & $\begin{array}{l}\text { 8. Ensuring adequate } \\
\text { financing for selected } \\
\text { projects, including } \\
\text { recurrent needs on } \\
\text { completion. }\end{array}$ & $\begin{array}{l}\text { The PIL requires that priority be given to completion } \\
\text { of well-performing on-going projects and that full } \\
\text { provision is made for the funding of new projects over } \\
\text { their implementation periods. These are important } \\
\text { provisions, but processes do not support their } \\
\text { implementation yet. The introduction of the medium- } \\
\text { term budget perspective could eventually help if } \\
\text { the design includes a stepped process, which locks } \\
\text { in funding for on-going projects before attention } \\
\text { turns to new projects. Such a design forms part of the } \\
\text { implementing regulations of the SBL, but will also need } \\
\text { to be reflected in the PIL. } \\
\text { Little attention is paid to future recurrent financing } \\
\text { needs when a project first enters the budget. Only } \\
\text { in the year of commissioning is consideration then } \\
\text { given to the financing of operating and maintenance } \\
\text { expenditures, which is generally too late to ensure } \\
\text { adequate provision without squeezing other recurrent } \\
\text { expenditures. The medium-term budget perspective, } \\
\text { which is being introduced, provides a framework for } \\
\text { addressing this issue but, in the context of dual budgets, } \\
\text { will still require greater coordination between the lead } \\
\text { planning and finance organisations at different levels, if } \\
\text { it is to work effectively. }\end{array}$ \\
\hline
\end{tabular}

\section{Recommendations}

159. Three headline recommendations follow from the analysis of selection and budgeting. Firstly, project selection and budgeting need to be situated in a realistic medium-term, rolling budget perspective. Secondly, capital budgeting should move towards a process that locks in funding for on-going projects, before allocating to new projects. Lastly, budgeting of capital and recurrent expenditures should be more closely integrated. More specifically, the authorities should:

- Develop principles/criteria for selecting new projects in annual investment plans, making them specific, operational and policy-oriented.

- Introduce a system of forward baseline estimates for on-going public investment projects ${ }^{87}$, by which decision-makers have a full picture (by year) of the forward funding requirements for the efficient implementation of the portfolio of on-going projects (and of the most important projects within them) before they make decisions concerning new projects, for which forward estimates should also be available.

- Design and introduce a credible rolling, medium-term budget framework, which is attuned to the needs of PIM. When rolling over the medium-term budgetary perspective, use the second and third years of the previous year's framework as starting point for updating and extending expenditure plans.

87 Forward baseline estimates should also be prepared for recurrent expenditures as well. These are estimates of the expenditures required if policies remain unchanged, i.e., with not new spending initiatives. Forward baseline estimates for both capital and recurrent expenditure are essential components of a medium-term budgeting systems. 
- Push ahead with developing and implementing a strategy for cleaning up the portfolios of ongoing projects so as to reduce them to a level such that all retained projects can be efficiently implemented and there is some fiscal space for high priority new projects. The clean-up should involve finalising stalled or very slow moving projects (those which have been 'drip-funded), or closing them down for good. This should involve reassessing those stalled projects that are still regarded as priorities after an initial screening, in the same way as new projects to confirm social profitability, but on a sunk cost basis.

- Once the portfolio cleanup has been completed, begin prioritizing new projects separately from on-going projects, after the capital baseline has been established and agreed, and the fiscal space remaining for new projects has been determined. Make firm decisions about medium-term allocations of funding for on-going projects before allocating any remaining funding for new projects. This is best done in the framework of the MTEF/three-year stage budget-finance plan

- Once a more rigorous appraisal process is firmly established, introduce a formal gatekeeping function, whereby projects included in budget requests are reviewed centrally to ensure that they have been properly appraised, have obtained the necessary approvals and are directly aligned with the priorities of their respective sectors.

- Require budget requests for investment projects to include projections of operating and maintenance expenditures for the new facilities once commissioned. 



\section{Project Implementation}

\section{What Does Good Practice Look Like?}

160. There are five main components of good practice in project implementation:

- Guidance on implementation: Comprehensive guidelines on project implementation and management should exist.

- Implementation plans and clear accountability: There should be clear organizational and management responsibilities and accountabilities for delivering on a detailed project implementation plan.

- Procurement arrangements that incentivize achieving value for money with integrity through contracting: Procurement should be efficient, competitive and transparent, and there should be fair recourse through an effective mechanism for raising complaints, which should ideally be independent.

- Timeliness and predictability of in-year funding:There should be predictability in the availability of funds for commitment and payment of project expenditures, and funds should be released in a timely and efficient way over the year so as not to impede efficient implementation.

- Regular and appropriately detailed reporting on implementation: A responsive monitoring system (see Section 10, Project Adjustment, below) requires sufficiently regular and detailed reporting, according to the focus of the monitoring - project-level or portfolio-level. For multiyear projects, financial reporting should be against total project costs and not just annual allocations.

161. Effective management arrangements are necessary to make sure that capital investment projects are delivered on time, to budget and to specification, once a firm funding decision has been made. Good guidance on appropriate arrangements for implementing projects is important in making management systems more effective. This can either be issued centrally or, as is more usually the case, at the sector-level by line ministries or subordinated agencies. Some of the required information could be available elsewhere, in procurement or accounting legislation and regulations for example, but in the better PIM systems these would be referenced in a single document that is continually updated. Ideally, basic guidance should cover:

- The roles of project managers and supervisors;

- Delegations of authority for certifying works and making decisions for deviations from plan;

- Overview of government procurement policies and practices;

- Financial management and reporting requirements;

- Monitoring of implementation progress;

- Procedures for making project adjustments; and

- Arrangements for project completion and post-completion reporting.

In terms of the form of the arrangements set out in the guidance, management is made more effective by having a clear division of accountability and with each individual unit acting within the confines of this accountability. 
162. Projects need to have comprehensive implementation plans and a clear allocation of responsibilities for delivering on them. Plans should incorporate a timeline, including critical path (for major projects) and key milestones, realistic and risk-based fit-for-purpose procurement plans and an expenditure cash flow forecast. Systems need to be in place to monitor and manage total project costs - not just annual allocations - and individual contracts within larger projects.

163. A sound public procurement system is vital for public investment management because capital projects are generally implemented on behalf of government by contractors. Such a system needs a well-defined legal framework that embodies transparency and competition as the means for obtaining fair and reasonable prices, and overall value for money. It also requires a system that allows the submission and resolution of complaints in a fair, open, independent and timely manner. Public dissemination of information on procurement processes and outcomes is an important element of transparency. Procurement processes will naturally match the complexity of the investments; however, in cases of complex investments, special attention much be paid to ensure that this does not add any undue delays to the investment cycle, by ensuring that adequate actions are undertaken by all stakeholders to reduce any potential delays, specifically in relation to the clearance processes. Once signed, contracts need to be managed in a professional and disciplined way.

164. Efficient project implementation requires that the funds approved in the budget be made available in year to the responsible budget entity in a predictable and timely fashion. An unpredictable flow of funds makes it difficult to enter into and honour commitments. Overly complex and detailed budget execution controls could also slow down implementation by delaying funds releases and the necessary authority to commit or spend funds. Slowing down implementation puts back the time when the benefits of the project start flowing. Severe cash shortages leading to a stopgo implementation can increase total project costs because of remobilisation charges.

165. Reporting needs to be calibrated to the needs of monitoring at different levels in the system. Project managers need more detailed and more regular reports than those monitoring the portfolio as a whole. Day-to-day monitoring - and the associated reports - are vital for efficient implementation; at the same time, less detailed and less regular, but more analytical reports, need to arrive at higher levels in the system. This is so that portfolio managers can take a view on progress in implementing the investment program as a whole and identify at-risk projects requiring attention from senior decision-makers and further follow-up. 


\section{Assessment of Project Implementation in Vietnam}

166. The sample survey of projects produced some useful information on project implementation, which is summarised in Table 10 and 11 for transport and education project respectively.

\section{Table 10: Summary Findings on Project Management and Implementation Planning for Transport Projects in the Project Sample}

\begin{tabular}{|c|c|c|c|c|c|c|}
\hline \multirow[b]{2}{*}{ Project } & \multirow[b]{2}{*}{$\begin{array}{l}\text { Main } \\
\text { Funding } \\
\text { Source }\end{array}$} & \multicolumn{3}{|c|}{ Project Management } & \multicolumn{2}{|c|}{ Implementation Planning } \\
\hline & & $\begin{array}{l}\text { Q35: Adherence to } \\
\text { formal guidelines } \\
\text { on project } \\
\text { management }\end{array}$ & $\begin{array}{l}\text { Q37: Hierarchy } \\
\text { of project } \\
\text { management } \\
\text { responsibilities }\end{array}$ & $\begin{array}{l}\text { Q38: Project } \\
\text { Steering } \\
\text { Committee }\end{array}$ & $\begin{array}{l}\text { Q39: Detailed } \\
\text { implementation } \\
\text { plan }\end{array}$ & $\begin{array}{c}\text { Q40: } \\
\text { Procurement } \\
\text { plan }\end{array}$ \\
\hline $\begin{array}{l}\text { 1. Hung } \mathrm{Ha} \\
\text { Bridge }\end{array}$ & ODA & (Yes) & $\begin{array}{l}\text { Yes - project } \\
\text { owner (MOT) } \\
\text { assigns PMU } \\
\text { to manage } \\
\text { implementation }\end{array}$ & (Yes) & $\begin{array}{l}\text { Yes - but plan } \\
\text { was not realistic }\end{array}$ & Yes \\
\hline $\begin{array}{l}\text { 2. Hanoi-HCMC } \\
\text { Railway } \\
\text { Bridges }\end{array}$ & ODA & (Yes) & Yes - as above & (Yes) & $\begin{array}{l}\text { Yes - but plan } \\
\text { was not realistic }\end{array}$ & Yes \\
\hline $\begin{array}{l}\text { 3. Da Nang- } \\
\text { Quang Ngai } \\
\text { Expressway }\end{array}$ & ODA & $\begin{array}{l}\text { Yes - project } \\
\text { operations manual } \\
\text { approved and } \\
\text { applied }\end{array}$ & Yes - as above & (Yes) & Yes & Yes \\
\hline $\begin{array}{l}\text { 4. Terminal } \\
2 \text { Noi Bai } \\
\text { Airport }\end{array}$ & ODA & Yes & Yes - as above & $\begin{array}{l}\text { Yes - } \\
\text { Government } \\
\text { Committee } \\
\text { for Major } \\
\text { Transport } \\
\text { Projects \& } \\
\text { Ministry } \\
\text { Committee } \\
\text { for T2 }\end{array}$ & Yes & Yes \\
\hline $\begin{array}{l}\text { 3. Nghi Son - } \\
\text { HCMC Road }\end{array}$ & $\begin{array}{l}\text { State } \\
\text { budget }\end{array}$ & No & Yes - as above & (Yes) & Yes & Yes \\
\hline $\begin{array}{l}\text { 4. Moi Market- } \\
\text { Chu Market } \\
\text { Road }\end{array}$ & Bond & No & Yes - as above & (Yes) & Yes & Yes \\
\hline $\begin{array}{l}\text { 5. Mekong } \\
\text { Delta } \\
\text { Transport } \\
\text { Infrastructure }\end{array}$ & ODA & (Yes) & Yes - as above & $\begin{array}{l}\text { Yes - chaired } \\
\text { by Vice- } \\
\text { Minister for } \\
\text { Transport }\end{array}$ & $\begin{array}{l}\text { Yes - but plan } \\
\text { was unrealistic } \\
\text { (poor FS) }\end{array}$ & Yes \\
\hline $\begin{array}{l}\text { 6. National } \\
\text { Highway No. } \\
1 \text { Extension }\end{array}$ & Bond & No & Yes - as above & (Yes) & $\begin{array}{l}\text { Yes - but realism } \\
\text { depends on } \\
\text { timely site } \\
\text { handover }\end{array}$ & Yes \\
\hline $\begin{array}{l}\text { 7. Hau River } \\
\text { Navigation } \\
\text { Channels }\end{array}$ & Bond & $\begin{array}{l}\text { No - Decree No } \\
12 / 2009 / N D-C P \\
\text { on management } \\
\text { of construction } \\
\text { projects }\end{array}$ & Yes - as above & (Yes) & $\begin{array}{l}\text { Yes - but plan } \\
\text { was unrealistic }\end{array}$ & Yes \\
\hline $\begin{array}{l}\text { 8. Bim Son } \\
\text { Bridge }\end{array}$ & Bond & No & Yes - as above & (Yes) & Yes & Yes \\
\hline
\end{tabular}

* Initially answered 'Yes' by respondents but, on closer examination of detailed responses, it was found that the question has not been properly understood. Detailed responses only made reference to elements of the legal and regulatory framework relating to implementation issues, but not to specific guidance on project management. 
Table 11: Summary Findings on Project Management and Implementation Planning for Education Projects in the Project Sample

\begin{tabular}{|c|c|c|c|c|c|c|}
\hline \multirow[b]{2}{*}{ Project } & \multirow[b]{2}{*}{$\begin{array}{l}\text { Main } \\
\text { Funding } \\
\text { Source }\end{array}$} & \multicolumn{3}{|c|}{ Project Management } & \multicolumn{2}{|c|}{ Implementation Planning } \\
\hline & & $\begin{array}{c}\text { Q35: } \\
\text { Adherence } \\
\text { to formal } \\
\text { guidelines } \\
\text { on project } \\
\text { management }\end{array}$ & $\begin{array}{l}\text { Q37: Hierarchy } \\
\text { of project } \\
\text { management } \\
\text { responsibilities }\end{array}$ & $\begin{array}{l}\text { Q38: Project } \\
\text { Steering } \\
\text { Committee }\end{array}$ & $\begin{array}{c}\text { Q39: Detailed } \\
\text { implementation } \\
\text { plan }\end{array}$ & $\begin{array}{c}\text { Q40: } \\
\text { Procurement } \\
\text { plan }\end{array}$ \\
\hline $\begin{array}{l}\text { 1. Training Centre } \\
\text { - National } \\
\text { Economics } \\
\text { University }\end{array}$ & $\begin{array}{l}\text { State } \\
\text { Budget + } \\
\text { Own }\end{array}$ & $\mathrm{No}^{*}$ & Yes & (Yes) & No & No \\
\hline $\begin{array}{l}\text { 2. Lecture Hall } \\
\text { - Nat. Inst. } \\
\text { of Education } \\
\text { Management }\end{array}$ & $\begin{array}{l}\text { State } \\
\text { Budget }+ \\
\text { Own }\end{array}$ & No & No response & No response & No response & No response \\
\hline $\begin{array}{l}\text { 3. Viet Duc } \\
\text { University }\end{array}$ & ODA & No response & Yes & $\begin{array}{l}\text { Yes, but } \\
\text { dissolved }\end{array}$ & Yes & Yes \\
\hline $\begin{array}{l}\text { 4. Lecture halls } \\
\text { - HCMC Uni. } \\
\text { of Tech. \& } \\
\text { Education }\end{array}$ & $\begin{array}{l}\text { State } \\
\text { Budget + } \\
\text { Own }\end{array}$ & No response & Yes & $\begin{array}{l}\text { (Yes) - } \\
\text { 'management } \\
\text { unit of project } \\
\text { documentation' }\end{array}$ & Yes & Yes \\
\hline $\begin{array}{l}\text { 5. Lecture halls - } \\
\text { HCMC Uni. of } \\
\text { Law }\end{array}$ & $\begin{array}{l}\text { Own + } \\
\text { State } \\
\text { Budget }\end{array}$ & $\mathrm{No}^{*}$ & Yes & Yes - no details & Yes & Yes \\
\hline $\begin{array}{l}\text { 6. Kien Giang } \\
\text { campus - } \\
\text { Nha Trang } \\
\text { University }\end{array}$ & $\begin{array}{l}\text { State } \\
\text { Budget + } \\
\text { Own }\end{array}$ & $\mathrm{No}^{*}$ & Yes & (Yes) - 'PMU' & Yes & Yes \\
\hline $\begin{array}{l}\text { 7. Long Binh } \\
\text { Campus - } \\
\text { HCMC Open } \\
\text { University }\end{array}$ & & & & & & \\
\hline
\end{tabular}

* Initially answered 'Yes' by respondents but, on closer examination of detailed responses, it was found that the question has not been properly understood. Detailed responses only made reference to elements of the legal and regulatory framework relating to implementation issues, but not to specific guidance on project management.

\section{Project management arrangements in Vietnam are generally sufficiently robust, but there is scope for further strengthening and addressing weaknesses in application, particularly at the province level.}

167. Vietnam lacks national or sector-specific guidance on project management for fully domestically funded projects. It is clear from the detailed responses to the question in the sample survey on project management guidelines that it had not been fully understood, probably because there is limited appreciation of what such guidance might look like. The detailed responses listed many different legal and regulatory instruments most of which had little relevance to the specific issue of project management. None of the detailed responses mentioned specific guidance on project management. The instrument with the most relevance to the issue seems to be Decree No.12/2009/ $\mathrm{ND}-\mathrm{CP}^{88}$, but this was only mentioned by one respondent. In the cases of ODA projects, it would be usual for most donors and IFIs to issue their own guidance on project management, although this was

88 This decree has subsequently been replaced by Decree No. 59 regulating implementation of the Construction Law. 
not mentioned in the responses, except indirectly in relation to Da Nang-Quang Ngai Expressway. There appears to be an acute need for clear and concise guidance on both project and contract management, differentiating clearly between the two. These two aspects are often mistaken for one another. Only in cases where a strategic approach to project management is adopted, can contracts be more proportional and appropriate for the corresponding projects, hence enabling effective contract management.

168. International experience is that project management arrangements set out in PIM-related laws and regulations need to be supplemented by more detailed instructions. These detailed instructions are usually issued in the form of a project management manual, which can either be generic, sector-specific or agency-specific. For example, the US Federal Transit Administration (USFTA) issues a 'Construction Project Management Handbook'89 for urban mass transit projects (which is a substantial document of 166 pages). Individual agencies may then prepare their own project management guidance on the basis of the general or sector-specific guidance. In line with the previous example, Box 17 summarizes the contents of the project management manual for one particular mass transit operation, the Metrolink commuter rail system serving Southern California. Note that such guidance usually covers the whole project cycle, including upstream stages, but from the management perspective, as opposed to the methodological perspective covered in the project appraisal manuals mentioned in Section 5. 


\section{Box 17: Metrolink Project Management Manual - Table of Contents}

\section{Introduction}

1.1.Purpose and structure of the manual

1.2.Metrolink's service territory

1.3.Typical Metrolink projects

1.4.Safe working

1.5.Non-construction projects

2. Metrolink Project Structure

2.1.General

2.2.Strategy and Capital Planning Group

2.3.Project Executive Group

2.4.Project Sponsor

2.5.Program Manager

2.6.Project Manager

\section{The Project Life Cycle}
3.1.Strategic Phase
3.2.Initiation Phase
3.3.Planning Phase
3.4.Design Phase
3.5.Bid Award Phase
3.6. Construction Phase
3.7. Closeout Phase

\section{Procurement/Contract Delivery}

4.1.Teaming with Technical Groups

4.2. Contract Management

4.3.Coordination with the Program Management Office

\section{Project Manager Role and Responsibilities}

5.1. Qualifications and Training

5.2. How Project Managers Are Assigned

5.3.Project Manager Work Loads

5.4.Project Manager Duties

\section{Program Management Office}

Source: Metrolink Project Management Manual, 2011.

169. It is good practice for the project manager to prepare a project operational manual for an individual project, setting out how an individual project will be managed, executed, monitored, controlled and closed. This would be based on the higher level guidance (whether generic, sectorspecific or agency-specific), and should set out the project delivery strategy, organization and management structure, assignment of responsibilities between the project owner/sponsor and contractors, and the delegation of management and financial authority within the project team. This is common practice for ODA projects, an example being the World Bank's project operational manual, which is mentioned in relation to the Da Nang-Quang Ngai Expressway project in Table 9. This manual outlines the operational guidelines and procedures to be followed in implementing the Bank project, including the processes of identifying, preparing, selecting, procuring, managing and monitoring the implementation of sub-projects, technical assistance and all other related project activities. It is expected to be used by staff of the project management unit, municipalities, contractors, engineers, 
environmental and social assessment consultants, and other relevant bodies implementing the project. All supporting documents, forms, and templates required for the implementation of the components and for management activities are included in annexes to manual and form an integral part of it. The project operational manual sets out the guidelines for: i) the financial management of allocated resources; ii) the technical management, monitoring and evaluation of the components activities; and iii) the procurement and contracting procedures for required goods, works and services.

170. Designated project management units (PMUs) are responsible for project management in Vietnam. There have been important changes recently to the way PMUs are set up and operate (see below), but most of the projects in the project sample were started using the previous approach which existed before the Construction Law was updated. All of the projects in the sample are implemented using the "old" PMU system. PMUs are assigned the role by the project owner and are responsible, through their management structure, for delivering a project on time, to budget and to specification. They have their own organisational structure and professional staff, adjusted for project size. PMUs are established outside the organisational structure of the project owner. They can be permanent, responsible for all projects in a sector and geographic location as they arise, or temporary and project specific. In the project sample, the largest projects tend to have dedicated PMUs. PMUs are largely country-owned organisational structures and not simply in place to manage implementation of ODA projects, although they do this too.

171. The Construction Law 2014, together with its supporting regulations, updates the legal and regulatory framework for PMUs for construction projects. In the past, questions were raised about the excessive number of PMUs (and the associated quality of human resources, because of limitations on remuneration of staff ${ }^{\circ 0}$ ). The $\mathrm{CL}$ seeks to overcome some of the problems of the previous system by reducing the number of PMUs and making them more professional. It allows for the creation of specialised project management bureaux at national and regional level. A project management bureau can be designated as the responsible project owner, by the agency promoting the project. The status of these new bureaux had been enhanced, including by making the head of a provincial PMU, for example, equal in rank to the director general of a provincial department and reporting directly to the People's Committee. The professionalization of project management is to be welcomed, but the potential loss of high-level decision-making authority by the agency promoting the project - through the contracting out of the project owner's role - is of some concern.

172. Under a general director and deputy general director, large PMUs are organised in several divisions, each performing different project management functions. The organisational structure of the larger PMUs follows a similar pattern:

- Project management division - monitors progress and quality of works, and accepts the works on completion.

- Economic and planning division - prepares monthly/quarterly/annual progress reports; plans resources; prepares project budget.

- Accounting and finance division - arranges payments and manages other financial matters, including preparation of financial statements.

- Technical engineering division - technical specification and construction quality.

90 'Public Investment Project Management - Lessons from the 2006-2010 Portfolio', World Bank, 2011. This was particularly the case in the under-developed provinces. 
The Deputy Director General of a PMU directs and administers the project(s) and is effectively the project manager, although this title is not used.

173. For high-level decision-making, a senior manager is designated from within the organisation of the managing agency and, while it is not done on a systematic basis, project steering committees ${ }^{91}$ are also ometimes set up to support this manager. When a line ministry is the managing agency, a vice minister is usually appointed as the senior decision-maker for implementation, with ultimate accountability for project delivery. Two transport projects from the sample (both ODA-funded) had proper Project Steering Committee chaired by a Vice Minister ${ }^{92}$. It has apparently been more common practice in the past to establish steering committees, but the current policy is not well-defined. There is potential for putting steering committees on a firmer footing, but if this is done they will need to be properly managed so as to avoid creating bottlenecks.

174. Although detailed project implementation plans and procurement plans are drawn up before implementation begins, their realism depends on the quality of feasibility studies, which is not necessarily dependable. Several projects in the sample indicated that when it came to execution, the implementation plans had to be revised because of feasibility study failings. Although this was not stated, the same would presumably have been true for procurement plans. A perennial problem for Vietnam has been over-optimism concerning the time required for land acquisition (and its cost), which has undermined the realism of implementation plans. There are hints in the response to the survey questions for the project sample that this is still an issue. This suggests the need for designating a formal land acquisition stage, which must be completed before works can be budgeted and commenced. Verification of readiness-to-go should then be a precondition for project implementation to start (and for budgeting of construction works). The authorities have been considering similar arrangements, but these have proved difficult to put into practice, because of difficulties in separating funding of land acquisition from funding of works ${ }^{93}$. In South Africa, land acquisition is part of the project planning and design phase, which forms a separate part of the project budget and precedes other procurement processes. This arrangement recognises that land acquisition is normally a long process that is best completed before commencement of the execution phase of the project. Box 18 indicates the steps in land acquisition in South Africa and the approximate time allowed for each.

91 Also known as project boards.

92 The distinction between a PMU and a steering committee was not well understood and, while many of the answers were in the affirmative concerning the steering committee, a PMU was usually being referred to.

93 Allocating funding for land, when there is no approved funding for the project, has turned out to be a sticking point. The idea of a fund for land acquisition has been cleared, but there remain problems establishing procedures for allocating the fund to projects in an effective and transparent way. 


\section{Box 18: South Africa's Land Aquisition Process}

1. Project registration (3-6 months)

2. Design topographical survey (8-12 weeks)

3. Cadastral key plan (2-8 weeks)

4. Preliminary engineering design (1-4 months)

5. Property report (1-4 weeks)

6. Final engineering design (1-2 months)

7. Land acquisition key plan (1-2 weeks)

8. Property data sourcing (1-2 months)

9. Acquisition plan (2-3 weeks)

10. Acquisition documentation (2-5 weeks)

11. Acquisition negotiations (4-8 weeks)

12. Sub-division of land (6-24 months)

13. Conveyancing (3-5 months)

Source: Authors' discussions with South African infrastructure planner.

175. Discussions with key actors, indicated that the PIL is already having an effect on the quality of planning and the deliverability of projects. The more rigorous approach to preparing and verifying projects (even if there are still some weaknesses at certain stages), combined with more structured verification and decision-making is beginning to show in increasingly realistic implementation plans and fewer delays.

Vietnam does not currently have a fully functioning PIM information system for centralised collection and management of information on implementation progress - as a basis for active monitoring - although important efforts are being made to establish such a system.

176. There is regular reporting on project implementation. Reporting requirements - contents, frequency and timeliness - are established in the Construction Law ${ }^{94}$. For projects in the sample, PMUs report to project owners on a monthly, quarterly and annual basis, on both financial and physical performance. Reports indicate variances from plans and analyse causes. The flow of information to the planning agencies (MPI and DPIs) ${ }^{95}$ is less frequent - twice yearly - less reliable and in cumbersome formats. This makes it difficult for the planning agencies to assemble a comprehensive picture of portfolio performance and identify projects at risk of delivery failure.

177. The MPI is in the process of establishing a centralised PIM information system for electronic reporting on projects funded from the state budget, including ODA. Such a system would be an important step forward in PIM system development. The roll-out of the system was initiated at the end of 2016 through a circular from $\mathrm{MP}^{96}$, and it is now capturing reporting on around 9,000 projects. The reporting regime has recently been further strengthened through the issuance of another circular ${ }^{97}$ with wide applicability. This governs reporting on the preparation, monitoring, and evaluation of implementation of public investment plans, with a focus on information related to the medium-

94 Articles of 68 and 69 of the Construction Law.

95 Reports are submitted to DPIs, which in turn report to MPI on province projects

96 Circular No.13/2016/TT-BKHDT of 29/09/2016

97 Circular No.3/2017/TT-BKHDT of 25/04/2017 
term and annual investment planning process. The circular requires use of the electronic system for filing reports on implementation progress, but also on other aspects of the project cycle linked to formulation and upstream decision-making. It does not require project owners to generate additional information, merely to share more of the existing information with the centre, using the electronic system.

178. Linking the MPI system to TABMIS ${ }^{98}$, under MOF, will be essential, as will close involvement of the MOF in its development. Ensuring accurate and timely information on financial execution of projects is important for the usefulness and credibility of the PIM information system. Linking to TABMIS data is rightly given priority as part of the MPI's on-going PIM reform agenda. Technically the information exchange seems feasible, but the organisational dimensions are more challenging, since the systems belong to different ministries and information sharing is never straightforward anywhere, but particularly in Vietnam. High-level agreement will be required between the most senior decisionmakers in each ministry at the earliest opportunity, followed by the establishment of an inter-ministry working group with a tight deadline for resolving the constraints - one of which will be legitimate concern about preserving the integrity of TABMIS for security reasons - and completing integration at the earliest opportunity.

179. The MOF's on-going work to develop a data warehouse, which regularly downloads TABMIS data and makes it available, could be one solution to the exchange of financial information, but has drawbacks in terms of the timescale involved. The data warehouse concept, which bears a resemblance to the Colombian case mentioned below, is expected to be completed by 2019. If this is the chosen route to improving financial execution data in the PIM information system, then close coordination between the MPI and MOF during its development will be important, so as to ensure requirements are met. The drawback of this approach would be the long time to completion ${ }^{99}$, which would handicap improvement of the MPI's information system for the next two years. An earlier direct link to TABMIS therefore seems preferable and would be consistent with the conception of TABMIS as an integrated financial management tool for budget managers ${ }^{100}$. Whatever the chosen solution, collaboration between the MPI and MOF will be essential, both during development and then during operation.

180. The information system's capability to capture and report non-financial information on projects needs to be expanded. As well as resolving the issue of information on financial execution, the MPI's PIM information system will also need further development so that it can better capture nonfinancial performance indicators, and produce, at the press of a button, the kind of reports that will be of use to public investment supervisory bodies and to the Government. In the longer term, public access to summary information on portfolio and project performance - a feature of the Colombian case discussed below - should be explored.

181. Robust information systems form the backbone of some of the more advanced international experience in PIM. Good information and information sharing, including with the public, are critical for enhanced PIM system performance. Advanced information systems are a particular feature of PIM in some South American countries, notably Chile and Colombia. Colombia now has a unified information system built up from different components, which serves wider PFM and development planning purposes, beyond PIM. The unified system reduces duplication of data

98 TABMIS is Vietnam's integrated financial management information system used for budget execution, reporting and accounting.

99 This is necessary because of the wide range of needs the system is expected to serve.

100 It would also be consistent with the usual functions of an integrated financial management system. 
entry, improves accuracy and allows data sharing across government. The design of the unified system is shown in Box 19. The key things to note are:

- The database of projects - the BIPM - which captures projects from inception (not just when they are budgeted) so that all key upstream processes and decisions are captured, as well as the rest of the project cycle;

- The integration with the budget execution, reporting and accounting system, SIIF (the equivalent of TABMIS);

- The module for monitoring both physical and financial implementation (SPI); and

- Linkage to the system for monitoring and evaluation of the wider national development plan (NDP).

Interoperability of information systems should also be Vietnam's longer term aim. As an example, a significant effort is currently being made to collect accurate data for procurement through the e-GP (Electronic Government Procurement) system, which should eventually tie in with the PIM system.

\section{Box 19: Columbia's Unified System for Public Finance and Investment (SUIFP)}

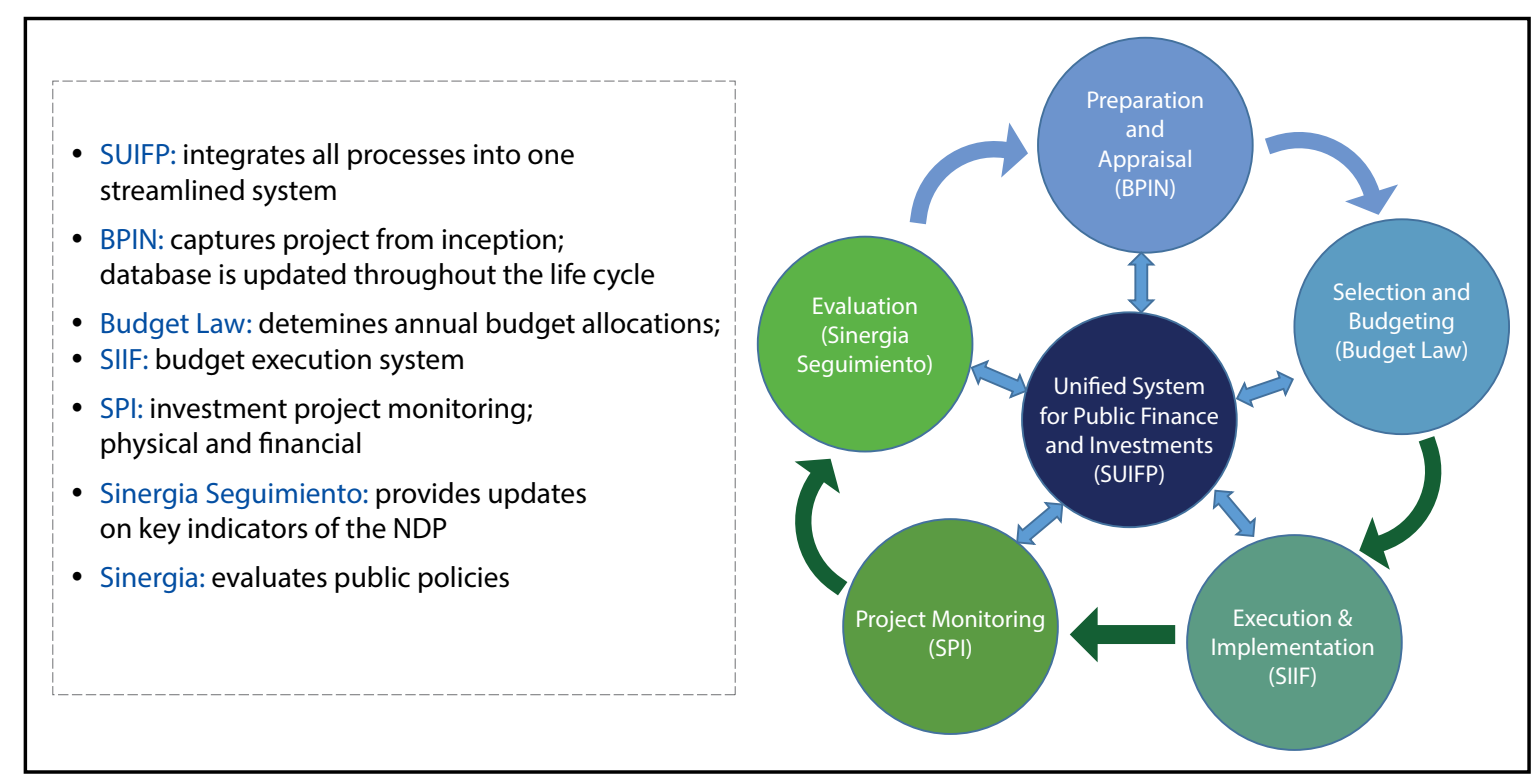

Source: 'Sistema Unificado de Inversiones y Finanzas Públicas' a presentation by the National Department of Planning, Colombia, 2015. 


\section{Summary Assessment of Project Implementation}

\begin{tabular}{l|}
\hline Key Functionality \\
\hline $\begin{array}{l}\text { Projects are } \\
\text { scrutinised for } \\
\text { implementation } \\
\text { realism and then } \\
\text { implemented with } \\
\text { regard to efficiency. }\end{array}$
\end{tabular}

2. Implementation plans, clear accountability for delivery and checks on readiness to go.

\section{Current Arrangements in Vietnam}

At the higher level, project implementation is reasonably well regulated in Vietnam, through a specific laws (the PIL and the Construction Law), and supporting government and ministerial decrees and notices. One weakness seems to be a shortage of specific operational guidance on managing project implementation, either at the national level or (probably more useful) sector level.

Detailed implementation plans are prepared before implementation begins, but often suffer from a lack of realism, frequently as a result of poor quality feasibility studies.

There is anecdotal evidence that the situation has improved following introduction of the PIL, which has helped improve the quality of planning and the timely execution of projects. Simultaneous planning/design and implementation is no longer allowed, removing a problem that had sometimes beset execution.

Delay in site acquisition is a perennial problem in Vietnam is, often holding up planned implementation.

Project managing agencies assign professional project management units (PMUs) to manage implementation on their behalves. PMUs are accountable for delivering projects on time, to budget and to specification.

For the most significant projects, a project steering committee under a deputy minister (where a line ministry is the managing agency), or the equivalent position at province level, is sometimes created, but this is not a consistent policy. Otherwise a nominated deputy minister (or equivalent at province level) is responsible for providing high-level direction to a project - 'the senior responsible official' - from within the organisation of the managing agency.

\section{Procurement} arrangements that incentivise value for money in contracting.
$\mathrm{Pl}-19$ - transparency, competition, and complaints mechanisms in procurement - was rated C+ in the 2013 PEFA assessment. The worst rating, $D$, related to the use of competitive procurement methods. The assessment found that, although the law makes this the default method of procurement, there are a number of derogations from its application, which are frequently used in practice. The result is that open competition was found to account for less than $60 \%$ of the total procurement by value. As an indicator of the scope for improvement, international financial institutions are not prepared to use country procurement systems for the projects that they fund.

The ratings were rather higher, $B$, in relation to the public availability of information and an independent complaints mechanism. 


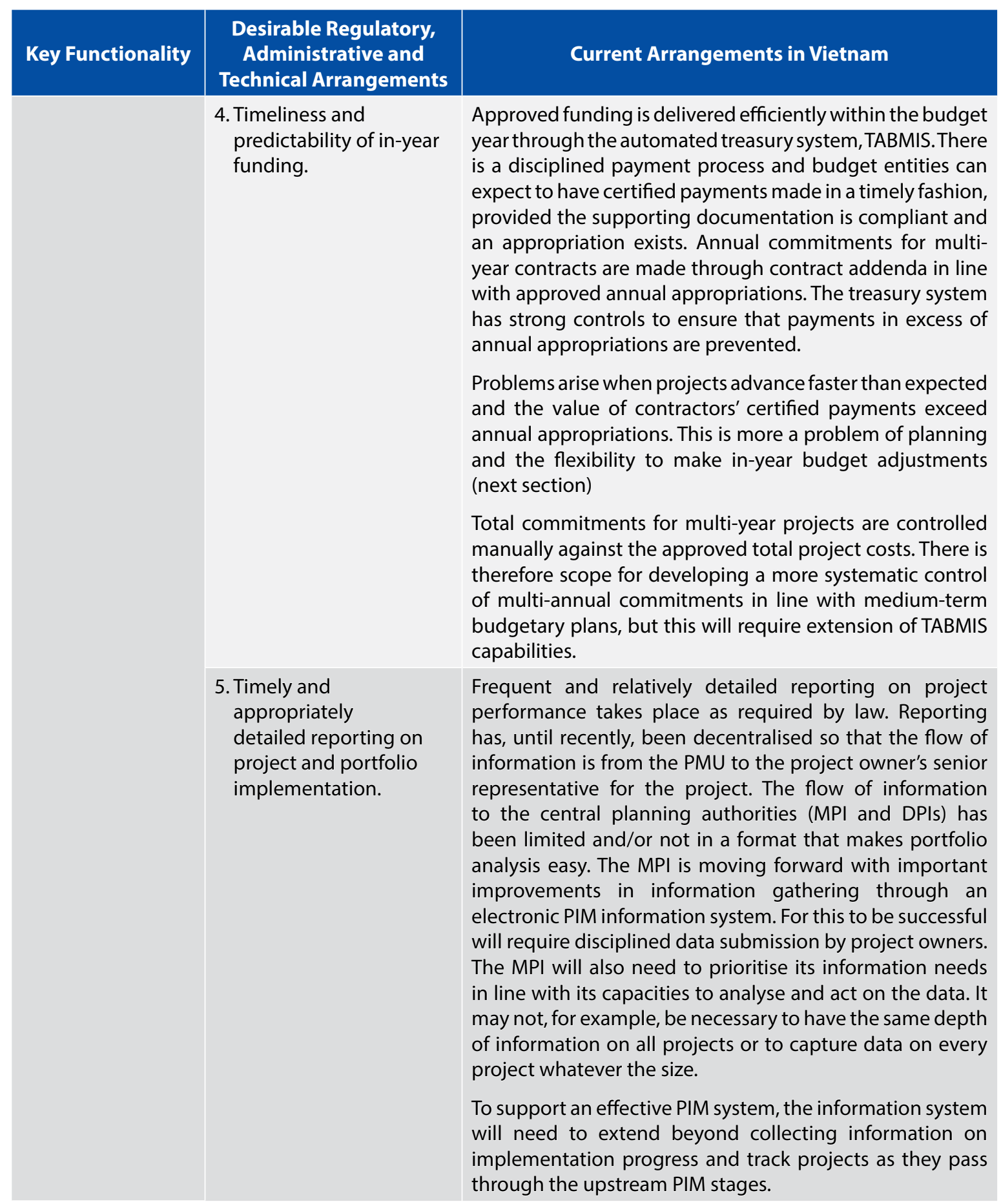




\section{Recommendations}

182. Broadly speaking, there is room for further strengthening of project management arrangements. In addition, greater use of competitive procurement methods has the potential to improve value for money. The more detailed recommendations are for the authorities to:

- Develop detailed instructions on managing project implementation and require a project implementation management plan for all major projects.

- Review the consistency and completeness of legislation on PMUs.

- Review the policy on establishing project steering committees (project boards) for major projects on a more systematic basis.

- Proceed with the development of the PIM information system, giving priority to establishing the link to TABMIS, so as to secure accurate information on financial execution of projects, but also enhancing the system for coverage of the full project cycle and for collection of better performance information.

- Explore the possibilities of extending TABMIS capabilities to allow greater control over multiyear contractual commitments. 


\section{Project Adjustment}

\section{What Does Good Practice Look Like?}

\section{There are three features of a good process for managing adjustments to projects during implementation:}

- Responsive monitoring: Implementation progress should be actively followed ${ }^{101}$, resulting in early detection of deviations from plan and their resolution at a level in the hierarchy of decision-making that balances efficiency against oversight and accountability considerations.

- Judicious use of virement: To facilitate high rates of execution, transfers of annual budget allocations between budget headings should be possible, subject to an approval process that balances efficiency against oversight and accountability considerations.

- Fundamental review: There should be a clear mechanism for triggering fundamental reviews of failing projects or those where baseline conditions have changed unfavorably.

184. Responsive monitoring involves putting in place systems to ensure problems and emerging risks are identified in a timely fashion, devising solutions and then following up. Regular, accurate and informative reporting (see Section 7, Project Implementation), is a prerequisite of responsive monitoring, but reporting alone is not very useful if it is not embedded in a reactive system. Monitoring needs to be formalised procedurally so that problems and potential problems are registered, corrective actions are designed and implemented, and their success verified (and further actions taken if unsuccessful). Not all implementation problems need to be escalated to higher levels of decision-making, although these superior levels should be kept informed. In an efficient monitoring system, authority is delegated to the level best equipped to address implementation issues, and only unresolved problems and major problems requiring high-level guidance or decisions are escalated. Not all systems have centralised monitoring of project implementation by planning or finance ministries, but there is an increasing recognition that for major projects this is a prerequisite. At this level in the system, monitoring portfolio aggregates, e.g., portfolio execution rates and share of the portfolio at risk of delivery shortfalls, and resolution of systemic issues are also important, along with project specific issues that cannot be satisfactorily resolved at lower levels.

185. Disciplined budget execution is important, but excessive rigidity can hinder efficient portfolio implementation by preventing transfers from slower to faster moving projects within the budget year. The better performing systems allow budget holders to make smaller reallocations between projects in their portfolios, subject to a designated maximum threshold. More significant reallocations would be referred to the planning or finance ministries for approval, while the most significant transfers would usually require government and legislative approval (usually through an in-year supplementary budget). Reallocations between budget holders would usually require government and legislative approval. Virement rules - including the thresholds for different levels of decision-making over in-year transfers - are usually set out in a country's budget systems law or budget code. It is also usually good practice for the legislation to safeguard capital spending by preventing in-year transfers from capital to recurrent allocations. 
186. Significant adjustments to projects in terms of costs, timetable and specification can, along with changes to demand conditions, affect the social profitability of projects and procedures should be in place for re-assessment of such projects. Re-assessment should be on a sunk cost basis ${ }^{102}$ and the costs of terminating the project also need to be taken into account. If a fundamental review indicates that a project is no longer socially profitable, procedures should exist for initiating a redesign of the project to reduce costs or for terminating the project should no such solution be found. Fundamental reviews are usually triggered when costs (expressed in constant prices) exceed a predetermined threshold or when forecast benefits seem likely to fall short of appraisal estimates. Reviews should not be triggered by minor adjustments, as this could overburden analytical capacities and risks turning the review into an administrative hurdle, rather than an in-depth reassessment of the continued viability of a project. Good reporting and monitoring are evidently essential to ensuring that reviews are triggered when required.

187. Fundamental reviews happen between appraisal and implementation, if factors emerge that change the original basis for the feasibility study, and during implementation. South Korea represents one of the better examples of a systematic process for carrying out reassessments of major projects (see Box 20). This is supported by a system for tracking actual against approved total costs - the Total Project Cost Management System - which provides the information required to trigger a review. Over the years it has resulted in significant cost savings through realigning projects within affordable cost estimates and through cancellation of projects that no longer represented good value for public money.

102 It is important in any re-assessment of feasibility to use costs that are net of sunk costs. Sunk costs are resources that have already been used up in project implementation and can no longer be used for any other purpose, i.e., their opportunity costs is zero. For example, if a bridge project has been partially constructed (in physical terms), the costs associated with the completed works should not be counted in the new economic feasibility study. 


\section{Box 20: Re-assessment and Adjustment in South Korea}

In South Korea both a re-assessment of demand and a reassessment of feasibility can be triggered by significant changes in project conditions that risk undermining the forecast economic returns from the investment:

Re-assessment of Demand Forecast (RDF - introduced in 2006)

Demand forecasts for major projects are re-examined when there are important changes in the project environment that could affect the continued adequacy of the forecasts. The RDF can be carried out during any phase of the project cycle from planning to construction.

Re-assessment Study of Feasibility (RSF - introduced in 1999 and strengthened 2006)

A RSF is performed for projects in the Total Project Cost Management System if:

- The total cost for a project increases by more than 20 percent in real terms (excluding land acquisition) compared to the previously approved cost.

- When the demand forecast for a project falls by 30 percent or more (on the basis of the RDF).

The RFS effectively involves repeating the SCBA carried out for the feasibility study, but with revised cost and/or demand estimates. On the basis of the results of the new RFS, a decision is taken on whether to continue, re-scope or stop the project. Re-scoping involves looking at ways of reducing the size and cost of the project to achieve continued viability and avoid cancellation.

24 out of 140 projects subject to RSF were stopped during the period 2006-2010. Total project cost savings of $18 \%$ were made (compared to requested increase) on projects that continued.

Sources: Presentation by Korean Development Institute to International Conference on Public Investment Management in Hanoi, 2010; 'Public Investment Management Functionality', presentation given in Mongolia by James Brumby, World Bank, 2010; and Guidelines for Total Project Cost Management, Ministry of Strategy and Finance, 2009.

\section{Assessment of Monitoring and Project Adjustment in Vietnam}

Responsive monitoring is taking place at a decentralised level, closer to project implementation, but is not yet well developed at the level of the MPI. The legal framework has improved through the PIL and the Construction Law, and the MPI is making efforts to improve the information base through the recently rolled-out PIM information system. There is, however, further work to be done before the MPI is in a position to be able to identify projects with a high risk of delivery failure and then to ensure that adequate steps are being taken to bring them back on track and, in the worst cases, close them down.

188. The monitoring system set out in Vietnam's legal and regulatory framework for PIM meets good practice specifications. The PIL and the Construction Law provide a firm legal basis for monitoring, with the latter largely deferring to the former ${ }^{103}$. Definitions of terms, responsibilities, scope, methods and procedures for monitoring are detailed in a specific decree on supervision and assessment of investment ${ }^{104}$, which is in conformity with internationally recognised practices for monitoring. Under the broader heading of supervision, the decree makes an important distinction between inspection, which is concerned with legal and regulatory compliance, and monitoring, which

103 As set out in Chapter IV of the PIL on Implementation, Supervision, Inspection and Assessment of Plans for Public Investment and Article 8 of the Construction Law on Monitoring and Evaluation of Construction Investment Projects

104 Decree No. 84/2015/ND-CP. The decree also covers the evaluation stage of PIM - referred to as 'assessment'- in the decree, which is the subject of Section 12 of this report. 
is to do with ensuring 'the project's adherence to its objectives, and schedule, quality, and capacity of determined resources' 105 .

189. A core feature of monitoring, as envisaged, is identifying deviations from plan and developing corrective actions. The decree foresees monitoring of implementation progress in both physical and financial terms, and monitoring of performance once a project is operating. The scope and detail of monitoring varies according to the responsible body - investor/operator, investment decision-maker, regulatory body/public investment authority, and specialised authorities - and their position in the project/PIM hierarchy. Those problems that cannot be resolved at one level in the monitoring hierarchy are to be identified and escalated to the next level up. Ministries and regulatory bodies are required to establish dedicated supervision and assessment ${ }^{106}$ units. At province level, the DPIs are expected to fulfil this role. The frequency of monitoring reports and the timetable for their delivery are also laid out in the decree.

190. There is scope for significantly strengthening MPI's role in centralised monitoring of public investment projects, particularly with respect to problematic or at-risk projects. The MOF also has an important role with respect to financial monitoring and close collaboration between the two organisations is a must (as already indicated in Section 9 with respect to the development and operation of the reporting/information system). The MPI currently has responsibility for establishing the monitoring system and for higher level monitoring of the investment portfolio as a whole, but its role with respect to individual projects is limited. The MPI's broad role in monitoring is established in the $\mathrm{CL}$ and the $\mathrm{PIL}^{107}$ and then specified in more depth in the decree on supervision and assessment. In the latter, it is stated that the MPI is in charge of assisting the Prime Minister in organising supervision (including monitoring) and assessment ${ }^{108}$, and that it is required, among other things, to submit semiannual and annual reports on overall investment supervision ${ }^{109}$ to the Prime Minister. In practice, the $\mathrm{MPI}$ can request line ministries and provinces to submit regular reports on the spending agency's aggregate investment status, or ad hoc reports on major projects (Group A and nationally important projects), but has difficulty in pursuing an active monitoring role with respect to individual projects. The only way it can do the latter is through its irregular inspection function, which is compliance oriented. Regular monitoring is carried out by the investment decision-maker, as it should be, but with limited upward coordination with and information flow to the MPI. The extent to which a central public investment agency gets involved in monitoring individual projects varies from country to country and Vietnam has a choice to make in this respect ${ }^{110}$, but whatever the preference, there is a case for an enhanced role compared to the current one.

105 Article 2.2 of Decree Mo. 84/2015/ND-CP

106 Monitoring and evaluation - M\&E - units.

107 Article 88.9 of the PIL 'Implement, supervise, inspect and evaluate the plans, programs, and projects and other management obligations of public investment.

108 Article 59 of Decree no. 84/2015/ND-CP. The MPI's responsibilities include leading the supervision and assessment of programs and project which investment policies are decided by the Prime Minister, the Government, or the National Assembly, i.e., projects of national importance and Group A projects.

109 Article 2.12 "overall monitoring of investment" means regular and periodic update of information about investment and management of investment by regulatory bodies and local governments; consolidation, analysis, evaluation of information; proposal of mechanisms and policies related to investment management.' 'Overall monitoring of investment' is defined as part of 'overall supervision of investment'.

110 In the UK and New Zealand only major projects are monitored; in Columbia and Chile monitoring of all projects is performed. In both cases, the level of detail is not the same as it is for investors and for investment decision-makers and the focus is on identifying problem projects, i.e., those where deviations from plan are greater than agreed tolerances, and getting them back on track. 
191. Although a supportive legal and regulatory framework is now in place and the technical conditions are improving, Vietnam still has a long way to go before achieving responsive project monitoring in its fullest sense. The PIL and the accompanying regulation have placed monitoring on a firm footing legally procedurally, but operationally there is still a long way to go before the kind of system envisaged is fully functional. Partly it is an enforcement issue. This is made worse by the relative complexity and fragmentation of the system, reflecting the decision-making and accountability arrangements associated with Vietnam's highly decentralised system of government, where a lot of authority is devolved to province level and below. Prior to the PIL there was a heavy emphasis on the inspection part of the supervision function, and the monitoring component, with its emphasis on problem solving and feedback, was not developed to the same extent. Inevitably, it will take time to shift the balance away from an emphasis on verification of compliance and towards enhancing project performance. Establishing effective feedback mechanisms in an administrative culture which is more used to apportioning blame for compliance errors is also something that can only be achieved over a longer time frame.

192. Implementation of the monitoring and evaluation system is hampered by a lack of detailed instructions for the different parties involved. Guidelines on the implementation of the legal and regulatory framework will probably be required to be developed, possibly in the form of a monitoring and evaluation manual, together with building of capacity to support implementation. Although it is heading in the right direction, more work on the development and testing of the PIM information system, together with improvements in the quality and timelines of data submission, will also be required, as discussed in the previous section.

193. The legal provisions are quite strict - probably too strict - when it comes to the need for a fundamental review of a project faced with significant cost increases. Both the PIL, the Construction Law and the supporting regulations ${ }^{111}$ contain provisions covering procedures for adjusting projects. These require re-assessment of projects according to the provisions of the legal and regulatory framework whenever costs change ${ }^{112}$. This seems to indicate a strong requirement for fundamental review, which if interpreted strictly, would require a re-assessment of the case for a project even for minor adjustments. This seems too onerous and inflexible. It would be better to specify thresholds within which adjustments could take place without significant re-assessment work and above which fundamental review would be required. It is also notable that there is no requirement for fundamental review when there is a significant reduction in anticipated benefits - as is the case in good practice countries - although benefits should be reviewed if costs increase. Notwithstanding the above discussion, while appraisal methods remain weak in Vietnam (see Section 6) fundamental review cannot have the same significance it has in stronger PIM systems.

194. The procedures for adjustments of ODA projects are even more cumbersome, and require relevant agencies to review the proposed changes twice. In the first review, the investment managing agency has to send the package with proposed changes to the MPI, which shares with relevant agencies, including the MOF. The MPI then consolidates all the comments and the Prime Minister decides on the changed investment intention. The second review involves the revision of

111 In the UK and New Zealand only major projects are monitored; in Columbia and Chile monitoring of all projects is performed. In both cases, the level of detail is not the same as it is for investors and for investment decision-makers and the focus is on identifying problem projects, i.e., those where deviations from plan are greater than agreed tolerances, and getting them back on track.

112 Article 61.5 of the Construction Law states that: 'The adjustment of investment construction projects shall be appraised and approved.' Article 46.3 of the PIL states that: 'Competent authorities are only entitled to adjust investment program and project after examination and assessment according to regulations set out in this Law.' 
the financing agreement. This process is coordinated by the SBV (for World Bank, ADB, and IMF) or the MOF (for other DPs), and again, comments from the same relevant agencies have to be provided to the coordinating agency.

195. The circumstances and procedures for cancelling poorly performing project are not clear enough. The PIL only allows cancellation of projects by investment deciders ${ }^{113}$, but gives no further details on the circumstances or process for cancellation. If a project is shown to be no longer viable in the face of a significant upward cost adjustment and after taking account of cancellation costs, then it needs to be closed down. Leaving this to the discretion of the investor seems too weak an arrangement, and there needs to be a more clearly defined role for public investment regulatory agencies and investment decision-makers. As an example of practice elsewhere, the position with respect to project adjustment and cancellation in Ireland is summarised in Box 21.

\section{Box 21: Ireland's Guidelines on Project Adjustment}

\section{'Adverse Developments or Changes in Circumstances}

Regular management reports should be prepared by the Sponsoring Agency covering all significant developments relating to the project and its costs. If adverse developments occur, including unforeseen cost increases, which call into question the desirability or viability of the project, the Sponsoring Agency should submit a report at the earliest possible moment to the Sanctioning Authority, detailing the necessary measures proposed to rectify the situation.

Where, despite these measures, increased costs above those already approved are likely to arise, the approval of the Sanctioning Authority for the extra expenditure should be obtained before any commitment is made to accept cost increases. Any application for such approval should outline the reasons for the excess, along with a detailed explanation of why it was not possible to take appropriate measures to offset the increased cost. The viability of the project, given the changed circumstances, should also be reported on.

If a project is going badly wrong, there should be a willingness to terminate it before completion. Action of this kind can be justified if the cost of the project escalates above earlier estimates or if the benefits expected from it are not likely to be realised. An attitude that, once work on a project commences, it must be completed regardless of changed circumstances, is to be avoided. Before making a final decision to terminate a project that is not going according to plan, the costs of termination (for example, payments that might have to be paid by way of compensation to contractors etc.) should be ascertained and made known to the appropriate authorities.'

Source: Ireland's Public Spending Code

http://publicspendingcode.per.gov.ie/

196. Monitoring and control of cost escalation is a serious concern for the MPI. From discussions and from reviewing the sample of transport projects, it is apparent that, for major infrastructure projects, cost control during design and implementation is a significant monitoring issue. Designed in the context of similar problems, South Korea's Total Project Cost Management system (TPCM), as described in Box 22 (and by extension in Box 20), provides a good international example of a responsive monitoring system geared towards cost control. It involves strict procedures for adjusting costs, as well as triggers for automatic review of the case for the project should cost increases become excessive (see Box 20) - something which is missing from Vietnam's monitoring and adjustment system. Some features of the TPCM system that are particularly worth noting are:

- The system only applies to major projects.

113 Article 99 on Rights and Responsibilities of Investors in Programs and Projects indicates one of the rights as being 'Decide to adjust, suspend or cancel the programs and projects'. 
- Allowance for a generous contingency is made, but there are very strict rules about adjustment thereafter.

- The minister responsible for planning and finance has the power to cut off funding to projects violating the TPCM guidelines

- Cost increases beyond a pre-specified tolerance trigger a fundamental reassessment of the project, which can, and does, lead to project cancellation.

- The performance of the PIM system itself is monitored - allowing assessment of the benefits of the system and identification of areas for improvement - as well as the projects within it.

\section{Box 22: South Korea's Total Project Cost Management System}

South Korea has a specialised budget management system for major public investment projects known as the Total Project Cost Management System (TPCM), which is based in the Ministry of Strategy and Finance (MoSF - the country's combined planning and finance ministry).

Established in 1994, it has been continually upgraded through annual revisions to the governing regulations. The TPCM is a tool for centralised monitoring of public investment expenditure and for identifying, checking and controlling increases in project costs throughout the project cycle from planning/design to completion of construction. It involves: i) systematic reporting on costs and cost changes; ii) external review of costs at key junctures; iii) monitoring of costs by the MoSF; and iv) strict protocols for making adjustments to costs. If costs change by more than specified tolerances, then a fundamental project re-assessment (Re-assessment of Feasibility Study - RFS) is triggered as indicated in Box 20.

The scope of the TPCM, which is governed by the National Finance Act (the equivalent of Vietnam's SBL), is:

- Projects whose construction period exceeds two years; and

- Civil engineering works whose TPC exceeds 30 billion Won (USD 30 million), or architectural and information systems projects whose TPC exceeds 10 billion Won (USD 10 million); and

- Projects implemented by the central government or its agents, or by local governments or private institutions that include central government funding.

The TPCM guidelines, as issued by the MoSF, regulate in detail the reporting, reviewing and adjusting of project costs at each phase of planning and design, at contracting and during construction. The guidelines require independent value-engineering experts to be consulted at both draft design and blueprint design phases. If there is any major change in design specification at blueprint design stage, this needs to be discussed and agreed with the MoSF. Any increase in costs at contracting phase compared to blueprint design needs to be discussed with the procurement management authority. The guidelines also include general and sector-specific rules and procedures for project adjustment (costs and specifications) during implementation.

There are some important rules associated with the TPCM, which balance operational flexibility with empowerment of the MoSF to control costs:

- Total project costs cover all cost items up to project completion, including design, land acquisition and construction, regardless of the source of funding.

- The base cost for the TPCM is the contract cost determined during bidding, which may be higher or lower than the cost estimate at the design phase.

- Construction contingencies of up to $8 \%$ of contract price can be budgeted to cope with the inevitable modifications during implementation.

- In general, cost increases through an increase in the size of a project are not allowed, except in unavoidable cases, which must be agreed with the MoSF.

- When a project under implementation violates the TPCM guidelines, the MoSF may cut off or withhold budget allocations for the project.

Source: The Power of Public Investment Management: Country Case Study of Rep. of S. Korea, Jay-Hyung Kim, World Bank, 2012. 
197. The recently established framework for monitoring does not make provision for riskbased monitoring, as is the case in more advanced systems. Categorisation of projects in terms of delivery risk can be a useful way of presenting and using monitoring findings. One option is to classify projects in terms of the risk of severe delay or cost escalation, using a red-amber-green (RAG) traffic light system (see Table 12). This is how the authorities responsible for portfolio monitoring in the UK, New Zealand and Colombia present their findings. It allows identification and closer monitoring of high risk projects and, by calculating the number or high risk projects, gives a way of assessing the overall riskiness of the total portfolio and changes over time. Annual reports by the UK Infrastructure Authority, for example, present information on the changes in numbers of red, amber or green projects by comparison to the previous year as a performance measure for the government major project portfolio.

\section{Table 12: Red-Amber-Green Delivery Confidence Rating Used in the United Kingdom and New Zealand}

\begin{tabular}{l|l|}
\hline \multicolumn{1}{c|}{$\begin{array}{c}\text { Delivery Confidence } \\
\text { 'RAG' Rating }\end{array}$} & \multicolumn{1}{c|}{ Criteria for 'RAG' Rating } \\
\hline Green & $\begin{array}{l}\text { Successful delivery of the project on time, budget and quality appears highly } \\
\text { likely and there are no major outstanding issues that at this stage appear to } \\
\text { threaten delivery significantly. }\end{array}$ \\
\hline Amber-Green & $\begin{array}{l}\text { Successful delivery appears probably; however, constant attention will be } \\
\text { needed to ensure risks do not materialise into major issues threatening delivery. }\end{array}$ \\
\hline Amber & $\begin{array}{l}\text { Successful delivery appears feasible but significant issues already exist, requiring } \\
\text { management attention. These appear resolvable at this stage and, if addressed } \\
\text { promptly, should not present a cost/schedule overrun. }\end{array}$ \\
\hline Amber-Red & $\begin{array}{l}\text { Successful delivery of the project is in doubt, with major risks or issues apparent } \\
\text { in a number of key areas. Urgent action is needed to ensure these are addressed, } \\
\text { and whether resolution is feasible. }\end{array}$ \\
\hline Red & $\begin{array}{l}\text { Successful delivery of the project appears unachievable. There are major issues } \\
\text { with project definition, schedule, budget, quality and/or benefits delivery, which } \\
\text { at this stage do not appear to be manageable or resolvable. The project may } \\
\text { need re-scoping and/or its viability reassessed. }\end{array}$
\end{tabular}

Source: Annual Report 2014-15, Major Projects Authority (renamed Infrastructure Authority in 2016). 


\section{Summary Assessment of Project Adjustment}

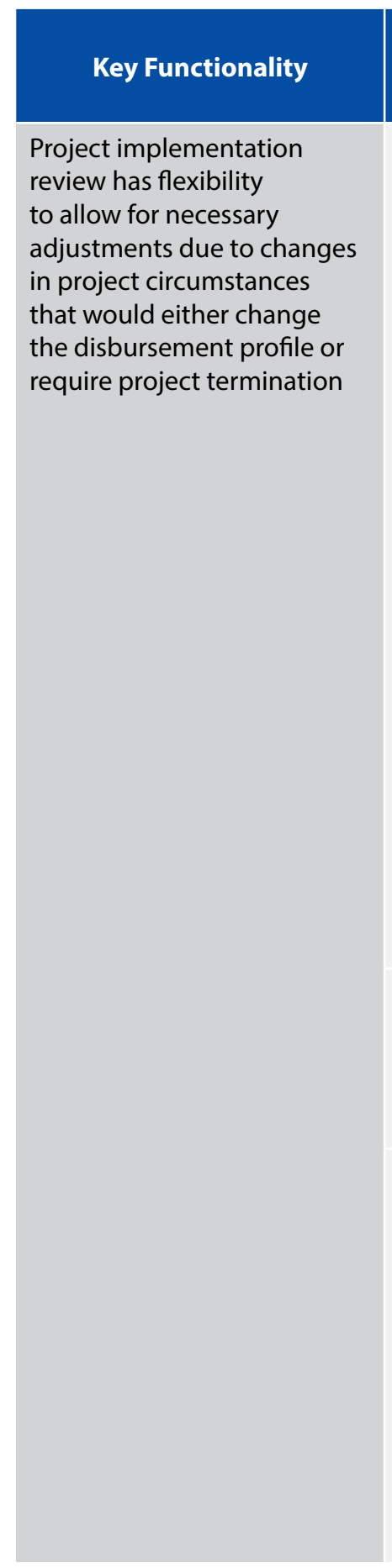

\section{Desirable Regulatory, Administrative and Technical Arrangements}

Responsive monitoring

Virement rules that balance efficiency with accountability

Procedures for fundamental review of projects that are seriously off-track and termination, where justified.

\section{Current Arrangements in Vietnam}

Active monitoring is largely decentralised, with the PMU monitoring the contractor, and the project owner monitoring the PMU. The MPI has been performing an inspection function, which, is largely compliance oriented, although it has some features of monitoring. For everyday implementation issues, this system serves its purpose well; however, responsive monitoring of the delivery of individual projects and of the portfolio of on-going projects as a whole is largely missing at the MPI level. This does not mean that there are no mechanisms for dealing with severe implementation difficulties, just that they are largely ad hoc, leading to reactions that are not necessarily timely. And there is no early warning of emerging difficulties. The new PIM information system created by the MPI offers the opportunity for it to undertake more active monitoring of problem projects and of the portfolio; however, instigating more active monitoring will rely on timely and reliable submission of electronic reports by project owners.

Budgetary rules are sufficiently flexible to allow budget holders to transfer funding, within limits, from slower moving to faster moving projects within the budget year, but the MPI must be consulted.

Technically there is a review process for project adjustments, but this does not distinguish between minor and major adjustments, and largely concerns technical feasibility and the realism of costings. Since the initial assessment of value for money at appraisal is insufficiently developed, there is no baseline against which to re-assess projects, in cases where costs have escalated or benefit projections slumped. The absence of a formal appraisal methodology also makes re-appraisal difficult. 


\section{Recommendations}

198. Strengthening of centralised monitoring is strongly indicated, with a focus on large-scale projects that are failing or at-risk of failing. This should be accompanied by systematic re-appraisal of problem projects, defined on the basis of quantitative triggers. Specifically, recommendations with respect to adjustment are to:

- Strengthen the role of the MPI in centralized monitoring of projects, with an emphasis on identifying and monitoring more closely major projects where the delivery risk is highest.

- Develop detailed guidelines on monitoring on the basis of Decree No. 84, elaborate supporting training materials and institute a training program, beginning at the central government level.

- Strengthen Decrees No. 59/2015 and 32/2015 to set out in more detail the requirements for fundamental review of projects requiring adjustment, allowing sufficient flexibility for minor adjustments and disciplined assessment of significant adjustments.

- Review relevant legal documents to harmonize the procedures for adjustments of ODA and non-ODA projects and remove the dual review process for adjustment of ODA projects.

- Review the PIL to make sure that it has adequate provisions for cancellation of failing projects by decision-makers on advice from public investment regulatory authorities. 


\section{Project Operation}

\section{What Does Good Practice Look Like?}

199. There are four features of a good process for sustainable operation of the new facilities created through capital investment projects:

- Formal handover of assets, including verification of fitness for purpose;

- Maintenance of comprehensive and up-to-date asset registers;

- Release of adequate financial resources for sustainable operations and maintenance; and

- Monitoring of service delivery.

200. A model PIM system includes the institutional arrangements necessary to ensure sustainable facility operation. The main purpose of much public capital investment is to deliver new or better public services or sustain the delivery of existing ones. As well as focusing on project management - whether projects are being delivered on time and to budget - a fully developed PIM system includes assessing the extent to which newly created assets are delivering the expected public services in the right quantity and at the right quality. A good system requires a formal handover of assets to those with management responsibility for operating and managing them. Verification that facilities are fit for the purpose intended, have not required significant adaptation or are not experiencing dramatic under-utilisation is also an important element of ensuring facilities are operating as planned. Adequate provision of financial resources for operations and maintenance is also vital for sustainable service delivery from a new facility (as noted in Section 8 on Selection and Budgeting). Once a facility is completed, it is good practice to continue monitoring service delivery - volumes and quality - to verify that the investment continues to perform as expected. This information can feed into a later impact assessment (see next section).

201. There needs to be a systemic process for registering new assets in appropriately informative asset registers, which are then kept up-to-date. Asset registers are a record of the property owned by the government and are an essential tool for planning of future public investments. A good asset management system can allow planners to understand the condition of public assets, the likely timeframe when they might need replacing (or capital renovation) and whether new requirements might be fulfilled by assets that already exist elsewhere and could be re-used. More sophisticated asset registers are also useful for understanding geographical areas of service coverage in important sectors such as healthcare and education. This information too can be used to inform investment decisions. Under-used or unwanted assets can be readily identified and can be sold off, with the receipts potentially going for investment in more productive assets. 


\section{Assessment of Project Operation in Vietnam}

There are disciplined procedures for project acceptance and handover, and assets are registered on the asset registers of operating entities. Shortfalls in funding for recurrent expenses are frequent, especially with respect to maintenance expenditures, which are consistently under-funded.

202. Vietnam has a well-regulated process for project acceptance and handover of newly created fixed assets. The quality of the process is confirmed by the completed projects (six in transport and one in education) in the project sample. Pre-acceptance tests are performed to ensure that assets are fit for purpose. Acceptance committees are created to formally accept the assets and transfer them to the users. Acceptance and handover are minuted by the acceptance committee at a formal meeting and the minutes serve as the legal confirmation. A project is first handed over to the project owner, e.g., the MOT in the case of a railway project, and then to the user/manager, e.g., Vietnam Railway Corporation. Acceptance committees are created by both parties. The handovers can occur concurrently or with some delay. If there is a delay, the project owner temporarily becomes responsible for operating and managing the asset. The Construction Law ${ }^{114}$ and associated regulations gives the legal basis for handover of construction works.

203. Fixed assets are recorded in asset registers. After handover to the managing and operating entity, the newly created asset is recorded in its asset register. Asset registers do not tend to be dynamic instruments with regular revaluations and assessments of the condition of the assets therein. Some efforts in this direction have been and are being pursued, notably in the roads sector, but this is not the general practice.

204. Maintenance of the stock of infrastructure assets, new and existing, is seriously underfunded in Vietnam leading to shortened operational lives, lower than anticipated benefits and reduced quality of public services. As already discussed in Section 8, there are no systematic linkages between the planning and budgeting of capital investment and the planning and budgeting of recurrent expenditure. As far as infrastructure sectors are concerned, this leads to underfunding of operations and maintenance, particularly the latter. Both budget allocations are typically below the established technical standards, and this is reinforced by releases typically falling short of allocations, as recurrent expenditure tends to suffer more in execution than capital (see Section 3). Ultimately, this compromises the planned useful life of assets. There is also work to be done on strengthening the realism of budget allocation norms for maintenance and major repairs. As an illustration, Box 23 gives indicative budgeting guidelines for maintenance from South Africa.

205. The transport sector and the roads subs-sector within it provide striking illustrations of underfunding of maintenance, but they are not alone in this respect ${ }^{115}$. Government transport strategies and plans often highlight maintenance as a priority, but this is not reflected in funding allocations. The World Bank has estimated that actual allocations for operations and maintenance only cover around $50 \%$ of what is necessary for transport infrastructure as a whole. For roads the situation is worse, with the Bank estimating that only $41 \%$ of the maintenance needs for the national road network were covered in 2013, which is in the middle of the $35-65 \%$ range for maintenance coverage over the period 2009-12. The result is a poor level of service - only $43 \%$ of roads in good condition ${ }^{116}$

114 Article 124 of the Construction Law, 'Handover of construction works'.

115 This discussion draws from the draft of a Public Expenditure Review currently under preparation by the World Bank.

$11637 \%$ in average condition and $20 \%$ in poor or very poor condition. 
- and higher costs stored up for the future ${ }^{117}$. Local roads are little different, with recent Bank work on piloting improved maintenance expenditure planning in five provinces indicating an average shortfall in maintenance spending of 57\%. Road maintenance costs are increasing in Vietnam because of rising input costs and wages, and if budgets do not move in proportion with these cost increases, the gap between needs and allocations will only widen.

206. Compared with other countries in the region and in the wider world, Vietnam devotes a far smaller proportion of total transport sector expenditure to maintenance ${ }^{118}$. The Bank estimates that only $10 \%$ of total road sector expenditure went to maintenance in $2012^{119}$, compared to $37 \%$ in Indonesia and 35\% in Malaysia for the same year. The OECD average was around $30 \%$ for 2011 . For the transport sector as a whole, maintenance expenditure has never exceeded $20 \%$ of the total, whereas a figure in the $20-30 \%$ range would be required to bring Vietnam into line with other countries and ensure assets last for their planned lives.

207. In sectors where charging for infrastructure services is usual, institutional arrangements are generally in place for full recovery of operating and maintenance costs through tariffs, but this is not always achieved in practice. In water supply, for example, legal instruments ${ }^{120}$ are in place for corporatisation of service providers and for charging of full cost recovery tariffs, i.e., tariffs that cover operating and maintaining expenditure and amortization. In sanitation, while cost recovery is the aim, the legal instrument ${ }^{121}$ leaves room for local governments to fill any gap between cost recovery tariffs and actual tariffs (which are determined by the Provincial People's Committee) with subsidies. Budgetary constraints caused by limited revenue raising capacities at local level, particularly in poorer provinces, mean that sometimes such subsidies are not always adequate, and maintenance of new and existing facilities suffers as a result. In localities, where donors are funding investment in water and sanitation, implementation of cost recovery tariffs is a condition of funding, so the risk of underfunding of maintenance is much lower. This is the case for World Bank and Asian Development Bank projects.

208. The situation with coverage of operations and maintenance costs resulting from asset creation in the social sectors is less evident ${ }^{122}$. The same lack of integration between capital and recurrent budget planning that besets the infrastructure sectors, together with an absence of systematic budget allocation mechanisms for recurrent spending, do not favour the rational planning of operations and maintenance expenditure for new facilities in the social sectors. As the World Bank has found in the education sector, the existing budget allocation mechanism for preschool and general education from the centre to provinces is not systematic - although notionally based on a formula it is subject to ad hoc adjustments - and does not provide incentives to provinces to plan and spend the education budget efficiently. This does not provide a good environment for ensuring coverage of the recurrent consequences of the investment decisions that come to fruition.

\footnotetext{
117 The World Bank estimates that, in general, repair costs six times maintenance costs after three years of neglect and 18 times after five years of neglect.

118 This discussion draws from the draft of a Public Expenditure Review currently under preparation by the World Bank

119 Average of $12 \%$ for $2009-13$.

120 Decree $117 / 2007 /$ ND-CP

121 Decree 80/2014/ND-CP

122 This discussion draws from the draft of a Public Expenditure Review currently under preparation by the World Bank.
} 


\section{Box 23: Indicative Budgeting Guidelines for Various Types of Infrastructure}

\begin{tabular}{|c|c|c|c|}
\hline $\begin{array}{l}\text { Type of } \\
\text { infrastructure }\end{array}$ & $\begin{array}{c}\text { Average } \\
\text { Annual } \\
\text { Maintenance } \\
\text { Budget as \% of } \\
\text { Repalacement } \\
\text { Cost }\end{array}$ & Key Assumptions & $\begin{array}{c}\text { Replacement } \\
\text { of Major } \\
\text { Rehabilitation } \\
\text { over and above } \\
\text { the Annual } \\
\text { Maintenance } \\
\text { Budget requiring } \\
\text { specific capital } \\
\text { budget }\end{array}$ \\
\hline Bulk water storage & $4-8 \%$ & $\begin{array}{l}\text { Mostly for periodic repair of electrical and } \\
\text { mechanical works, storm damage repair, routine } \\
\text { maintenance and periodic maintenance }\end{array}$ & $\begin{array}{l}\text { every } 30 \text { to } 50 \\
\text { years }\end{array}$ \\
\hline $\begin{array}{l}\text { Water treatment } \\
\text { works }\end{array}$ & $4-8 \%$ & Mostly for electrical and mechanical equipment & $\begin{array}{l}\text { every } 30 \text { to } 50 \\
\text { years }\end{array}$ \\
\hline Water reservoirs & $2-3 \%$ & $\begin{array}{l}\text { Generally low maintenance mostly of telemetry } \\
\text { and electrical equipment, storm damage repair, } \\
\text { pipe work repair, safety and security, routine } \\
\text { maintenance and periodic maintenance }\end{array}$ & $\begin{array}{l}\text { every } 20 \text { to } 30 \\
\text { years }\end{array}$ \\
\hline Water reticulation & $4-8 \%$ & $\begin{array}{l}\text { Mostly for telemetry and pumping equipment, } \\
\text { emergency leak repair and ongoing leak repair } \\
\text { due to degradation, storm damage repair }\end{array}$ & $\begin{array}{l}\text { every } 20 \text { to } 30 \\
\text { years }\end{array}$ \\
\hline $\begin{array}{l}\text { Sewage treatment } \\
\text { works }\end{array}$ & $4-8 \%$ & $\begin{array}{l}\text { Mostly for electrical and mechanical epuipment, } \\
\text { storm damage and periodic maintenance. }\end{array}$ & $\begin{array}{l}\text { every } 20 \text { to } 30 \\
\text { years }\end{array}$ \\
\hline Sewer reticulation & $4-8 \%$ & $\begin{array}{l}\text { Mostly for pumping equipment, emergency } \\
\text { leak repair and ongoing leak repair due to } \\
\text { degradation, blockage removal, storm damage } \\
\text { repair, }\end{array}$ & $\begin{array}{l}\text { every } 20 \text { to } 30 \\
\text { years }\end{array}$ \\
\hline $\begin{array}{l}\text { Roads and storm } \\
\text { water }\end{array}$ & $5-10 \%$ & $\begin{array}{l}\text { Mostly for emergency repair, storm damage } \\
\text { repair, and periodic maintenance (resurfacing } \\
\text { every } 7 \text { to } 10 \text { years). }\end{array}$ & $\begin{array}{l}\text { every } 20 \text { to } 30 \\
\text { years }\end{array}$ \\
\hline $\begin{array}{l}\text { Electricity } \\
\text { reticulation }\end{array}$ & $10-15 \%$ & $\begin{array}{l}\text { Mostly for emergency repair, storm damage } \\
\text { repair, safety and security, routine maintenance } \\
\text { and periodic maintenance. }\end{array}$ & $\begin{array}{l}\text { every } 20 \text { to } 30 \\
\text { years }\end{array}$ \\
\hline Public buildings & $4-6 \%$ & $\begin{array}{l}\text { Mostly for emergency repair, storm damage } \\
\text { repair, and periodic maintenance (e.g repainting } \\
\text { and cosmetic upgrades every } 5 \text { to } 10 \text { years) }\end{array}$ & $\begin{array}{l}\text { every } 30 \text { to } 30 \\
\text { years }\end{array}$ \\
\hline Hospitals & $5-8 \%$ & $\begin{array}{l}\text { Mostly for emergency repair, storm damage } \\
\text { repair, and periodic maintenance (e.g. (e.g } \\
\text { repainting every } 3 \text { to } 5 \text { years, and cosmetic and } \\
\text { operational upgrades every } 7 \text { to } 10 \text { years). }\end{array}$ & $\begin{array}{l}\text { every } 20 \text { to } 30 \\
\text { years }\end{array}$ \\
\hline Schools & $4-6 \%$ & $\begin{array}{l}\text { Mostly for emergency repair, storm damage } \\
\text { repair, and periodic maintenance (e.g. repainting } \\
\text { every } 5 \text { to } 7 \text { years). }\end{array}$ & $\begin{array}{l}\text { every } 30 \text { to } 50 \\
\text { years }\end{array}$ \\
\hline $\begin{array}{l}\text { Electricity } \\
\text { generation }\end{array}$ & $5-8 \%$ & $\begin{array}{l}\text { Mostly for electrical and mechanical equipment } \\
\text { and dependent on age and technology of works }\end{array}$ & $\begin{array}{l}\text { every } 30 \text { to } 50 \\
\text { years }\end{array}$ \\
\hline $\begin{array}{l}\text { Electricity } \\
\text { reticulation }\end{array}$ & $10-15 \%$ & $\begin{array}{l}\text { Mostly for emergency repair, storm damage } \\
\text { repair, safety and security, routine maintenance } \\
\text { and periodic maintenance (e.g. every } 7 \text { to } 10 \\
\text { years). }\end{array}$ & $\begin{array}{l}\text { every } 20 \text { to } 30 \\
\text { years }\end{array}$ \\
\hline
\end{tabular}

Source: Infrastructure Maintenance Budgeting Guidelines, National Infrastructure Maintenance Strategy, Republic of South Africa. 


\section{Summary Assessment of Project Operation}

\section{Key Functionality}

Process for ensuring that a facility is ready for service delivery should be in place, and asset registers are maintained and asset values recorded.

\section{Desirable Regulatory, \\ Administrative and Technical Arrangements}

Formal handover of assets, including verification of fitness for purpose

Maintenance of
comprehensive and up-to-
date asset registers

Provision of adequate resources for sustainable operations and maintenance

Monitoring of service delivery

\section{Current Arrangements in Vietnam}

There is a good process for acceptance and handover of newly created assets, which includes pre-acceptance testing of whether design specifications have been met and whether the facility is ready to begin operating as intended.

Assets are formally recorded in the asset registers of operating entities following handover. Asset registers tend to be static accounting tools, rather than dynamic tools for managing, maintaining and renewing an organisation's stock of assets.

Operations and maintenance are seriously underfunded in Vietnam's infrastructure sectors. This derives from the lack of integration when budgeting and the overall bias towards capital spending that pervades the system (as discussed in under Selection and Budgeting).

There is provision in the regulatory framework for formal monitoring of service delivery once newly completed assets begin operating, but this has not been properly operationalised yet.

\section{Recommendations}

\section{The sustainability of investment projects should be improved by increasing maintenance} allocations. The use of modern asset management tools should also be extended, beginning with the most important infrastructure sectors. Detailed recommendations that emerge from the preceding analysis of operations are to:

- Extend the international benchmarking of operations and maintenance expenditure, beyond the transport sector, to get a better picture of where Vietnam stands.

- Increase maintenance spending as a share of total spending in infrastructure sectors towards levels more commonly found in better performing peers.

- Explore the opportunities for greater use of asset management tools, as is already being done in the roads sector on a trial basis.

- Operationalise monitoring of service delivery performance on completion of projects. 



\section{Project Evaluation}

\section{What Does Good Practice Look Like?}

\section{There are three desirable institutional arrangements for a good process for evaluation of completed projects:}

- Availability of a policy and methodological guidance on the performance of ex post studies;

- Basic completion review for all projects carried out soon after completion; and

- Impact evaluations undertaken for all major projects and a sample of smaller projects.

\section{A properly functioning PIM system requires a systematic review of projects once completed as a basis for lesson learning - from both successes and failures - at both project and strategic levels. In its simplest form, this would be a project completion report that verifies whether a project has been completed on time, to budget and to specification. To be useful though, this needs to be more than a certification process: as well as identifying departures from plan, a project completion report should analyse the reasons for these departures and suggest corrective action applicable to future projects. The completion report would also include the results of an analysis of service delivery surveys, where this can be assessed.}

\section{Box 24: Ireland's Approach to Ex Post Evaluation}

\section{Post-Project Review}

"All large capital projects and a proportion of other capital projects have to be subjected to a post-project review to see if the predicted benefits of the project were realised. Post-project reviews should be undertaken once sufficient time has elapsed to allow the project to be properly evaluated with sufficient evidence of the flow of benefits/costs from it. There are two separate focuses of review - (i) project outturn and (ii) appraisal and management procedures. The second element can be done after project completion as it involves reviewing administrative and management procedures. The timing of the first element will depend on the nature of the project i.e. the period required to observe the expected benefits. This period should be no longer than one third of the timeframe used in the Appraisal. The detailed appraisal provides the base against which the outturn review is made. The aim of a review of project outturn is to determine whether:

- The basis on which a project was undertaken proved correct;

- The expected benefits and outcomes materialised;

- The planned outcomes were the appropriate responses to actual public needs;

- The appraisal and management procedures adopted were satisfactory;

- Conclusions can be drawn which are applicable to other projects; to the on-going use of the asset; or to associated policies.

\section{Mandatory Evaluation/Post-Project Review Requirements}

- $\quad \ldots$

- All Capital Projects costing $>€ 20 \mathrm{~m}$ are to be subject of a post-project review

- At least $5 \%$ of other capital projects should be reviewed

- $\quad \ldots$ 


\begin{abstract}
Additional Evaluation/Post-Project Review Requirements
Departments and agencies should not restrict themselves to the mandatory evaluation or post-project review requirements. From time to time it may be apparent that while not mandatory, an area of expenditure would benefit from a more in-depth review based on the picture the performance indicators paint or maybe because the performance indicators are not as informative as originally thought.
\end{abstract}

\title{
Communicating lessons learned
}

As with all parts of the Public Spending Code any significant lessons should be translated into changes in the Sponsoring Agency's practices and communicated within the organization and to the sanctioning authority so that it can apply any general lessons learned to this Code or to supplementary information.

\section{Responsibility for Evaluation/Review}

It is the responsibility of the Sponsoring Agency to carry out the evaluations or post project reviews. Those conducting reviews and evaluations should not be the same people as conducted the appraisal or managed the implementation.

Source: Infrastructure Maintenance Budgeting Guidelines, National Infrastructure Maintenance Strategy, Republic of South Africa.

212. In its more advanced form, systematic review extends to an impact evaluation of whether a project has delivered the forecast social benefits, whether value for money has been achieved and whether the achieved impact has contributed to achieving national policy priorities. In the language used in evaluation and performance budgeting, impact assessments look the achieved efficiency, effectiveness and policy relevance of the project. This might involve re-running any social cost-benefit analysis, on the basis of achieved costs and benefits rather than forecasts, and assessing whether demand for services, e.g., traffic using a new road or users of new social facilities, actually materialised as forecast. In-depth evaluations, are probably best done on a sample basis, rather than for all projects. Evaluation would normally be conducted some years after completion so as to allow sufficient time for impacts to be assessed and attributed to a project. To ensure objectivity, impact evaluation is best performed by independent evaluators. Ireland represents an example of good practice in impact evaluation (referred to as post-project review) as illustrated in Box 24.

\section{Assessment of Project Ex Post Evaluation in Vietnam}

While Vietnam has now established a firm legal and regulatory basis for ex post evaluation, impact assessments are rarely performed, except for ODA projects, and lesson learning is not a strong feature of completion reports.

213. Although already reasonably well defined ${ }^{123}$, the legal and regulatory framework for ex post evaluation has recently been put on a firmer footing in Vietnam. The PIL establishes the legal basis for evaluation ('assessment'), identifying the different types of assessment - initial assessment, mid-term assessment, final or stage assessment, impact assessment and 'extraordinary' assessment ${ }^{124}$ - and setting out their basic contents ${ }^{125}$. Decree No. 84/2015/ND-CP, governing supervision and assessment, goes on to further define responsibilities and basic procedures for these assessments, as well as establishing their timing more clearly. According to the allocation of responsibilities, investors

123 Decree No. 13/2009/ND-CP

124 In other systems, these might be known as conditional assessments, because they are triggered by the occurrence of certain conditions.

125 Articles 81 and 82 of the PIL 
carry out initial assessments, mid-term assessments and final assessments, and investment decisionmakers perform impact assessment and extraordinary assessments of the projects under their management. As the investment-decision maker changes with the category of project, responsibility for the impact assessment will also shift. The provision made in the regulatory framework for initial assessment (immediately before implementation) and mid-term assessment is good practice for major projects.

\section{The types of assessment defined in the legal and regulatory framework are largely} consistent with international practice. The final assessment equates to an advanced completion review, covering delivery of benefits ${ }^{126}$, and the impact assessment can be seen as broadly conforming to an ex post evaluation of efficiency, effectiveness and impact ${ }^{127}$ (see Box 26 for a description of the kind of analysis that should be expected in such a study). Mid-term evaluations are also common for major projects or staged projects in good practice countries. Also in common with international practice, certain types of assessment are reserved for the most important projects: projects of national importance and Group A projects require all types of assessment, whereas Group B and C only require terminal assessment and impact assessment. Many countries only carry out impact assessments of the largest projects and a sample of less important projects (see the case of Ireland in Box 24 above), so the requirement for all Group $B$ and $C$ projects to have impact assessments is onerous by international standards.

215. Completion reports are prepared but are not always systematic in analysing implementation problems and identifying lessons. ODA projects are consistent in their requirements for analytical completion reports, with an emphasis on drawing out lessons from deviations from plans. For fully domestically funded projects, the practice is more variable. This being said, one of the completed projects in the sample of transport projects did have an analytical completion report prepared. There is a difference between ensuring that a project is fit for purpose - which tends to be the focus of completion reports at present - and assessing implementation performance with a view to making improvements in future projects. Both are important activities, but the latter needs to be more analytical, exploring reasons for successes and failures, and deciding what lessons might be applicable for future projects. Box 25 gives an example of a format for a completion report. There is scope for improving this aspect of completion reports in Vietnam, in line with the requirements embodied in the legal and regulatory framework. This will require the preparation of detailed methodological guidance and accompanying training.

126 According to the decree, a final assessment means an 'assessment carried out right after completion of the investment project in order to examine the results and draw valuable lessons'.

127 According to the decree, an impact assessment means an 'assessment carried out at an appropriate time after the third year from the day on which the project is put into operation in order to clarify the effectiveness, sustainability, and socio-economic impact compared to initial targets'. 


\section{Box 25: Format for a Project Completion Report}

1. General Project Information

1.1 Background

1.2 Post-Implementation Review Objectives and Scope

1.3 Post-Implementation Review Team Members

2. Findings

2.1 Management Effectiveness

2.2 Cost

2.3 Schedule

2.4 Unplanned Side-Effects

2.5 Residual Risks

3. Administrative Closure and Accomplishments

3.1 Performance Metrics

3.2 Customers' and Users' Satisfaction

4. Open Issues

5. Lessons Learned

5.1 Recommendations

6. Contract Closure

Source: 'Project Completion Report: PPM Version 2.0' US Department for Housing and Urban Development, 2015

216. Although there is a legal framework in place, the reality is that little in the way of classic ex post evaluation of project impacts and subsequent lesson learning is currently taking place as far as domestically funded projects are concerned. This is partly explained by the newness of the framework and the fact that relatively few projects have been begun and completed under the PIL. All the same, if the framework is to be put into practice, it will require a dedicated effort, involving development and circulation of detailed methodologies for the various types of assessment, together with training in the implementation of these methodologies. This still remains to be done. In addition to limited technical guidance and training, lack of financial resources to carry out evaluations is also an issue. It is standard practice for donors and IFls to undertake completion reviews and ex post evaluations of the projects that they have funded. 


\begin{tabular}{|c|c|c|}
\hline \multicolumn{3}{|c|}{ Box 26: Qualitative and Quantitative Evaluation Criteria } \\
\hline \multirow[t]{5}{*}{$\begin{array}{l}\text { Strategic Performance } \\
\text { Evaluation Criteria }\end{array}$} & Efficiency & $\begin{array}{l}\text { Measures operational success: To } \\
\text { what degree have the outputs } \\
\text { achieved derived from efficient } \\
\text { use of financial, human and } \\
\text { material resources? }\end{array}$ \\
\hline & Effectiveness & $\begin{array}{l}\text { Measures tactical success: Were } \\
\text { the stated objectives achieved } \\
\text { and to what extent did the } \\
\text { project contribute to the goal } \\
\text { achievement? }\end{array}$ \\
\hline & Impact & $\begin{array}{l}\text { Measures strategic success: Has } \\
\text { the project had any other positive } \\
\text { or negative consequences other } \\
\text { than those planned? }\end{array}$ \\
\hline & Relevance & $\begin{array}{l}\text { Measures strategic success: Has } \\
\text { the project been in line with the } \\
\text { needs and priorities of the owners, } \\
\text { the intended users and other } \\
\text { affected parties? }\end{array}$ \\
\hline & Sustainability & $\begin{array}{l}\text { Measures strategic success: Are the } \\
\text { positive effects derived from the } \\
\text { project likely to continue after the } \\
\text { project has been completed? }\end{array}$ \\
\hline \multicolumn{2}{|c|}{ Socio-Economic Profitability Criterion } & $\begin{array}{l}\text { Has the project delivered a } \\
\text { positive net present value? }\end{array}$ \\
\hline
\end{tabular}

\section{The underdeveloped status of ex post evaluation in Vietnam should not be considered as}

unusual. This is often the last stage of the PIM system to be fully developed, although a few countries, e.g., Ireland, have led PIM reform through a rigorous evaluation process, using it to drive reforms at earlier stages. Generally, improvements in planning and appraisal are the forerunner of better impact evaluation, because it is important to have well defined objectives - at outcome and intermediate outcome level, not just outputs - and the results of social cost-benefit analysis, as a starting point for assessing actual project achievements, compared to targets and projections. A staged development of evaluation is appropriate, moving in line with improvements in other parts of the PIM system. Also, while it is not unusual for project owners to be responsible for evaluations (as is required by Vietnam's legal and regulatory framework), procedures for independent review and quality control of completed evaluations are advisable ${ }^{128}$. Such procedures are not presently foreseen. This function that would be best performed by the public investment regulatory agencies, the MPI and provincial DPIs.

128 It is common practice to contact out evaluation to consultants specialising in this field. This is also foreseen in Vietnam's legal framework. While consultants are meant to give independent advice, they are contracted by the project owner, which could introduce an element of bias. 


\section{Summary Assessment of Project Evaluation}

\begin{tabular}{|c|c|c|}
\hline Key Functionality & $\begin{array}{l}\text { Desirable Regulatory, } \\
\text { Administrative and Technical } \\
\text { Arrangements }\end{array}$ & Current Arrangements in Vietnam \\
\hline \multirow[t]{3}{*}{$\begin{array}{l}\text { Basic completion } \\
\text { review and ex } \\
\text { post evaluation of } \\
\text { completed projects } \\
\text { are conducted. }\end{array}$} & Policy and guidance on ex post review & $\begin{array}{l}\text { The requirement for evaluation of } \\
\text { completed projects is foreseen in the legal } \\
\text { and regulatory framework for PIM, but the } \\
\text { detailed methodological guidance needed } \\
\text { to put this requirement into practice is } \\
\text { missing. }\end{array}$ \\
\hline & $\begin{array}{l}\text { Basic completion review for all } \\
\text { projects }\end{array}$ & $\begin{array}{l}\text { Current reporting on project completion } \\
\text { falls short of a full project completion } \\
\text { review, which should have a focus on lesson- } \\
\text { learning, and does not feed into an organised } \\
\text { structure for disseminating lessons. }\end{array}$ \\
\hline & $\begin{array}{l}\text { Impact assessment of major projects } \\
\text { and a sample of smaller projects }\end{array}$ & $\begin{array}{l}\text { Impact assessments, looking at efficiency, } \\
\text { effectiveness and sustainability, are foreseen } \\
\text { in the legal and regulatory framework, but } \\
\text { are not systemically undertaken, except } \\
\text { for ODA projects. Impact assessments of } \\
\text { all projects are foreseen, but this may be } \\
\text { too heavy a requirement for less significant } \\
\text { projects. }\end{array}$ \\
\hline
\end{tabular}

\section{Recommendations}

218. In general it can be said that there are very significant opportunities for improving systems for lesson learning from project implementation experience. Looking to the longer term, impact evaluation should be operationalised, but on a phased basis given the current status. More specifically, it is recommended that the authorities:

- Develop a more systematic lesson learning process based on an analysis of project implementation performance. This would involve an extended project completion report format, guidance on implementing performance analysis and lesson learning, and establishment of a structured approach to disseminating lessons.

- In the short - to medium-term, carry out a pilot ex post evaluation of a small number of major projects - perhaps some successful projects and some more problematic - as a basis for developing a replicable methodology. The methodology should include assessment of efficiency, effectiveness, impact, relevance and sustainability.

- In the medium - to longer term, roll-out systematic evaluation of the impacts and strategic relevance, i.e., contribution to achievement of SEDP priorities, of completed projects. This is a longer term priority because it will require significant capacity building in performance evaluation and also because evaluation relies on solid project planning, including the identification of monitoring and evaluation indicators against which performance can be judged. Few countries carry out evaluations of all projects, but usually a sample is taken. This is the recommended approach for Vietnam, probably beginning with the largest or most problematic projects.

- Develop procedures for independent review and quality control of completed ex post impact evaluations. 


\section{Key Constraints on PIM System Functioning}

219. Vietnam has made commendable progress in reforming its PIM, bringing it from a central planning system to notably closer to international good practice. Diversified modalities of public capital spending are specified and classified into distinct project categories to facilitate the on-going decentralization and effective management. The legal framework to govern PIM has continuously improved, and now covers the entire project management cycle. The recent promulgation of the $\mathrm{PIL}$ and the amended $\mathrm{CL}$, as well as the associated guiding by-laws have established sound legal foundations for an effective PIM system.

220. There is, nevertheless, significant room for improvement to enhance both the allocative and productive efficiency of public investment in Vietnam, so as to provide high quality infrastructure services and crowd in private investment. In identifying PIM as one of the main reform priorities for 2011-20, the Government of Vietnam has been working on the formulation of a well-designed PIM reform program to enhance investment efficiency and productivity.

221. This report has aimed to contribute to the formulation of this reform program and has analysed Vietnam's PIM system using the WBG's assessment framework, which draws attention to eight 'must-have' features of a functioning system. On the basis of the assessment in previous sections, the critical weaknesses in PIM system performance in Vietnam are judged currently to be:

(i) Vietnam has a large number of strategic documents but they lack the coherence and realism necessary to provide strategic guidance for public investment in Vietnam, and first-level screening has a narrow scope;

(ii) The practical status of project appraisal in the broader system remains ambiguous, and elements of good practice in project appraisal have not yet been fully operationalized;

(iii) The necessity for some form of independent review of project proposals is recognized in the legislation, but the design falls short of a fully impartial review of the feasibility study and appraisal findings;

(iv) Criteria/principles for selecting projects for budget funding are defined in the State Budget Law and PIL, but they are not very precise, and lack a strong policy orientation, as far as selecting new projects is concerned, and their application is, in practice, not very transparent or consistent;

(v) Project management arrangements in Vietnam are generally reasonably robust, but there is scope for further strengthening and addressing weaknesses in application, particularly at the province level;

(vi) Further work needs to be done so that the MPI is in a position to be able to identify projects with a high risk of delivery failure and then to ensure that adequate steps are being taken to bring them back on track and, in the worst cases, close them down;

(vii) Shortfalls in funding for recurrent expenses are frequent, especially with respect to maintenance expenditures, which are consistently under-funded; and 
(viii) While Vietnam has now established a firm legal and regulatory basis for ex post evaluation, impact assessments are rarely performed, except for ODA projects, and lesson learning is not a strong feature of completion reports.

\section{Cross-Cutting Issues}

222. Some of the recommendations of this report will mean a stronger role for the MPI, and in some instances for the MOF. In particular, the wider scope of scrutiny at first-level screening (investment intention decision), independent review of the basis for investment decisions and strengthened central monitoring will all require a deeper involvement from the MPI. At first-level screening, the MPI will be expected to examine the strategic relevance and rationale for projects, as well as affordability. Independent review of feasibility studies and appraisal reports by a disinterested party is a critical safeguard against optimism bias. Centralised, risk-based monitoring is important for getting poorly performing projects back on track or for ensuring that reassessment is triggered in extreme cases. In addition, a central body needs to coordinate and promote lesson learning from ex post review, once established. These are all critical functions, which the MPI is best placed to perform.

223. Reinforcing and broadening the role of the MPI must be carefully designed and sequenced so that new tasks can be performed efficiently and effectively, without hindrance to the smooth running of the PIM system as a whole. This will mean designing in detail and then piloting improved processes before their full enactment, rather than regulating for change before the necessary procedures and tools are in place and tested. It will also require developing the necessary capacities before full implementation, so that administrative bottlenecks are not created, putting the reforms into disrepute. Defining human capacity needs and assessing gaps, both in terms of numbers and capabilities, should come first, followed by design and implementation of a realistic capacity building program.

224. The envisaged role for the MPI is deliberately contained to large-scale projects, since central agencies cannot realistically be directly involved in all projects because of capacity and time limitations. For lower value projects, the MPI's role should be one of developing systems and methods, and overseeing and supporting their application. 


\section{Summary of Recommendations by PIM Function}

\section{The following table presents a comprehensive set of reform recommendations} organised around the eight key functionalities of the WBG assessment framework. Headline recommendations are followed by recommendations on specific actions, for which the responsible entities and timing are indicated. These recommendations summarise the more detailed recommendations given at the end of each section of the preceding assessment.

\begin{tabular}{|l|c|c|} 
Recommendation & $\begin{array}{c}\text { Lead } \\
\text { Responsible } \\
\text { Entity }\end{array}$
\end{tabular}

\section{Strategic Guidance and First-Level Screening}

- Improve long term strategic planning of major infrastructure

- Reinforce the role of the MPI and mainstream the MOF's review in first-level screening (investment intention stage) of large-scale projects with significant fiscal impacts.

- Ensure system design supports the reversibility, at appraisal, of a positive investment intention decision.

1. Examine options for developing a rolling long-term infrastructure strategy for major projects.

MOT, MOF, MPI Medium term

MPI Medium term beyond appraising affordability of large-scale project proposals.

3. Streamline and standardize MOF review of repayment capacity and ensure transparency and adequate timing of the review process, notably with respect to ODA projects.

4. Introduce looser conditions for including ODA projects in the outer years of the MTIP

5. Introduce a decision step for major projects prior to preparation of a pre-feasibility study.

6. Make the investment intention decision a decision to proceed to appraisal, which is reversible at appraisal.

7. Develop methodological guidance on Pre-Feasibility Study and Report on Investment Intentions.

\section{Project Appraisal}

- Make the investment decision the critical decision of the 'quality-at-entry' process

- Create the necessary regulatory, technical and capacity conditions for making social cost-benefit analysis the default analytical tool for appraisal decisions

1. Raise the status of the investment decision in legal and practical terms, and start measuring system performance for this stage.

2. Review and revise the legal and regulatory framework to make social cost-benefit analysis the default analytical tool, with defined exceptions and alternative tools.

3. Develop and issue standard methodological guidance for appraisal, including social cost-benefit analysis and alternative tools.

4. Estimate standard parameter values for use in social costbenefit analysis, beginning with the social discount rate.

\section{MPI Medium term}

MPI and MOC Medium term

MPI and MOC Short to medium term

MPI, MOC, MOT, MARD, MOIT
Short to medium term 


\section{Recommendation}

5. Assess capacity building needs and implement continuous training in appraisal methods.

6. Review the parallel systems for appraisal of construction and non-construction projects and develop a uniform process.
Lead

Responsible Entity

MPI, MOC, MOT, MARD, MOIT

MPI and MOC

\section{Timing}

Short to medium term

Short term to medium term

\section{Independent Review}

- Design and implement a strengthened independent review function, focusing on large-scale projects

1. Develop and apply criteria for reviewing feasibility studies. MOC, MPI, MOF Short term

2. Amend the $\mathrm{CL}$ to strengthen the role of the MPI in independent review, especially for large projects with significant impacts.

3. Assess capacity building needs of MPI and MOC and implement training required to take on an enhanced role in independent review.

4. Develop a database of projects - ideally integrated with the PIM information system - to track and manage the 'qualityat-entry' stages of PIM.

MPI, MOC Medium-term

MPI, MOC Short to medium term

MPI, MOF Short to medium term

\section{Selection and Budgeting}

- $\quad$ Situate project selection and budgeting in a realistic medium-term budgetary perspective.

- Move towards a budgeting process which locks in funding for on-going projects before allocating to new projects.

- Integrate more closely the budgeting of capital and recurrent expenditures.

1. Develop principles/criteria for selecting new projects in annual investment plans.

MOF, MPI Short term

2. Introduce the preparation of forward capital baseline estimates as a first step in capital budgeting

3. Design and introduce a credible rolling, medium-term budget framework, which is attuned to the needs of PIM.

MPI, MOF Short term

4. Carry out a 'clean-up' of the portfolio of on-going public investment projects.

5. Introduce a two-step capital budgeting process, where funding for on-going projects is decide before budgets for new projects are allocated

6. Assign a formal 'gatekeeping' function to the MPI, allowing the rejection of non-compliant projects

MOF Short to medium term

. Require budget requests for investment projects to include projections of future operating and maintenance expenditures.

\section{Project Implementation}

- $\quad$ Strengthen project management arrangements.

- Improve value for money in procurement by making greater use of competitive procurement methods.

1. Develop detailed project management instructions/ guidance.

MOC, MPI, MOF Short to medium term

2. Introduce a requirement for a project implementation management plan.

MOC, MPI Medium term 
3. Institutionalise project steering committees for major projects.

MPI Short term

MOC Short term view to harmonisation.

5. Reduce the use of restrictive procurement practices and increase the use of competitive procurement.

6. Proceed with the development of the PIM information system, prioritising linkages with TABMIS

\section{Project Monitoring and Adjustment}

- Strengthen centralised monitoring, with a focus on large-scale projects that are failing or at-risk of failing.

- Systematically re-appraise problem projects defined on the basis of quantitative triggers.

1. Strengthen the role of the MPI in centralised monitoring. MPI Short to medium term

2. Develop detailed instructions/guidelines on monitoring (and evaluation).

3. Develop and implement training in monitoring (and evaluation) based around new guidelines

\begin{tabular}{|c|l|}
\hline MPI & Short to medium term \\
\hline MPI, MOC & Short term \\
\hline MPI, MOC & Short to medium term
\end{tabular}

4. Review the regulatory framework for the Construction Law to make fundamental review more effective, by requiring reviews only when costs or benefits rise/fall by a significant proportion (which should be defined in quantitative terms).

MPI and MOC Short to medium term

5. Improve the regulatory framework to make it easier to cancel failing projects, taking account of cancellation costs.

6. Harmonise arrangements for adjustment of ODA and nonODA projects

\begin{tabular}{|l|l|}
\hline MPI & Short term \\
\hline MPI & Medium term
\end{tabular}

\section{Project Operations}

- Improve the sustainability of investment projects by increasing maintenance allocations.

- Extend the use of modern asset management tools, beginning with the most important infrastructure sectors.

1. Extend international benchmarking of Vietnam's budget allocations for maintenance.

2. Increase the share of maintenance spending as a share of total expenditure in line with international comparisons.

3. Explore the greater use of advanced asset management systems - using modern information collection and processing methods - in infrastructure sectors.

4. Operationalise monitoring of service delivery performance on completion of projects.

\begin{tabular}{|l|l} 
MOF & Short term \\
\hline MOF & Short to medium term \\
\hline MOF & Medium term \\
\hline MOF & Medium to long term
\end{tabular}




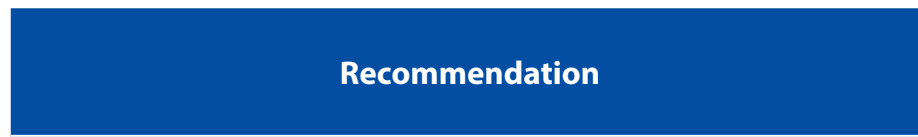

\section{Lead \\ Responsible Entity}

\section{Timing}

\section{Ex post Evaluation}

- Improve systems for lesson learning from project implementation experience

- Operationalise impact evaluation on a phased basis

1. Design and implement procedures for systematic lessonlearning, based on a more analytical basic completion report.

MPI, MOC Short term

2. Pilot ex post impact evaluation for a small number of projects.

3. Develop an evaluation methodology based on the results of piloting.

4. Roll-out application of the evaluation methodology, supported by training programme.

5. Develop arrangements for independent review of evaluations.

\begin{tabular}{l|l} 
MPI & Short to medium term
\end{tabular}

MPI Medium term

MPI Long term

MPI Long term 


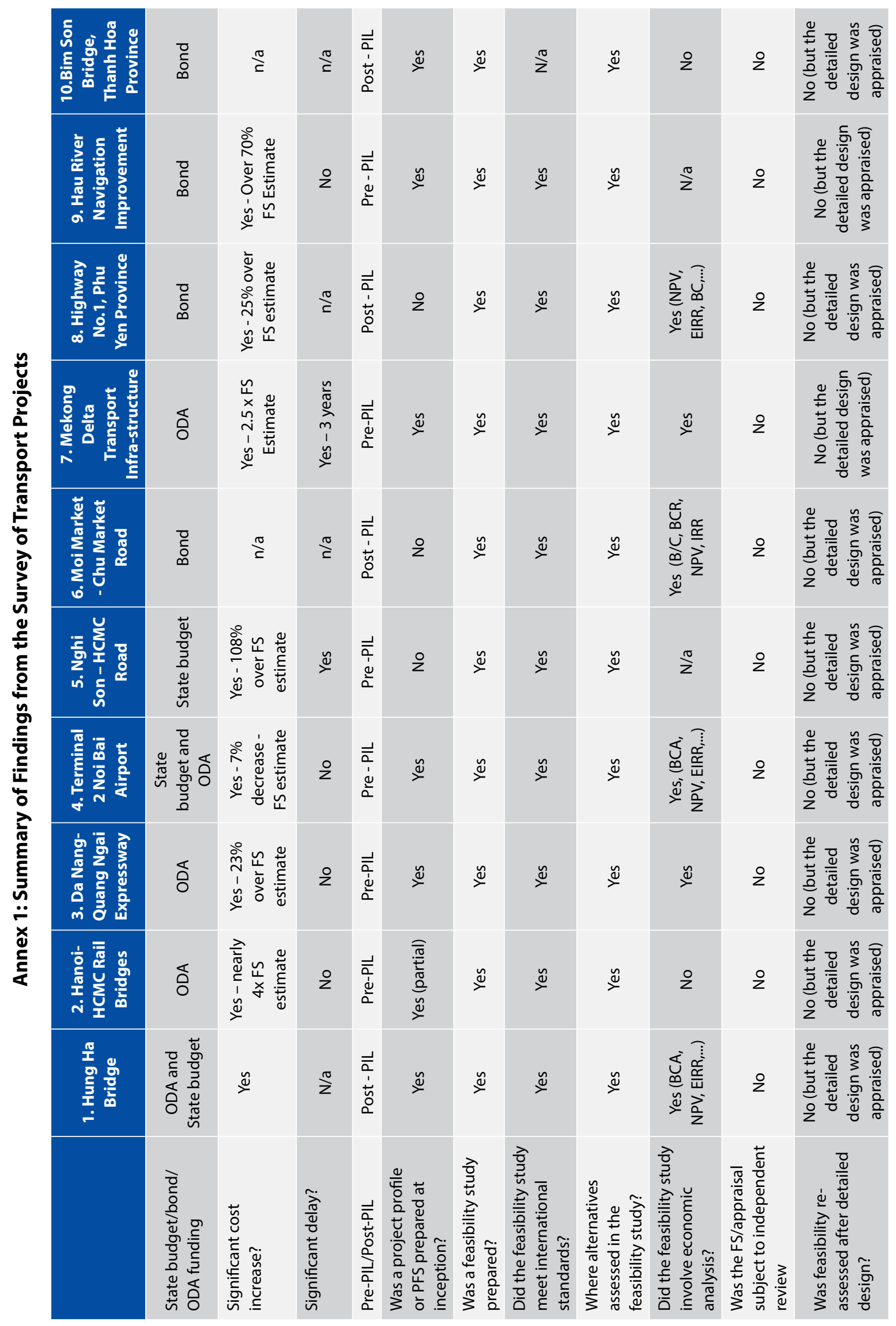




\begin{tabular}{|c|c|c|c|c|c|c|c|c|c|}
\hline 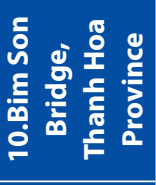 & 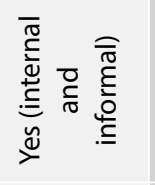 & $\stackrel{\check{\varpi}}{=}$ & 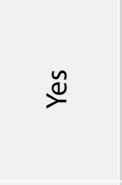 & 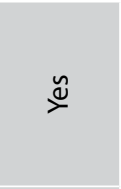 & $\stackrel{\check{\Xi}}{=}$ & 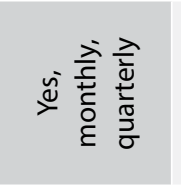 & $\stackrel{\pi}{z}$ & $\frac{\pi}{z}$ & $\frac{\pi}{z}$ \\
\hline 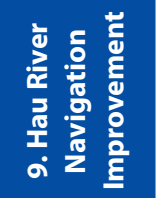 & 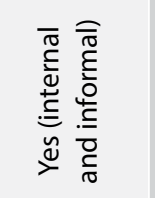 & 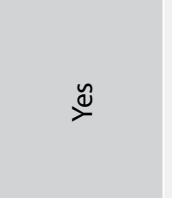 & $\stackrel{\Perp}{\nu}^{\check{n}}$ & $\stackrel{\check{\varpi}}{=}$ & $\stackrel{\check{\Perp}}{\check{\nu}}$ & 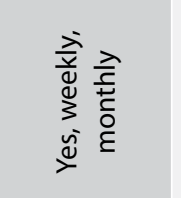 & $\stackrel{\check{\Xi}}{\underline{\nu}}$ & 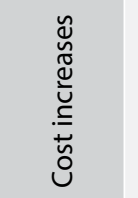 & $\stackrel{\circ}{2}$ \\
\hline 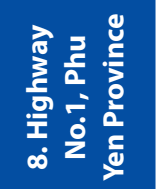 & 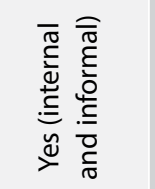 & 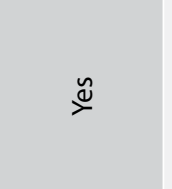 & 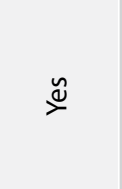 & $\stackrel{\check{\varpi}}{\check{\nu}}$ & $\stackrel{\widetilde{\nu}}{\check{\nu}}$ & 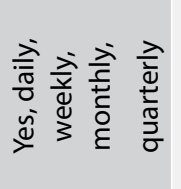 & $\stackrel{\check{\varpi}}{\check{\nu}}$ &  & $\stackrel{0}{2}$ \\
\hline 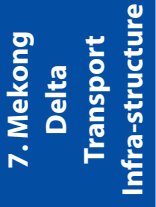 & 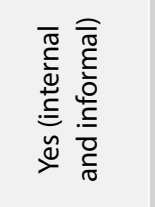 & 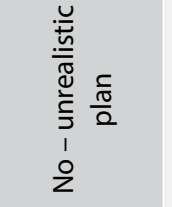 & $\stackrel{\check{\nu}}{\check{\nu}}$ & $\stackrel{\check{\beth}}{\check{\nu}}$ & zo & 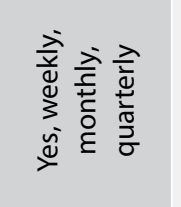 & $\stackrel{\check{\nu}}{\check{\nu}}$ & 苍 & $\stackrel{0}{2}$ \\
\hline 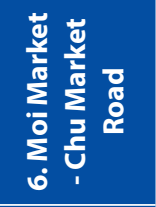 & 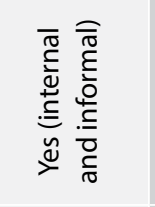 & 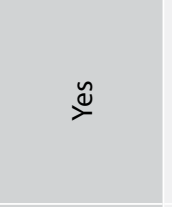 & $\stackrel{\check{\nu}}{\nearrow}$ & $\stackrel{\tilde{\nu}}{\check{\nu}}$ & 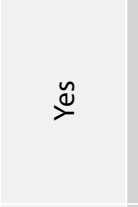 & 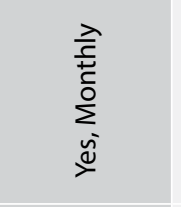 & $\stackrel{\pi}{z}$ & $\stackrel{\pi}{z}$ & $\stackrel{\pi}{z}$ \\
\hline 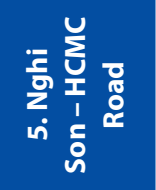 & 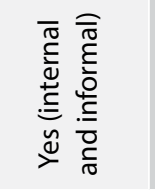 & $\overbrace{}^{\check{~}}$ & $\stackrel{\check{\Perp}}{\check{\nu}}$ & $\stackrel{\check{\varpi}}{\check{\nu}}$ & $\stackrel{\pi}{z}$ & 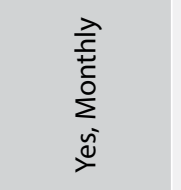 & 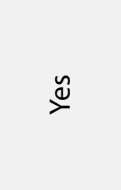 & 㟥芯 & $\stackrel{\circ}{z}$ \\
\hline 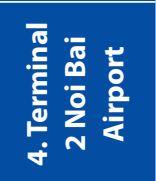 & 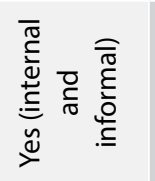 & $\stackrel{\check{\nu}}{\check{\nu}}$ & $\stackrel{\check{D}}{=}$ & $\stackrel{\check{\nu}}{\check{\nu}}$ & $\stackrel{\check{\nu}}{\check{\nu}}$ & 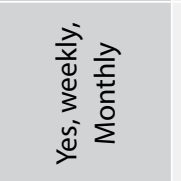 & $\stackrel{\check{\nu}}{\check{\nu}}$ & 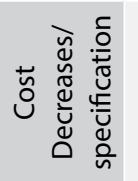 & 울 \\
\hline 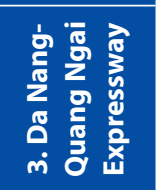 & 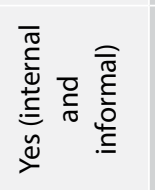 & $\stackrel{\check{\nu}}{\check{\Perp}}$ & $\stackrel{\Xi}{\nu}$ & $\stackrel{*}{\stackrel{*}{2}}$ & $\stackrel{\varrho}{\check{\nu}}$ & 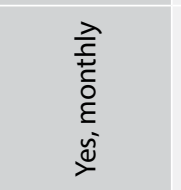 & 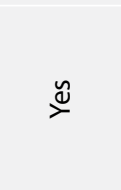 & 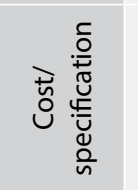 & 운 \\
\hline 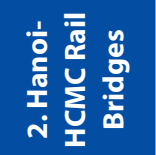 & 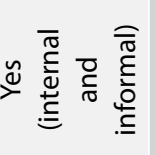 & $\stackrel{\check{\Perp}}{\check{\nu}}$ & $\stackrel{\tilde{\nu}}{\varnothing}$ & $\stackrel{\check{\varpi}}{\check{\nu}}$ & zo & 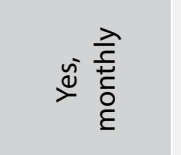 & $\stackrel{\check{\nu}}{\check{\nu}}$ & 苍 & $\stackrel{2}{2}$ \\
\hline 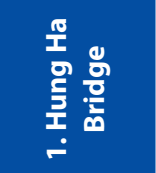 & 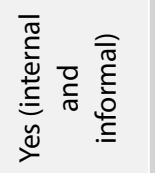 & $\stackrel{\check{\varpi}}{\rightleftharpoons}$ & 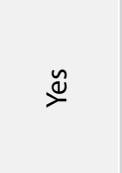 & $\stackrel{\check{\Perp}}{\rightleftharpoons}$ & $\stackrel{\widetilde{\nu}}{\check{\nu}}$ & 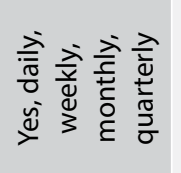 & 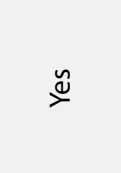 & 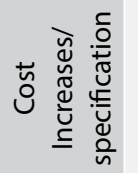 & $\stackrel{0}{z}$ \\
\hline & 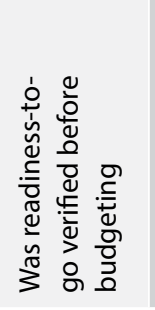 & 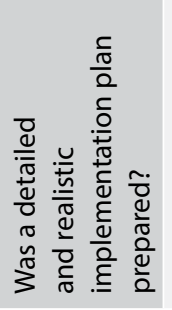 & 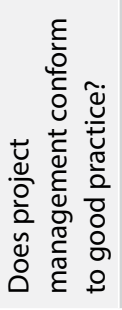 & 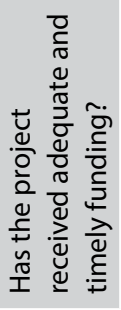 & 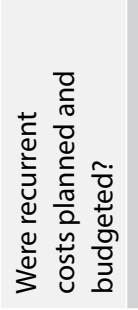 & 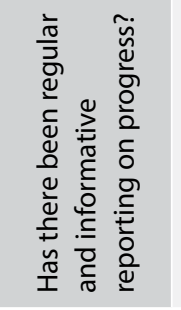 & 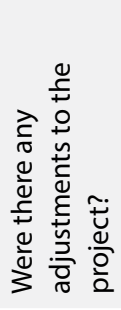 & 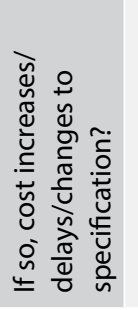 & 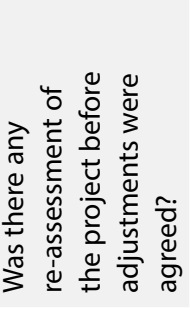 \\
\hline
\end{tabular}




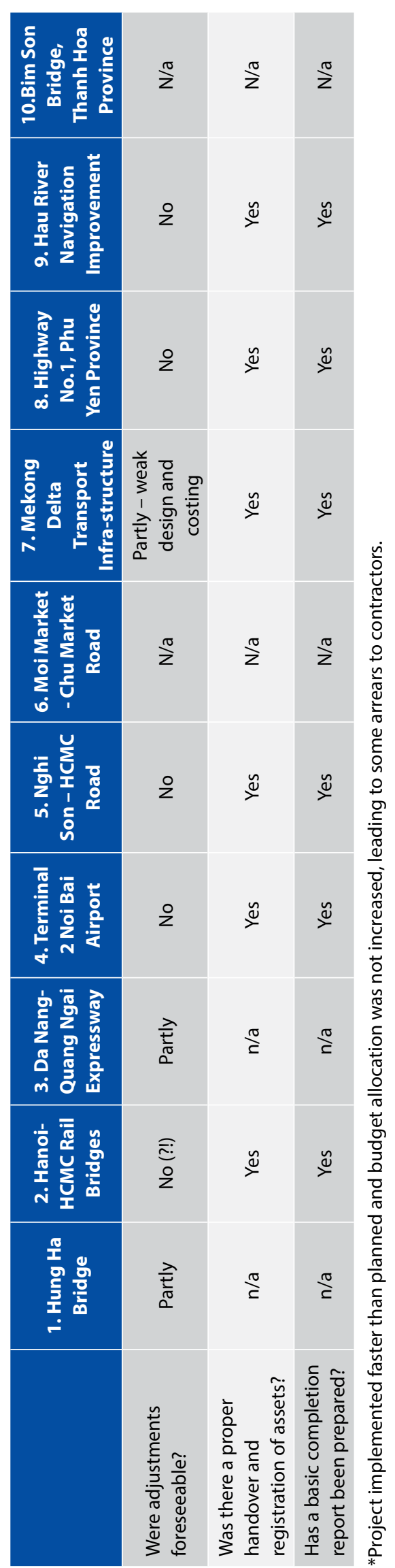









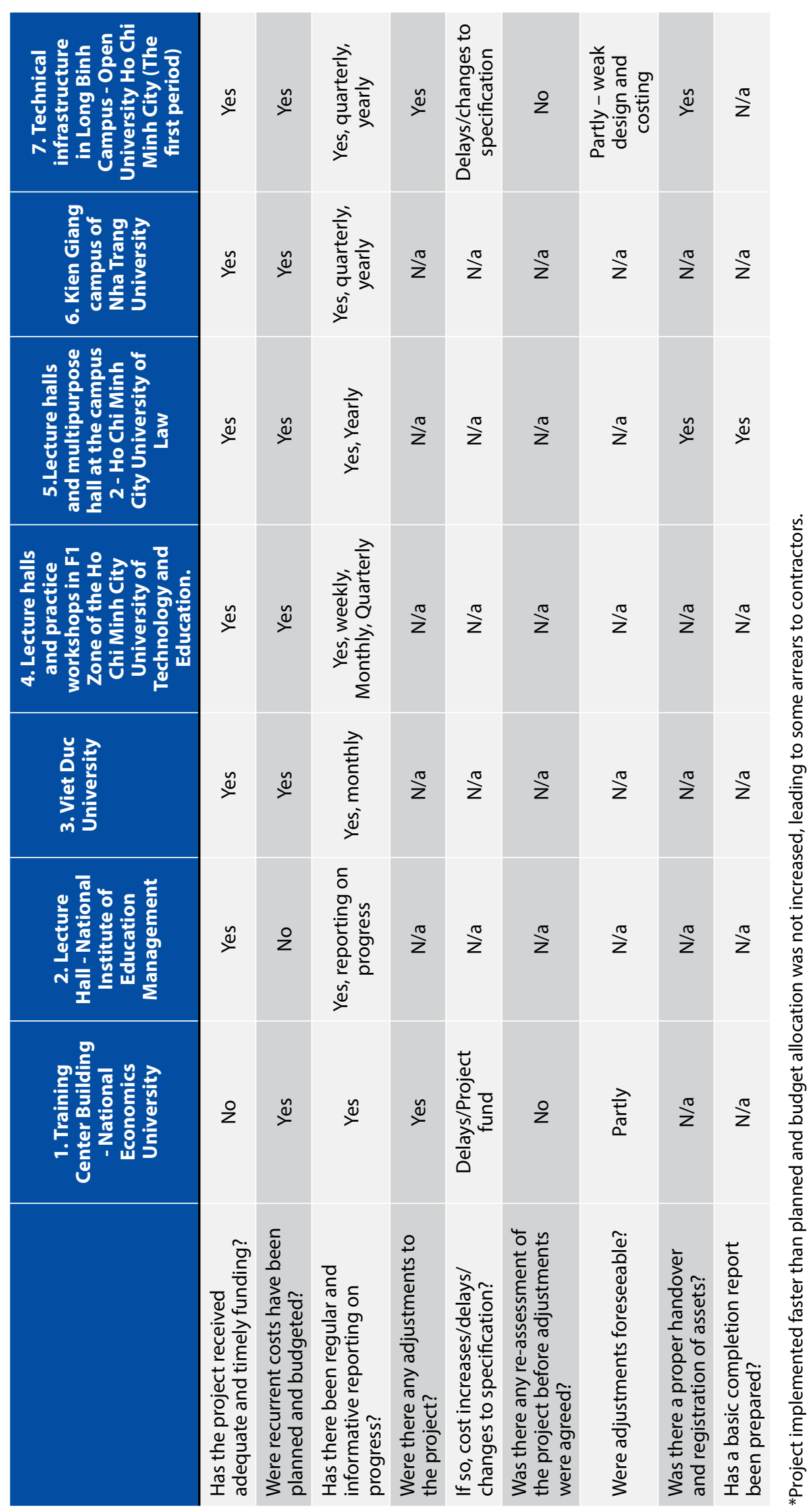


Publishing licence No: 2236-2018/CXBIPH/16-95/TN and 1067/QĐ-NXBTN issued on 20 August 2018 Designed by Golden Sky Co.,Ltd. | www.goldenskyvn.com 

With support from:

\section{Australian}

Aid

8 Dao Tan Street, Ba Dinh District, Hanoi, Vietnam Telephone: +842437740100

Facsimile: +84 2437740111

Website: www.dfat.gov.au

\section{(4) THE WORLD BANK \\ IBRD•IDA | WORLD BANKGROUP}

8th Floor, 63 Ly Thai To Street, Hoan Kiem District, Hanoi, Vietnam

Telephone: +84 2439346600

Facsimile: +84 2439346597

Website: www.worldbank.org/en/country/vietnam

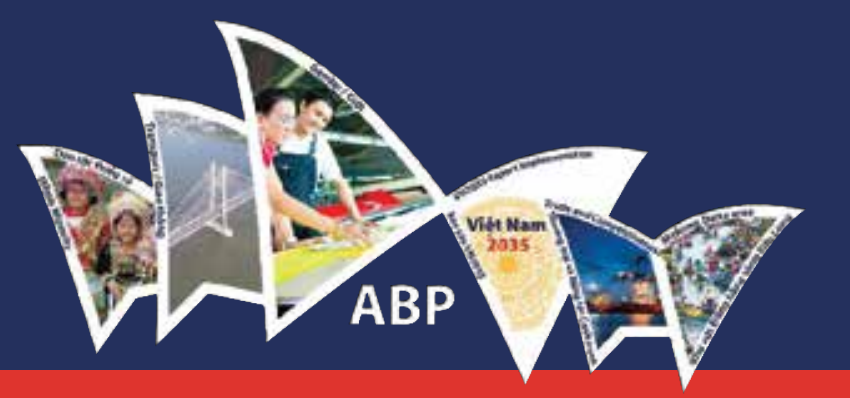

